\title{
WEAR MECHANISM AND WEAR PREVENTION IN COAL-FUELED DIESEL ENGINES
}

Task VII: Extended Wear Testing

Toplcel Report

By

J. F. Wakenell

S. G. Fritz

J. A. Schwalb

July 1991

Work Performed Under Contract Ho, AC21-89MC26044

For

U.S. Departinem of Energy Morgantown Energy Technology Celnter

Morgantown, West Virginia

By

Southwest Research Insthute

San Antonlo, Texas 


\section{DISCLAIMER}

This report was prepared as an account of work sponsored by an agency of the United States Government. Neither the United States Government nor any agency thereof, nor any of their employees, makes any warranty, express or implied, or assumes any legal liability or responsibility for the accuracy, completeness, or usefulness of any information, apparatus, product, or process disclosed, or represents that its use would not infringe privately owned rights. Reference herein to any specific commercial product, process, or service by isade name, trademark, manufacturer, or otherwise does not necessarily constitute or imply its enidoisement, recommendation, or favoring by the United States Government or any agency thereof. The views and opinions of authors ex. pressed herein do not necessarily state or reflect those if the United States Government or any agency thereof.

This report has been reproduced directly fron the best available copy.

Available to DOE and DOE contractors from the Office of Scientific and Technical Information, P.O. Box 62, Oak Ridge, TN 37831 ; prices available from (615)576-8401, FTS 626-8401.

Available to the public from the National Tichnical Information Service, U. S. Department of Commerce, 5285 Port Royal Rd., Springfield, VA 22161. 
Wear Mechanism and Wear Prevention in Coal-Fueled Diesel Engines

Task VII: Extended Wear Testing

\author{
Topical Report
}

J.F.Wakenell

S.G.Fritz

J.A.Schwalb

Work Performed Under Contract No.: DE-AC21-89MC26044

\title{
For \\ U.S. Department of Energy
}

Office of Fossil Energy

Morgantown Energy Technology Center

P.0. Box 880

Morgantown, West Virginia 26507-0880

\author{
By \\ Southwest Research Institute \\ Engine, Fuel, and Vehicle Research Division \\ 6220 Culebra Road \\ San Antonio, Texas 78228-0510
}

July 1991 


\section{TABLE OF CONTENTS}

\section{$\underline{\text { Page }}$}

I. INTRODUCTION $\ldots \ldots \ldots \ldots \ldots \ldots \ldots \ldots \ldots \ldots$

II. EXPERIMENTAL APPARATUS AND PROCEDURE $\ldots \ldots \ldots \ldots 1$

A. Engine Description $\ldots \ldots \ldots \ldots \ldots \ldots \ldots \ldots \ldots \ldots$

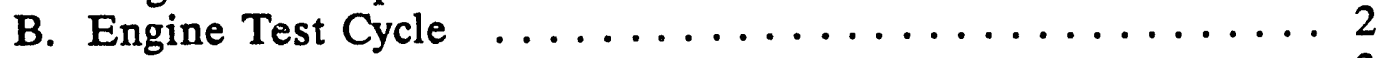

C. Engine Instrumentation $\ldots \ldots \ldots \ldots \ldots \ldots \ldots \ldots$

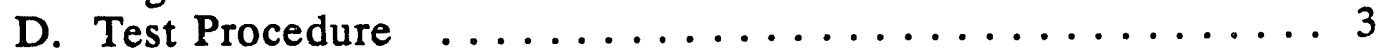

III. FUELS $\ldots \ldots \ldots \ldots \ldots \ldots \ldots \ldots \ldots \ldots \ldots \ldots \ldots \ldots$

IV. EXPERIMENTAL RESULTS $\ldots \ldots \ldots \ldots \ldots \ldots \ldots \ldots$

A. Performance Tests $\ldots \ldots \ldots \ldots \ldots \ldots \ldots \ldots \ldots$

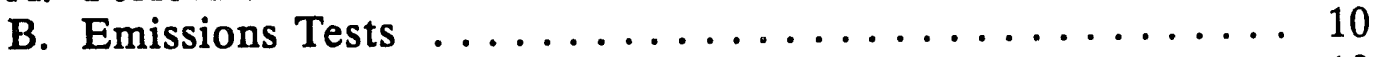

1. Gaseous Emission Measurement . . . . . . . . 10

2. Particulate Emission Measurement $\ldots \ldots \ldots \ldots \ldots 12$

3. Emission Test Results ................ 12

C. Wear Results $\ldots \ldots \ldots \ldots \ldots \ldots \ldots \ldots \ldots \ldots \ldots$

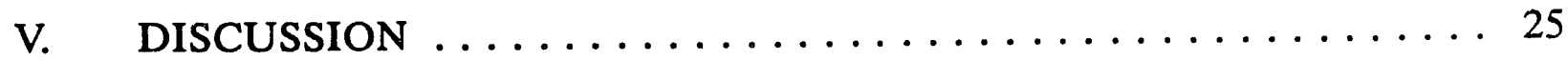

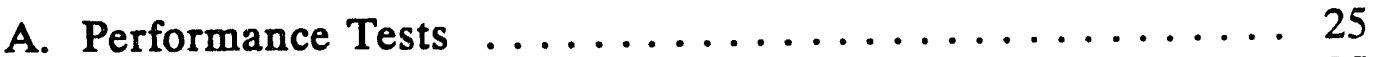

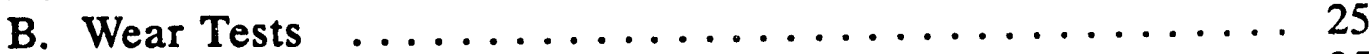

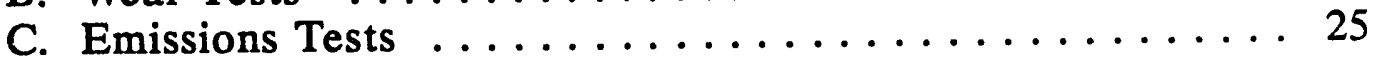

VI. CONCLUSIONS $\ldots \ldots \ldots \ldots \ldots \ldots \ldots \ldots \ldots \ldots \ldots \ldots$

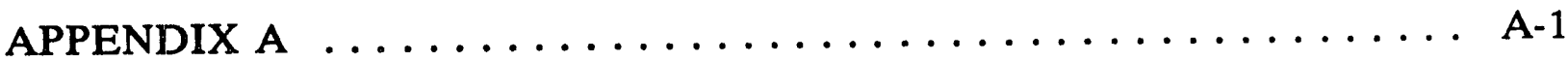

APPENDIX B $\ldots \ldots \ldots \ldots \ldots \ldots \ldots \ldots \ldots \ldots \ldots \ldots \ldots \ldots \ldots \ldots$

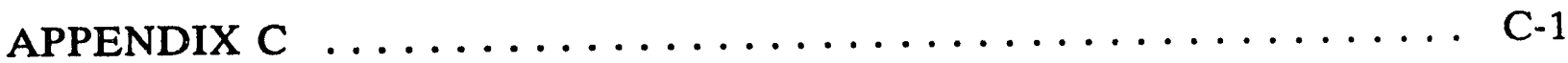

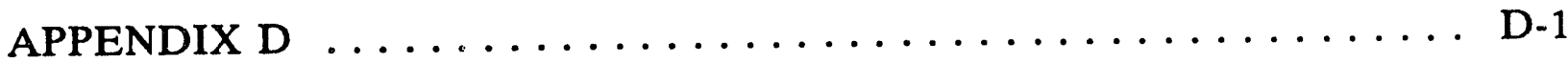

APPENDIX E $\ldots \ldots \ldots \ldots \ldots \ldots \ldots \ldots \ldots \ldots \ldots \ldots \ldots \ldots \ldots \ldots \ldots \ldots$

APPENDIX F $\ldots \ldots \ldots \ldots \ldots \ldots \ldots \ldots \ldots \ldots \ldots \ldots \ldots \ldots \ldots \ldots \ldots \ldots$ 


\section{LIST OF ILLUSTRATIONS}

Figure

Page

1 BHP Versus Notch Position $\ldots \ldots \ldots \ldots \ldots \ldots \ldots \ldots$

2 BMEP Versus Notch Position $\ldots \ldots \ldots \ldots \ldots \ldots \ldots \ldots$

3 Fuel Rate Versus Notch Position $\ldots \ldots \ldots \ldots \ldots \ldots \ldots$

4 T.E. Versus Notch Position $\ldots \ldots \ldots \ldots \ldots \ldots \ldots$

$5 \quad$ BSFC Versus Notch Position $\ldots \ldots \ldots \ldots \ldots \ldots \ldots \ldots$

6 EMD 2-567 Engine Corrected $\mathrm{NO}_{\mathrm{x}}$ Mass Emission Rate at

Each Notch Position, Before and After a 500-Hour

Durability Test, on Both Test Fuels ................. 14

7 EMD 2-567 Engine Caroon Monoxide Mass Emission Rate at

Each Notch Position, Before and After a 500-Hour

Durability Test, on Both Test Fuels ................ 15

8 EMD 2-567 Engine Hydrocarbon Mass Emission Rate at

Each Notch Position, Before and After a 500-Hour

Durability Test, on Both Test Fuels ................ 16

9 EMD 2-567 Engine Average Particulate Mass Emission

Rate at Each Notch Position, Before and After a 500-Hour

Durability Test, on Both Fuels

$10 \mathrm{SO}_{2}$ EMD 2-567 Engine $\mathrm{SO}_{2}$ Emission Rate at Each Notch Position, Before and After a 500-Hour Durability Test,

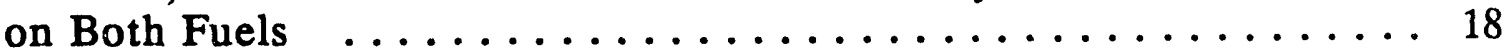

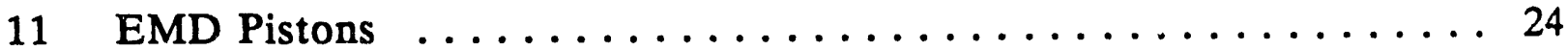




\section{LIST OF TABLES}

Table

Page

1 Engine Specifications $\ldots \ldots \ldots \ldots \ldots \ldots \ldots \ldots \ldots \ldots \ldots$

2 Engine Speed and Fuel Flowrate Combinations $\ldots \ldots \ldots \ldots 2$

3 Properties of the Engine Test Fuels $\ldots \ldots \ldots \ldots \ldots \ldots \ldots \ldots$

4 Summary of Emissions Data for the EMD 2-567

Locomotive Engine .................... 19-22

5 Weighted Composite Brake Specific Emissions Summary for the EMD 2-567 Locomotive Engine . . . . . . . . . . . . 23 


\section{INTRODUCTION}

Over the past several years, interest has arisen in the development of coalfired diesel engines for the purpose of efficiently utilizing the extensive coal reserves in the United States, and therefore reducirig dependence on foreign oil. One process which is being considered for use in producing clean coal fuel products involves mild gasification. This process produces by-products which can be further refined and, when blended with neat diesel fuel, used as an engine fuel. The purpose of this task was to test a blend of this coal liquid and diesel fuel (referred to as coal-lite) in an engine, and determine if any detrimental results were observed. This was done by performing a back-to-back performance and emission test of neat diesel fuel and the coal-lite fuel, followed by a 500-hour test of the coal-lite fuel, and completed by a back-to-back performance and emission test of the coal-lite fuel and neat diesel fuel.

\section{EXPERIMENTAL APPAKATUS AND PROCEDURE}

\section{A. Engine Description}

The engine chosen as the test bed in this experiment was the two-cylinder version of the Electro-Motive Division of General Motors (EMD) 567 locomotive engine. This engine was chosen specifically because one of the markets for this fuel is the railroad industry. The two-cylinder engine was used, quite simply, because of the lower operating costs and fuel consumption and lower repair costs if detrimental results were observed. The EMD two-cylinder 567 engine was a limited production research engine built by EMD and designed specifically for research use. The engine is a two-stroke cycle, blower-scavenged unit with a displacement of 567 cubic inches per cylinder that produces 215 brake horsepower at $835 \mathrm{rpm}$. Details of the engine are presented in Table 1 .

\begin{tabular}{||l|c|}
\hline \multicolumn{2}{|c|}{ Table 1. Engine Specifications } \\
\hline & EMD 2-567B \\
\hline Number of Cylinders & 2 \\
\hline Displacement (cu. in./cyl.) & 567 \\
\hline Bore and Stroke (in.) & $8.5 \times 10$ \\
\hline Rated Speed (rpm) & 835 \\
\hline Rated Brake Horsepower & 215 \\
\hline Compression Ratio & 16.1 \\
\hline Cycle & 2 \\
\hline Injection System & Unit Injector \\
\hline
\end{tabular}


The 567 series engines were produced and used by the railroad industry in 6, 8-, 12-, and 16-cylinder configurations. The engine is loaded by a DC generator and the power is absorbed by a resistive load grid, water cooled for heat dissipation.

\section{B. Engine Test Cycle}

The engine was operated at conditions that simulated the notch operation of a locomotive. Locomotive engines operate only at specified speed/power combinations defined by throttle positions or "notches." Since the power output and speed are constant at each notch position, the fuel consumption rate is also constant and can be defined for each position. EMD engines in locomotive service operate at eight power producing notches. There are also idle and, in some locomotives, low idle operating positions. To facilitate fuel testing, the positions were redefined in terms of speed and load combinations (see Table 2).

\begin{tabular}{|c|c|c|c|}
\hline Notch Position & $\begin{array}{l}\text { Engine Speed } \\
\text { (RPM) }\end{array}$ & $\begin{array}{c}\text { Fuel Flowrate } \\
(\mathrm{kg} / \mathrm{hr})\end{array}$ & Typical BHP \\
\hline $8^{*}$ & 835 & 40.8 & 207 \\
\hline 7 & 755 & 34.0 & 178 \\
\hline 6 & 675 & 27.2 & 149 \\
\hline 5 & 585 & 20.4 & 114 \\
\hline 4 & 515 & 15.0 & 77 \\
\hline 3 & 425 & 9.5 & 47 \\
\hline 2 & 345 & 5.4 & 20 \\
\hline 1 & 285 & 3.6 & 8 \\
\hline Idle & 285 & 2.5 & 0 \\
\hline
\end{tabular}

Fuel rate for the two-cylinder EMD 567 research engine was taken as $1 / 8$ the typical values for the 16 -cylinder $567 \mathrm{C}$ with $16: 1$ compression ratio pistons. Unlike revenue service locomotive engines, the two-cylinder engine is equipped with a 
pneumatic governor that permits infinite variations of both speed and load. However, during testing the engine was operated only at the speeds and fuel consumption rates corresponding to the notch schedule presented in Table 2:

Performance data was corrected to standard ambient conditions in all cases. Factors defined by EMD were applied to correct performance to $60^{\circ} \mathrm{F}$ and 29.9 in$\mathrm{Hg}$. Engine test data have shown the engine manufacturers recommended factors to provide the best correlation for correction.

\section{Engine Instrumentation}

The test engine was instrumented to monitor engine speed, power output and pressures and temperatures throughout the lubricating, cooling, intake and exhaust systems. Smoke density was measured using the Bosch method. A Micro-Motion mass flowmeter provided a continuous mass fuel flow rate measurement.

Engine instrumentation was connected to a low-speed data acquisition system. In addition to monitoring pressures and temperatures, the computer system also monitored alternator voltage and amperage output, the Micro-Motion voltage signal and engine speed. A computer averaging routine was employed for all performance measurements. During the performance tests, signals were recorded once every five seconds for approximately 20 minutes to provide a total of 250 data sampling readings. The 250 readings were averaged and the power output, thermal efficiency, mean effective pressure, and brake specific fuel consumption were calculated based on these averages. This procedure accounted for periodic oscillations in power output and fuel consumption rate and resulted in an accurate fuel consumption measurement.

\section{Test Procedure}

The study began with a tear-down and reassembly of the engine power packs (pistons, rings, and liners) for baseline measurement. Ring and liner measurements were performed using standard measurement instruments. Two new fuel injectors were "pop tested" for conformation of integrity and installed in the engine. Next, a nine hour baseline neat diesel fuel performance, economy, and emission test at all eight throttle notches (and idle) was performed.

Next, in order to evaluate the affects of the test (coal-lite) fuel, the engine was purged with the test fuel and a nine hour performance, economy, and emission test run using the test fuel. Data was recorded at each notch position. Upon completion of the performance test, the 500 hour durability test was performed. This test consists of 250 two-hour cycles at Notch 8,5 , and idle. During the durability test, performance data was recorded at Notch eight only. Emissions measurements were not taken during the durability test. Following the 500 hour test, another nine hour 
performance, economy, and emission test using the test fuel was conducted in order to assess any performance loss as a result of engine wear. These results were compared to the pre-500 hour test fuel run.

The final engine test was performed using neat diesel fuel for a comparison to the baseline test.

Upon completion of the engine tests the engine power packs were removed and disassembled for inspection and measurement. Photographs were taken to visually record engine wear and carbon deposition. Engine wear was assessed based on a comparison to the initial measurement and visual inspection. The fuel injectors were again "pop tested" to check for injector dribbling. After which, the injectors were disassembled and inspected and measured.

\section{FUELS}

The fuels used for this evaluation were neat diesel fuel and the coal-lite liquid. The neat diesel fuel was a commercially available No. 2 diesel. Chemical analysis of the fuels are presented in Table 3.

\section{EXPERIMENTAL RESULTS}

\section{A. Performance Tests}

The performance test results for all four performance tests are shown in Figures 1 through 5. Basically, there is very little difference between all the fuels. During the tests, horsepower was held constant as indicated in Figure 1. Therefore, there also should not be any differences in BMEP as shown in Figure 2. Because horsepower was held constant, any performance differences encountered as a result of the fuel differences would be detected in fuel consumption, BSFC, and possibly thermal efficiency.

Shown in Figure 3, is a plot of fuel consumption for each notch position. For the most part, fuel consumption was approximately the same for Notches 1 through 5. At the higher notches, fuel consumption varied. At Notch eight, the first coal-lite test fuel consumption was 4 percent greater than the first baseline. This increase in fuel consumption could be a function of two factors. Shown in Figure 4, thermal efficiency at Notch eight for the first coal-lite test was 3 percent less than the first baseline. This is a function of how efficiently the combusted fuel (heat) is converted into work, and partially accounts for the increase in fuel consumption. The other one percent difference is most likely a result of the 1 percent lower heating value of the fuel as shown in Table 3. The combined effect of a lower heat of combustion and reduced thermal efficiency will account for the increased fuel consumption. Notch eight was used as the example here, however the same conclusion can be drawn for the other notches where the difference was significant. 
Table 3. Properties of the Engine Test Fuels

\begin{tabular}{|c|c|c|c|}
\hline Property & Procodinte & Neat Na 2 Diend Pad & Conl Lite Teal \\
\hline Gravits. APL, GOP & D129: & & 298 \\
\hline Specific Grovits, 60\% & & & 8767 \\
\hline $\begin{array}{l}\text { Distillntion, }{ }^{\circ} \mathrm{P:} \\
\text { BBP/5t } \\
10 / 20 \\
30 / 40 \\
50 / 60 \\
70 / 80 \\
90 / 95 \\
\text { EPe } \\
\text { Recover, \% } \\
\text { Residue, \&5 }\end{array}$ & Des & $\begin{array}{c}34 / 394 \\
425 / 461 \\
490 / 501 \\
525 / 540 \\
560 / 578 \\
611 / 636 \\
660 \\
995 \\
05 \\
\end{array}$ & $\begin{array}{l}354 / 392 \\
425 / 456 \\
490 / 498 \\
518 / 534 \\
555 / 573 \\
619 / 633 \\
653\end{array}$ \\
\hline $\begin{array}{l}\text { Viscosits. } \\
\text { ast @ 104. }\left(40^{\circ} \mathrm{C}\right) \\
\text { SUS @ 104 }\end{array}$ & DMS & & 28 \\
\hline Flash Point, ${ }^{\circ} \mathbf{F}$ & D93 & & 154 \\
\hline 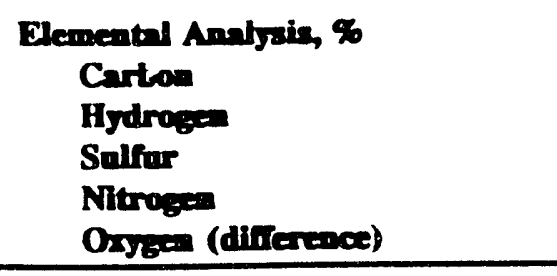 & $\begin{array}{c}\text { DB178 } \\
\text { CEC 240 } \\
\text { Xer } \\
\text { Cremilnomi } \\
\text { nescent }\end{array}$ & $\begin{array}{l}86.77 \\
12.80 \\
0.324 \\
0.13\end{array}$ & $\begin{array}{l}86.22 \\
12.01 \\
0.506 \\
0.136 \\
0.92\end{array}$ \\
\hline 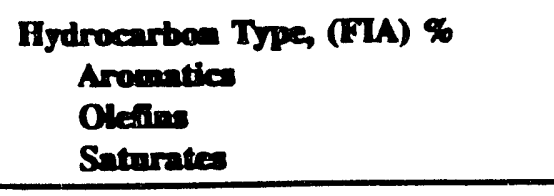 & D1319 & . & $\begin{array}{l}455 \\
12 \\
533\end{array}$ \\
\hline 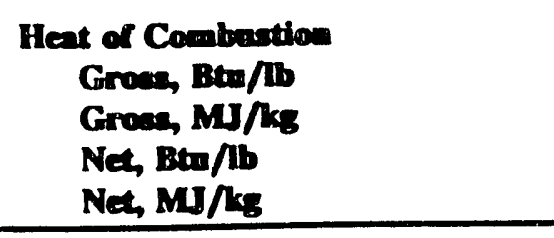 & D2NO & $\begin{array}{l}19415 \\
45.133 \\
18187 \\
42301\end{array}$ & $\begin{array}{l}19103 \\
4249 \\
17928 \\
41.702\end{array}$ \\
\hline Accelernted Stability, meg/100 ml & De274 & & 62 \\
\hline Carbon Residee, $10 \% 5$ Btons, wis & DEx & & 0.35 \\
\hline Particulates & $\operatorname{Dan} 76$ & & 126 \\
\hline 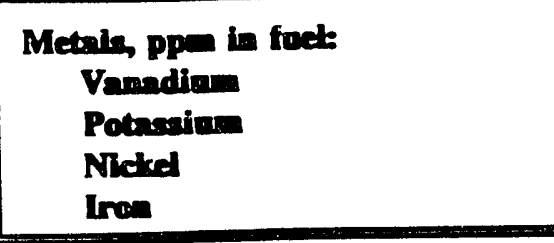 & ICP & & $\begin{array}{l}<1 \\
<25 \\
<1 \\
<1\end{array}$ \\
\hline
\end{tabular}

$a=$ Numbers preceded by a D are ASTM

d = Instrumental analysis
$b=I B P$ is the initial boiling point

- Indectively cooplad plasma $c=$ EP is the end point 


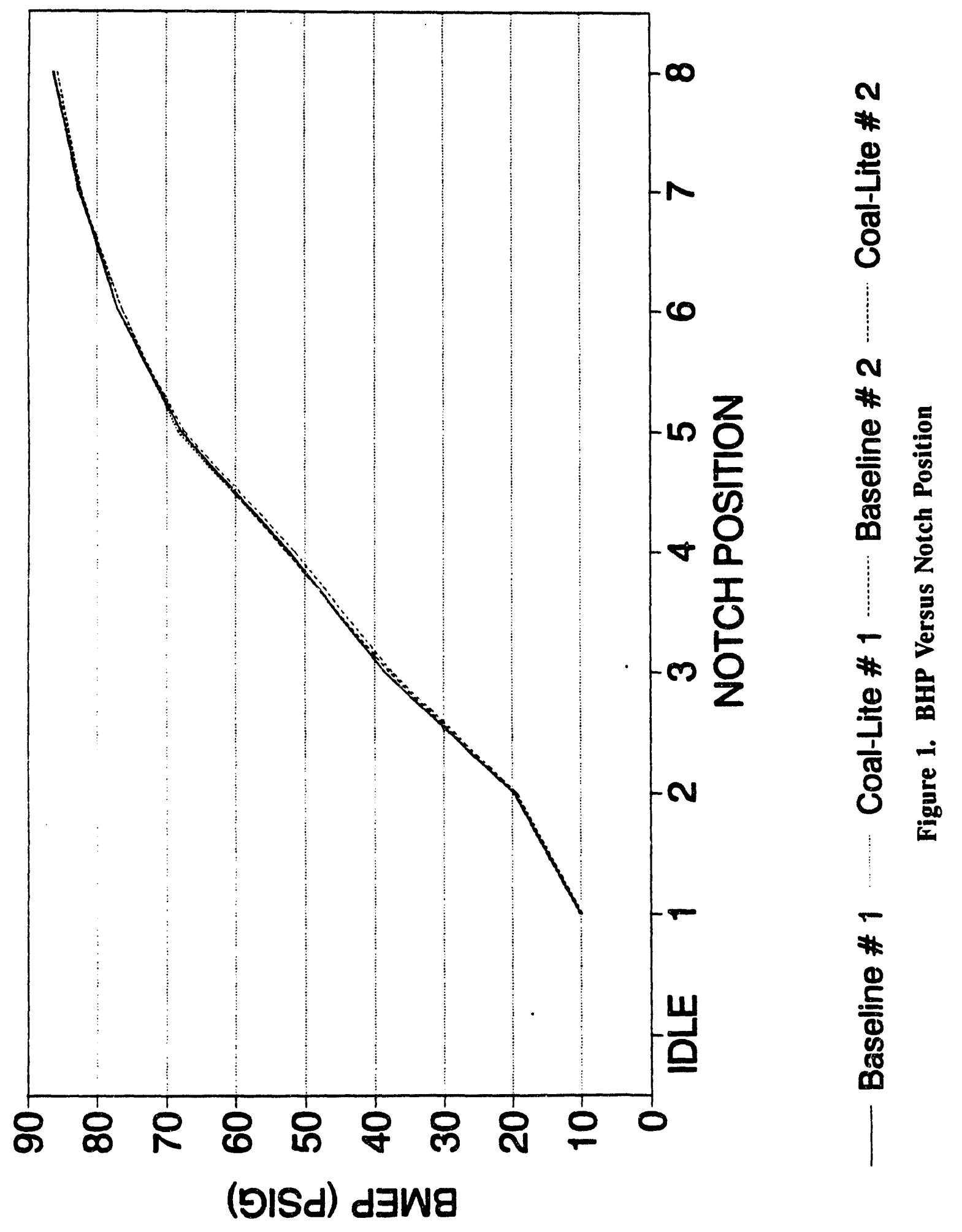




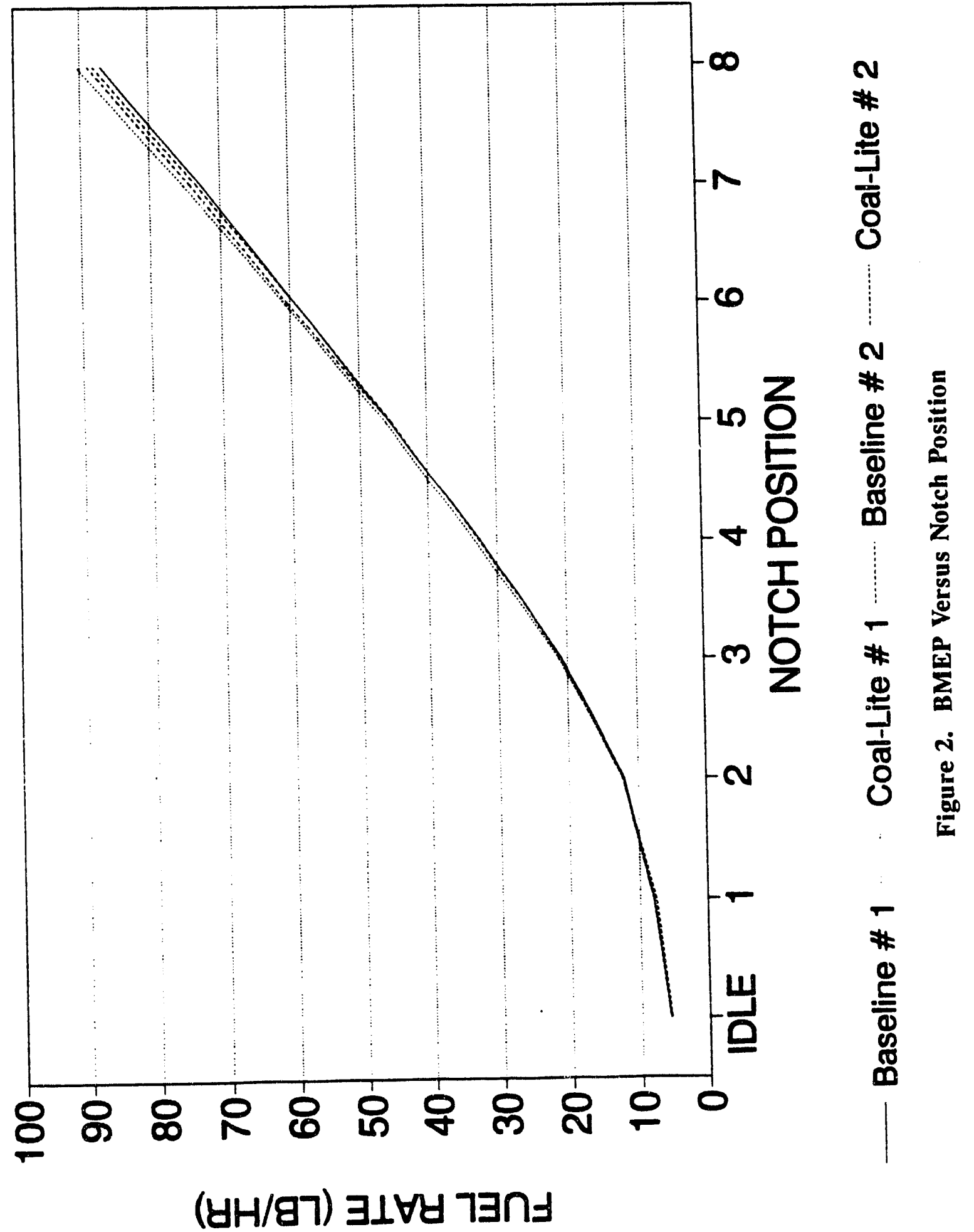




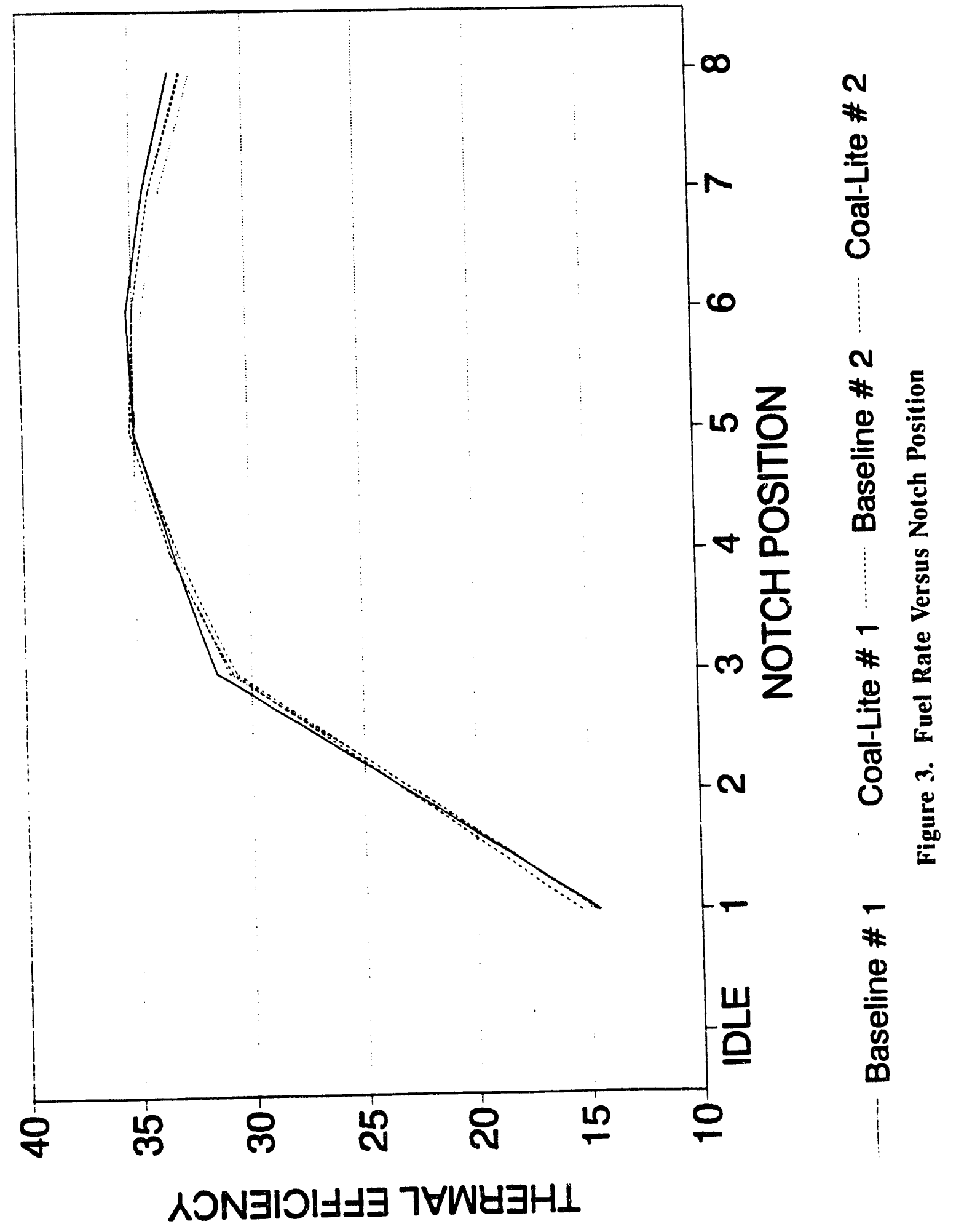




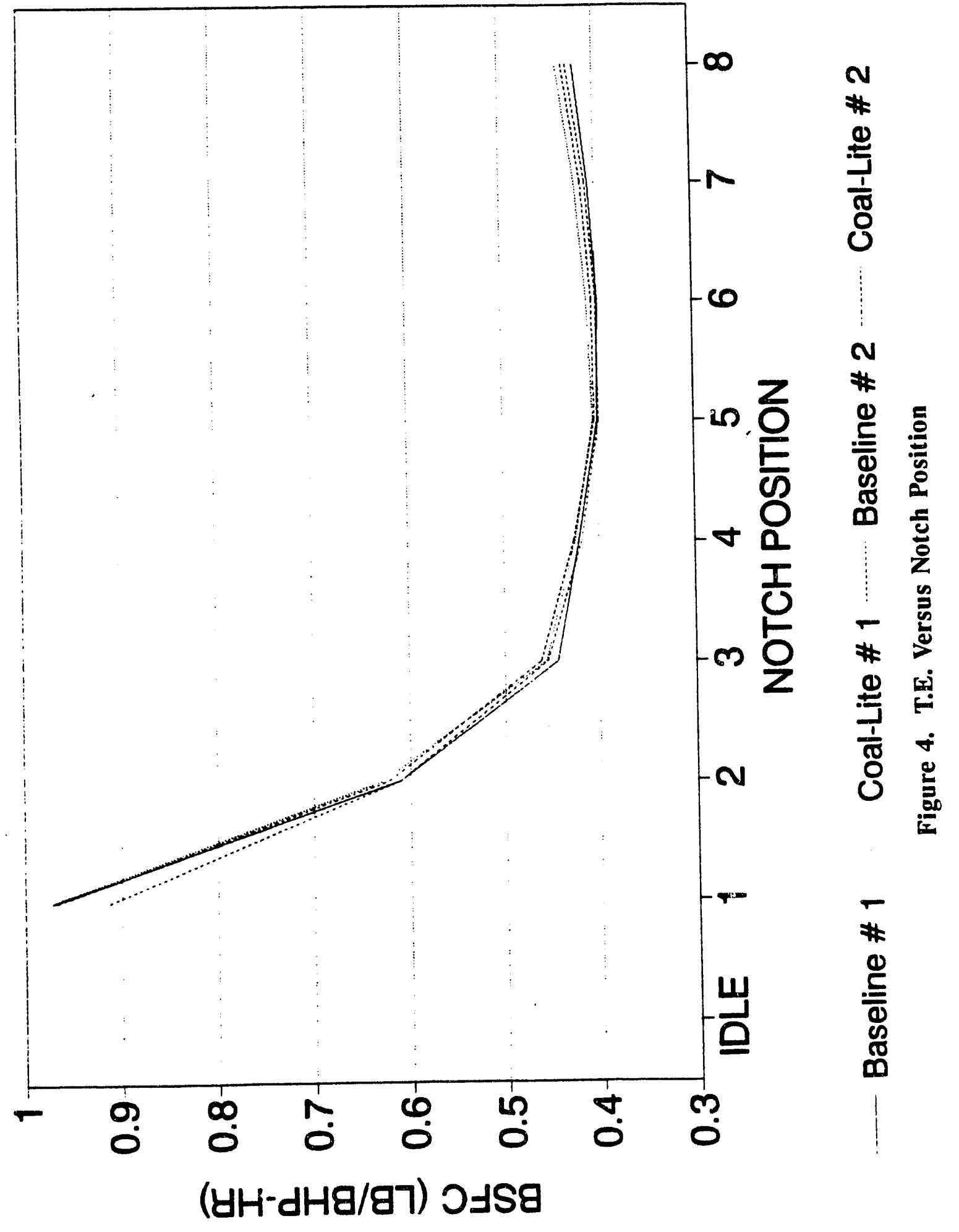


Also shown at Notch eight (Figure 3 ), is a roughly 2 percent increase in fuel consumption for the post 500-hour coal-lite test. The immediate question asked is why this differs from the first coal-lite test (4 percent increase). The explanation here is not quite as clear however, it is suspected that throughout the 500-hour test, the engine was continuing to seat in the rings and liners and that motoring friction was higher for the pre 500-hour tests. This would result in greater fuel consumption at the start of test for both fuels when compared to the end of test (500 hours) results, and in fact, that is what was observed.

Finally, shown in Figure 5, is a plot of BSFC verses notch position. BSFC is determined by dividing fuel rate by horsepower. Specific fuel consumption is a comparative parameter that describes how efficiently the engine converts fuel into work. BSFC comparisons are preferred to thermal efficiency because all parameters are measured in standard and accepted units: time, horsepower, and mass. In any case, both thermal efficiency and BSFC indicate how efficiently the engine is working and the trends shown in Figure 5 (BSFC) are the same as those shown in Figure 4 (thermal efficiency).

\section{B. Emissions Tests}

The Department of Emissions Research of Southwest Research Institute (SwRI) performed steady-state gaseous and particulate emission tests on the engine using both fuels. The tests were performed before and after the 500-hour engine durability test of the coal-lite fuel. This report section summarizes the emissions test procedure and gives a detailed listing of the emission results as well as a composite emissions factor comparison of the baseline diesel fuel and the coal-lite fuel.

\section{Gaseous Emission Measurement}

Gaseous emission measurements during each steady-state test condition were obtained by sampling raw exhaust following procedures detailed in 40 CFR Part 86, Subpart D. Exhaust gases were analyzed for unburned hydrocarbons (HC), carbon monoxide $(\mathrm{CO})$, oxides of nitrogen $\left(\mathrm{NO}_{\mathrm{x}}\right)$, carbon dioxide $\left(\mathrm{CO}_{2}\right)$, and oxygen $\left(\mathrm{O}_{2}\right)$. Hydrocarbons were measured by a heated flame ionization detector (HFID) unit built to specifications given in SAE Recommended Practice J215. Carbon monoxide and carbon dioxide were measured by a non-dispersive infrared (NDIR) analyzer in a system that conforms to SAE Recommended Practice J177a. Oxides of nitrogen were measured using a chemiluminescent analyzer.

Sulfur dioxide $\left(\mathrm{SO}_{2}\right)$ mass emission rates were also determined for the EMD 2-567B engine. The exhaust of $\mathrm{SO}_{2}$ in the diesel exhaust was measured as sulfate 


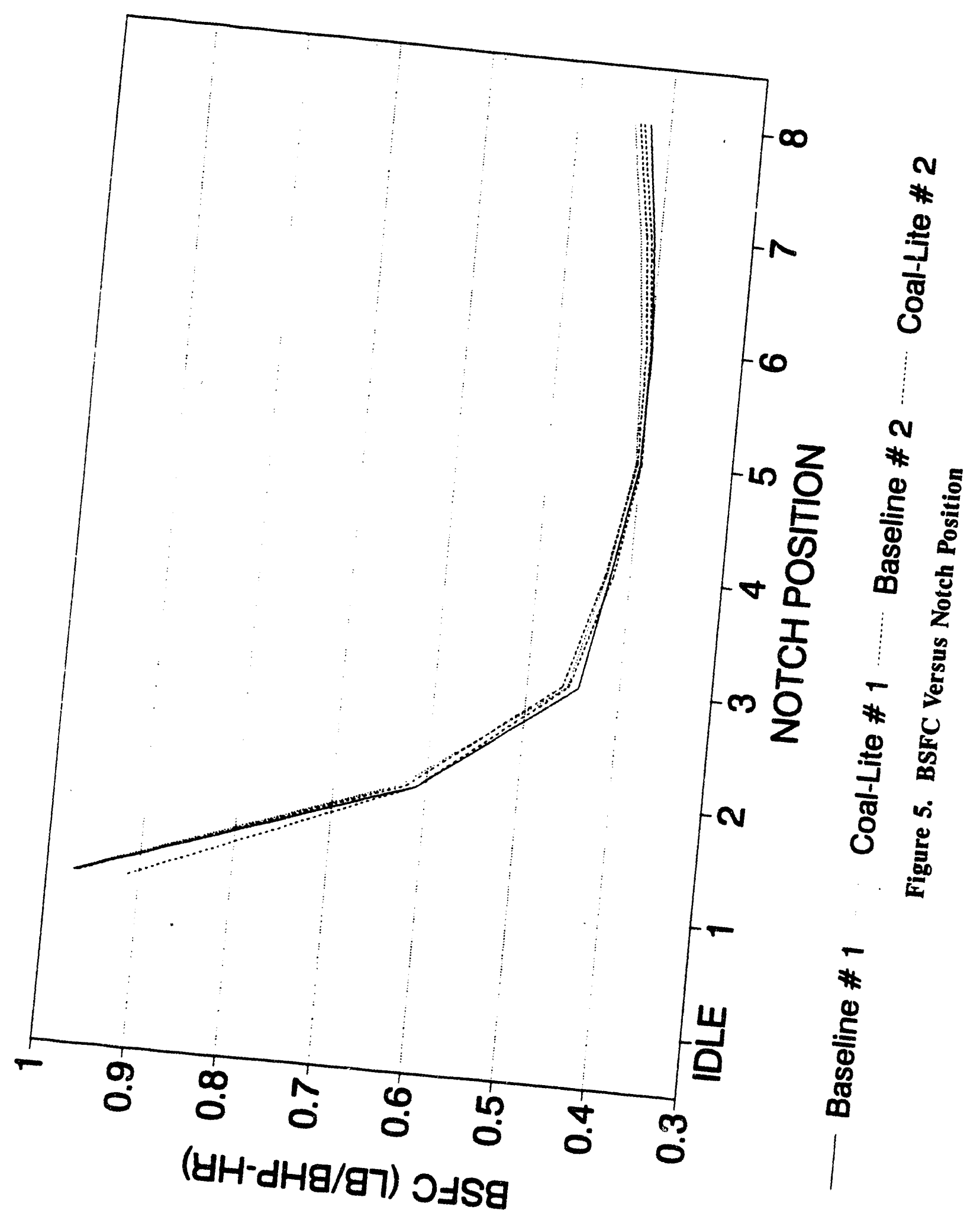


using an ion chromatograph following EPA procedures ${ }^{1} \cdot \mathrm{SO}_{2}$ exhaust samples were collected in two glass bubblers, each containing 3 percent hydrogen peroxide. The temperature of the absorbing solution was kept at $0^{\circ} \mathrm{C}$ by means of an ice water bath. The bubbled samples were analyzed on an ion chromatograph and compared to standards of known sulfate concentrations. The measured $\mathrm{SO}_{2}$ mass concentration in the exhaust were then compiled with the measured engine exhaust flow rates to compute the mass emission rate of $\mathrm{SO}_{2}$.

\section{Particulate Emission Measurement}

Particulate measurements during each steady-state test condition were obtained using a "splitter" dilution system. The splitter system splits off a portion of the total raw exhaust flow and mixes it with dilution air. Mixing occurs in a dilution tunnel prior to sampling the mixture for particulate. The stainless steel dilution tunnel used for this work was 20 centimeters ( 8 inches) in diameter and approximately 5 meters ( 15 feet long). Particulate mass samples were collected on dual 47 mm Pallflex T60A20 fluorocarbon-coated glass fiber filters (primary and backup). Dilution air and the split-off portion of the total exhaust were regulated such that the maximum temperature at the filter sample zone was $52^{\circ} \mathrm{C}\left(125^{\circ} \mathrm{F}\right)$. All filters were weighed in a temperature and humidity controlled chamber to ensure consistency of results.

\section{Emission Test Results}

Mass emission rates of $\mathrm{HC}, \mathrm{CO}, \mathrm{NO}_{x}$, particulates, and $\mathrm{SO}_{2}$ were computed using the raw gaseous emissions measurements, the particulate tunnel flow data along with the particulate filter weight gain, and the engine air flow, fuel flow, and power output data. For the test performed in this program, the power output (bhp) at each notch position was held constant and observations of the mass flow rate of the fuel were made.

Individual emission test results for each test point are given in Appendices AD. Included are the baseline pre 500-hour baseline test on diesel fuel (Appendix A), the pre 500-hour test on the coal-lite fuel (Appendix B), the post 500-hour test on the coal-lite fuel (Appendix C), and the final post 500-hour test on the baseline diesel (Appendix D).

A summary of the $\mathrm{NO}_{\mathrm{x}}$ mass emission rates are shown in Figure 6. Note that ambient air temperature and humidity are known to affect internal combustion engine exhaust emissions. $\mathrm{NO}_{\mathrm{x}}$ is especially sensitive to these factors, and as a result, NOx correction factors are commonly used to adjust observed $\mathrm{NO}_{\mathrm{x}}$ results to standard conditions. For this project, $\mathrm{SwRI}$ has reported $\mathrm{NO}_{\mathrm{x}}$ values corrected to:

1 "Analytical Procedures for Characterizing Unregulated Emissions from Vehicles Using Middle-Distillate Fuels" EPA Interim Report EPA-600/2-80-0068, 1980. 
1. $85^{\circ} \mathrm{F}$ engine intake air temperature,

2. 75 grains of water per pound of dry air humidity.

The engine intake air temperature correction factor and humidity correction factor vary with measured fuel/air ratio (i.e. engine load). These correction factors are used for EPA 13-mode steady-state testing and are referenced in 40 CFR, Subpart $\mathrm{D}$ 86.345-79. The $\mathrm{NO}_{\mathrm{x}}$ values shown in Figure 6 and Appendices A-D are the corrected values.

Figures $7,8,9$, and 10 give summaries of the measured mass emission rates at each notch position for carbon monoxide, hydrocarbons, particulates, and sulfur dioxide, respectively.

A duty-cycle based weighting procedure was used in determining composite brake specific emissions. The weighting factors used in determining composite brake-specific emissions are typical of a locomotive line-haul duty cycle, and are fairly representative of locomotive operations through out the U.S.; with approximately 10 percent of the time spent at rated conditions (Notch 8 ) and a significant amount of time (55 percent) at idle. The remainder of the time is spent distributed over the other throttle notch positions. Table 4 shows the un-weighted and weighted emission results, as well as the composite weighted brake specific exhaust emissions expressed in $\mathrm{g} / \mathrm{bhp}$-hr.

The composite brake specific emissions for the EMD 2-567B operating on both baseline ASTM 2D diesel and the coal-lite fuel are summarized in Table 5.

\section{Wear Results}

Engine wear was assessed based on visual inspection and physical wear measurements. Wear measurements were taken according to the standard EMD dimension sheets. Sheets 1 through 26 are presented in Appendix C. Generally, in 500 hours of operation, wear in this class of engine should be at the lower limits of measurable range. Measurable range using classical measurement tools is typically 0.001 " or greater. Therefore, wear reported less than 0.001 " can be considered beyond the range of measurement accuracy. However, wear greater than 0.001 " for this engine can be considered significant.

Of particular interest, when evaluating alternative fuels, is the ring and liner wear. These surfaces are normally the first to show signs of abnormal wear with undesirable fuels. As shown, on dimension sheets 13, 14, and 21, 22, the wear was 


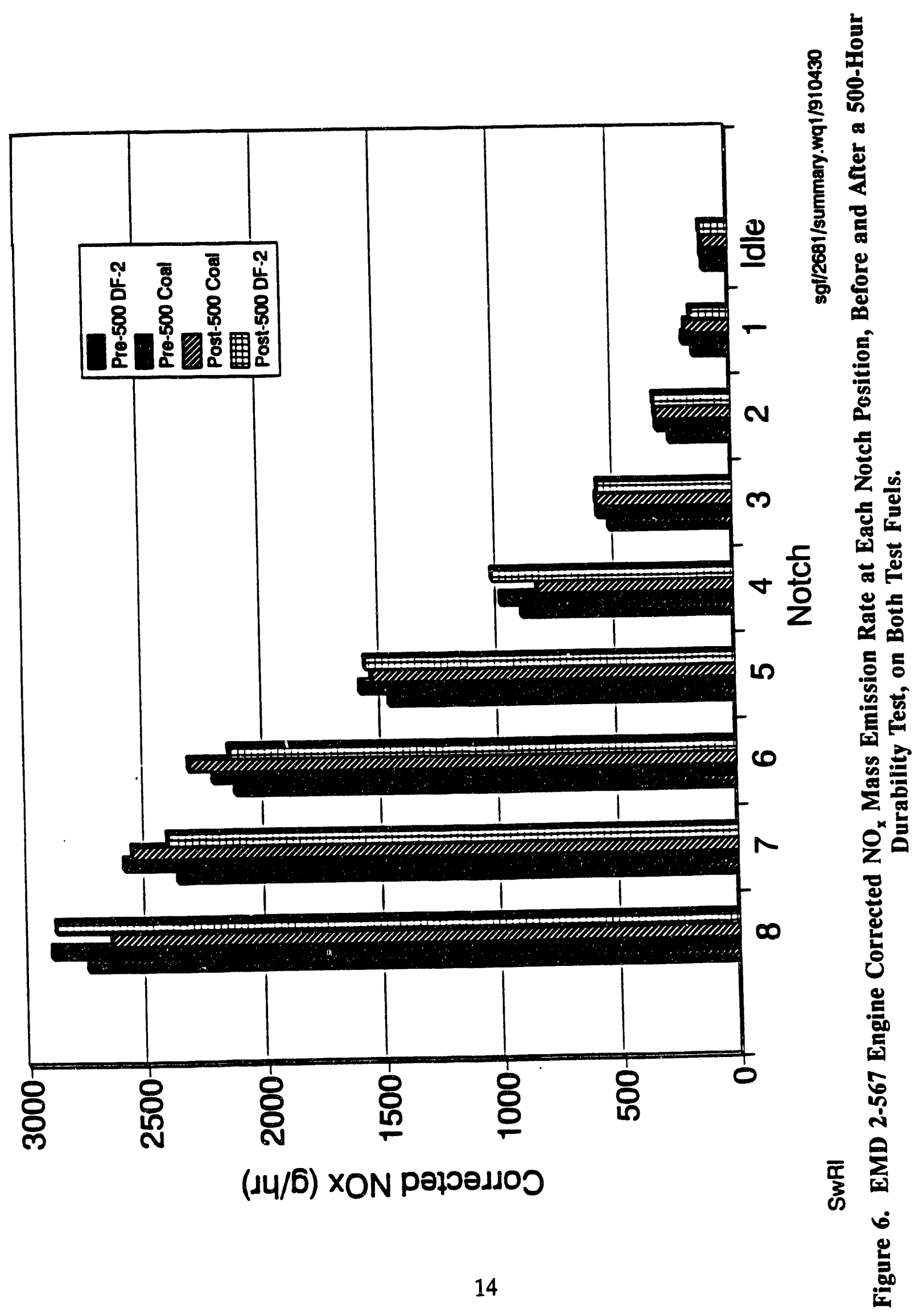




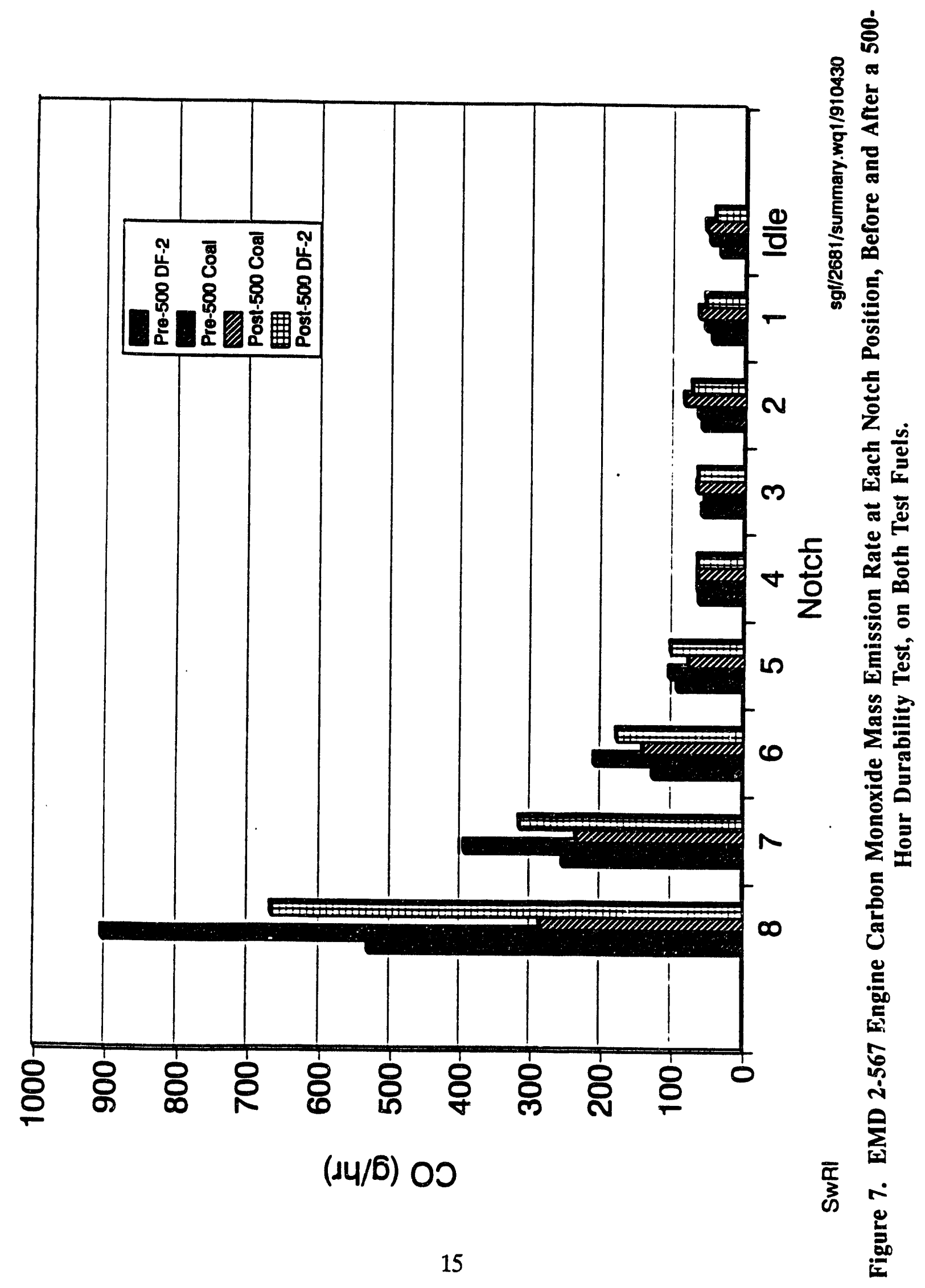




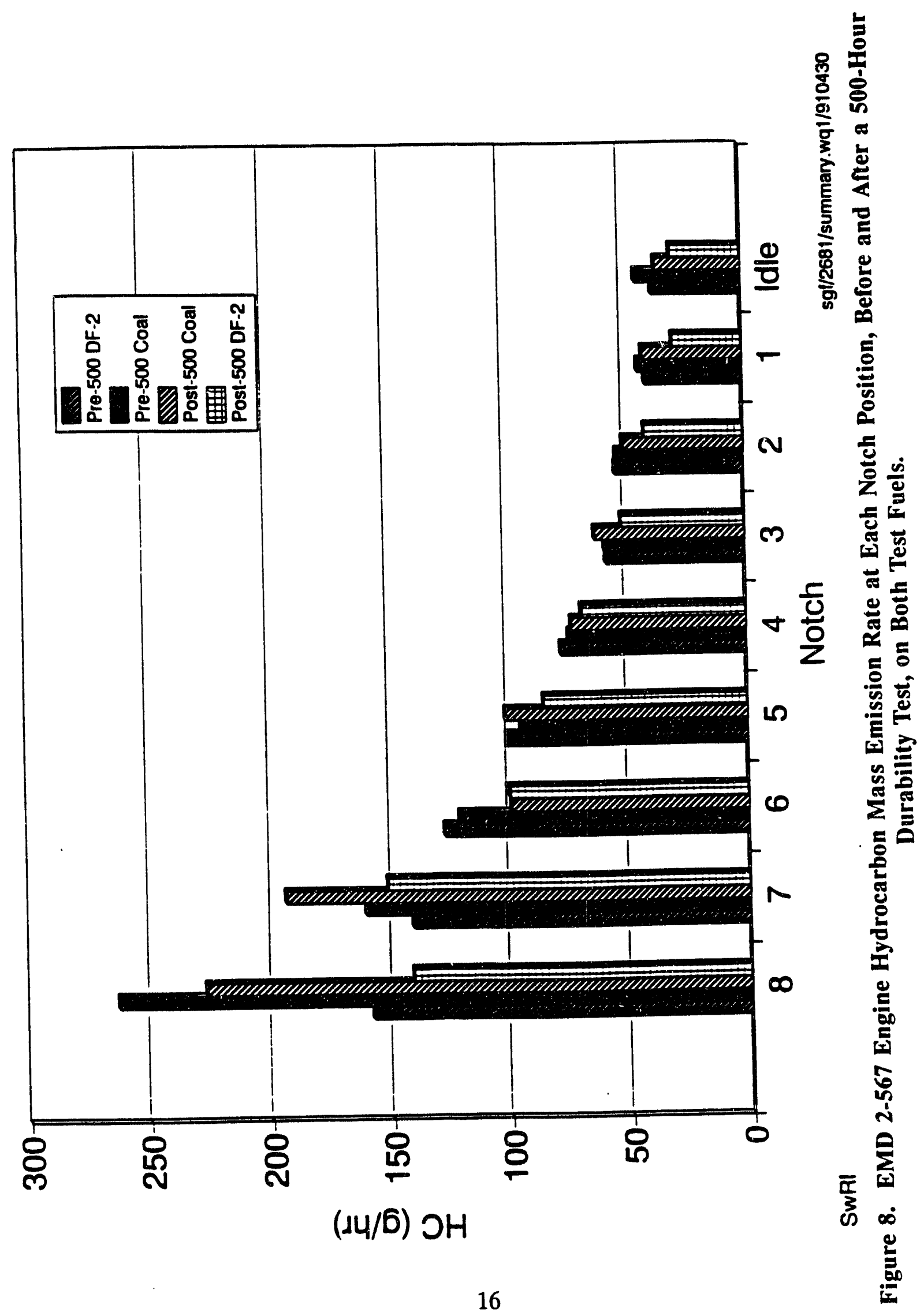




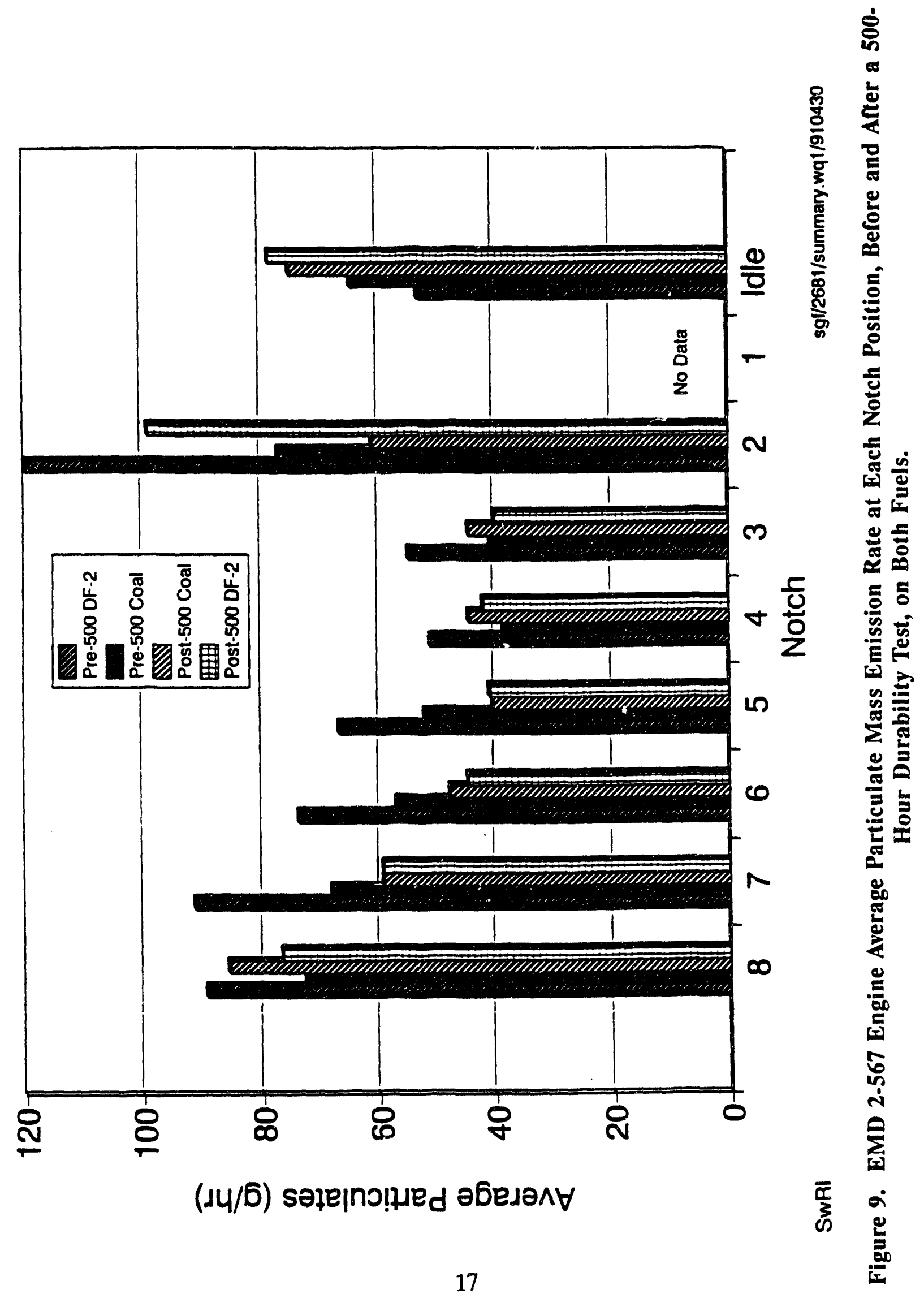




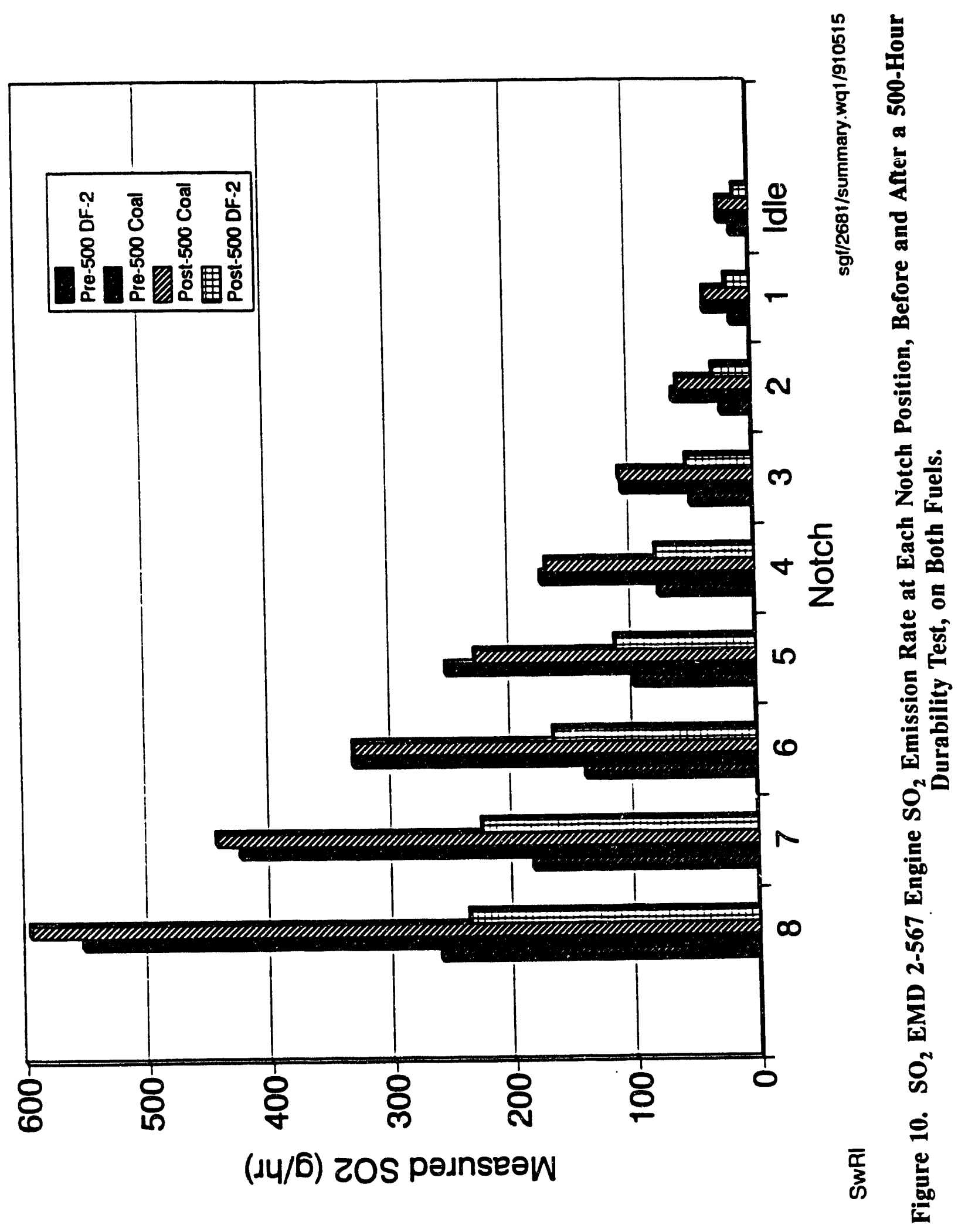




\begin{tabular}{|c|c|c|c|c|c|}
\hline & 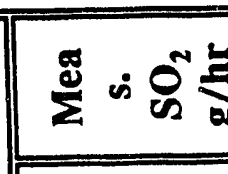 & $\therefore a-n$ & $m-1$ & $-\infty$ & \\
\hline & 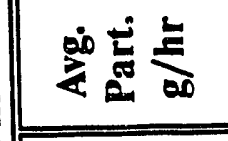 & $n=$ & & $+\pi$ & \\
\hline & हुํำ & 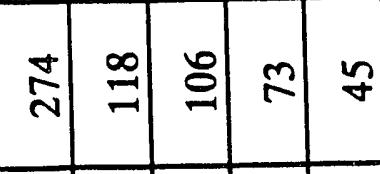 & $\approx=$ & $H=$ & $\equiv$ \\
\hline & 8 言 & $m=0 . m$ & $m$ & $\sim 2$ & \\
\hline & 品言 & $=7.0 .0$ & 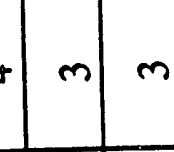 & $\sim \approx$ & $:$ \\
\hline 告 & 言 & $\bar{\nabla} a+0.0$ & $\sim-$ & 00 & 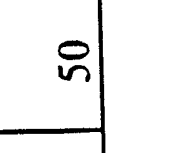 \\
\hline 音 & $\Xi$ & $:::::$ & $: \because$ & 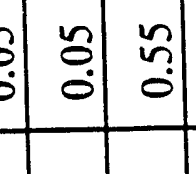 & 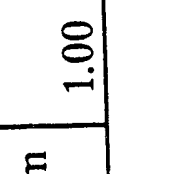 \\
\hline 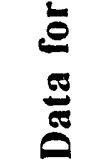 & 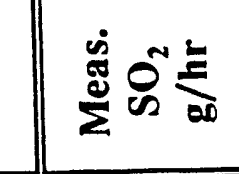 & $\therefore: 2=$ & $=0$ & $g=0$ & \\
\hline 颜 & 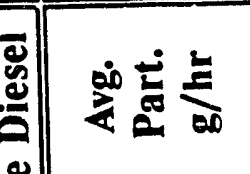 & $D=5=$ & $=n$ & $\Rightarrow=$ & 峷 \\
\hline & 预 & 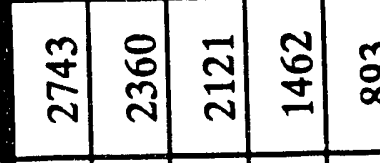 & 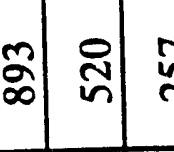 & 䣦: & \\
\hline 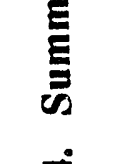 & $8 \frac{\underline{\underline{w}}}{\mathrm{w}}$ & $\overline{0}: 5: 5$ & 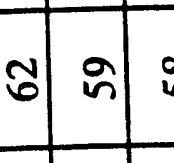 & 0.78 & 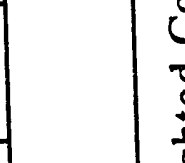 \\
\hline 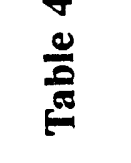 & 呈言 & 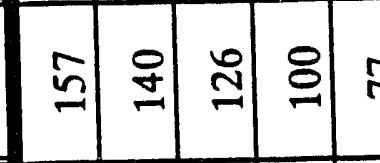 & $=5$ & $6=8$ & \\
\hline & 重 & 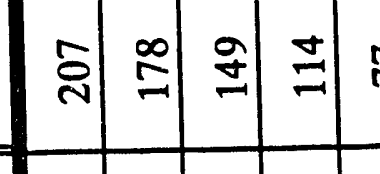 & & $\approx \infty=$ & \\
\hline & $\frac{\bar{s}}{\frac{5}{2}}$ & $\infty-$ & & $-\cong$ & \\
\hline
\end{tabular}




\begin{tabular}{|c|c|c|c|c|c|c|c|c|c|c|c|c|c|}
\hline \multirow{11}{*}{ 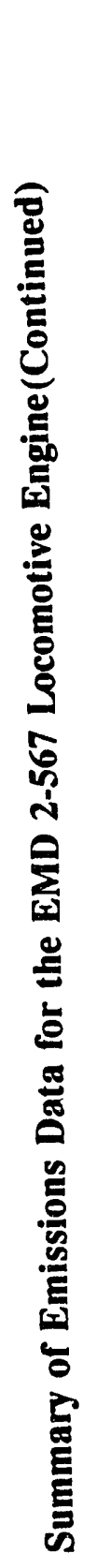 } & \multirow{5}{*}{ 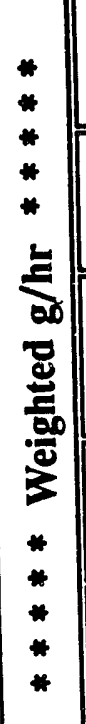 } & $\sum_{\Sigma}^{\mathbb{d}} \infty \delta^{n}$ 놀 & $\approx$ & $\bar{N}$ & 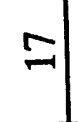 & $m$ & $a$ & $n$ & $m$ & $N$ & \pm & $\stackrel{\infty}{m}$ & $\frac{n}{r}$ \\
\hline & & 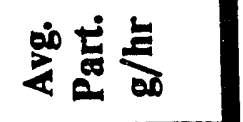 & 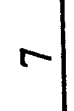 & $m$ & $m$ & $m$ & $N$ & $N$ & $\nabla$ & $\nabla$ & $m$ & $\tilde{b}$ & స్త్ \\
\hline & & $\dot{0} e^{x} e^{x}$ & ฌి & స్ & 오 & 2 & $q$ & $\stackrel{\infty}{\sim}$ & $\approx$ & 의 & 요 & $\stackrel{R}{R}$ & $\stackrel{n}{n}$ \\
\hline & & 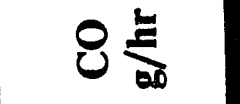 & $\bar{a}$ & ్ి & 의 & $n$ & $m$ & $m$ & $m$ & $m$ & $\approx$ & $\tilde{6}$ & $\ddot{m}$ \\
\hline & & 记 & సి & $\infty$ & 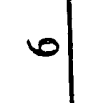 & $n$ & $\nabla$ & $m$ & $m$ & $N$ & $\stackrel{ \pm}{\sim}$ & $\infty$ & $\stackrel{0}{\circ}$ \\
\hline & & 홓 & $\vec{N}$ & $a$ & $r$ & 0 & $\theta$ & $N$ & - & 0 & 0 & 오 & \\
\hline & & $\Sigma$ & $\stackrel{0}{\circ}$ & $\begin{array}{l}n \\
0 \\
0\end{array}$ & $\begin{array}{l}n \\
0 \\
0\end{array}$ & $\begin{array}{l}n \\
0 \\
0\end{array}$ & $\begin{array}{l}n \\
0 \\
0\end{array}$ & $\begin{array}{l}n \\
0 \\
0\end{array}$ & $\begin{array}{l}n \\
0 \\
0\end{array}$ & $\begin{array}{l}n \\
0 \\
0\end{array}$ & 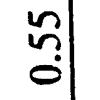 & $\underset{8}{8}$ & \\
\hline & & 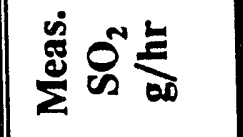 & กี & $\stackrel{\sim}{*}$ & $\vec{m}$ & $\stackrel{n}{\sim}$ & \pm & 5 & $\tilde{\sigma}$ & $\hat{m}$ & $\approx$ & 气ี & \\
\hline & 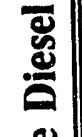 & $\sum^{\infty}$ & $\stackrel{m}{\sim}$ & $\infty$ & $\sqrt[n]{ }$ & กี & $\stackrel{\infty}{\infty}$ & $\bar{\nabla}$ & $\approx$ & 밈 & ப゙ & & 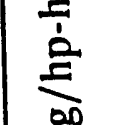 \\
\hline & 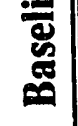 & 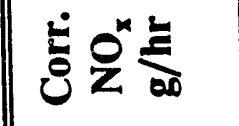 & 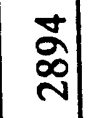 & $\begin{array}{l}\infty \\
\infty \\
\end{array}$ & $\frac{O}{N}$ & 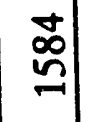 & 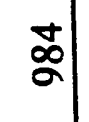 & $\begin{array}{l}\infty \\
0 \\
n\end{array}$ & 요 & న̃ & 의 & & 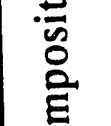 \\
\hline & & 它 & 잉 & 이ํ & $\stackrel{0}{N}$ & 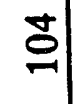 & $\tilde{6}$ & $\bullet$ & $\forall$ & $\dot{n}$ & $q$ & & $\stackrel{\Xi}{ \pm}$ \\
\hline$\frac{0}{0}$ & $\stackrel{2}{m}$ & U로 & జి. & 요 & $\underline{\beth}$ & \& & $m$ & $\stackrel{\infty}{\infty}$ & nn & $\stackrel{m}{q}$ & 8 & & $\overline{\bar{c}}$ \\
\hline & 畐 & 氞 & 우 & 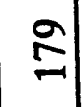 & q & 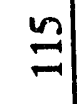 & $\stackrel{\infty}{\sim}$ & $F$ & 9 & $\infty$ & 0 & & \\
\hline & 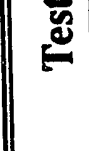 & $\frac{\pi}{\frac{5}{0}}$ & $\infty$ & $\sim$ & 0 & $n$ & $\nabla$ & $m$ & $N$ & -1 & $\frac{0}{.0}$ & & \\
\hline
\end{tabular}




\begin{tabular}{|c|c|c|c|c|c|c|c|c|c|c|c|c|c|}
\hline \multirow{5}{*}{ 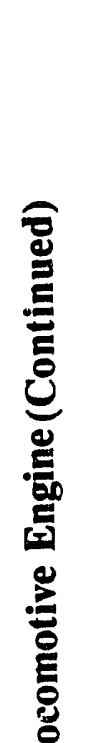 } & \multirow{5}{*}{ 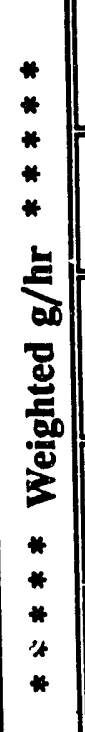 } & 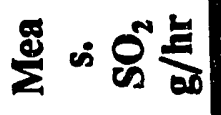 & శె & $\mathbf{N}$ & 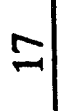 & $\stackrel{N}{-}$ & $\infty$ & 0 & $m$ & $N$ & \pm & $\stackrel{\circ}{n}$ & 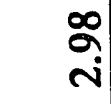 \\
\hline & & 这运炱 & a) & $m$ & $N$ & $N$ & $N$ & $N$ & $m$ & $\forall$ & 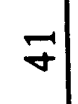 & bิ & m. \\
\hline & & 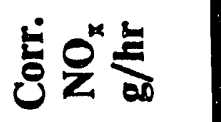 & 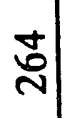 & $\stackrel{\infty}{\sim}$ & $\underset{0}{=}$ & 2 & $\nabla$ & શิ & 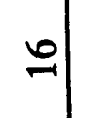 & $a$ & 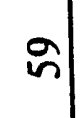 & ని & I \\
\hline & & 일 & શิ & $\approx$ & - & $\nabla$ & $m$ & $m$ & $\nabla$ & $m$ & $\bar{m}$ & ㅇํ & a \\
\hline & & 纪 & $\tilde{\sim}$ & 요 & $n$ & $n$ & $\nabla$ & $m$ & $N$ & $N$ & a) & 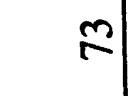 & $\stackrel{\forall}{-}$ \\
\hline గñ & & $\underline{x}$ & $\bar{N}$ & $a$ & $r$ & b & $\nabla$ & $N$ & - & 0 & 0 & 으 & \\
\hline$\sum_{i=1}^{0}$ & & $\Sigma$ & $\frac{0}{0}$ & $\begin{array}{l}n \\
0 \\
0\end{array}$ & $\begin{array}{l}n \\
0 \\
0\end{array}$ & $\stackrel{n}{0}$ & $\stackrel{2}{0}$ & $\begin{array}{l}n \\
0 \\
0\end{array}$ & $\begin{array}{l}n \\
0\end{array}$ & $\stackrel{n}{0}$ & $\tilde{n}$ & $\stackrel{8}{8}$ & \\
\hline 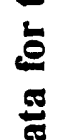 & & 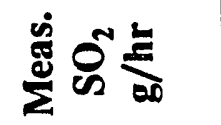 & สิ & $\begin{array}{c}m \\
\infty \\
+\end{array}$ & $\stackrel{m}{m}$ & 足 & 눙 & $\stackrel{\Xi}{\Xi}$ & $\approx$ & $\mathcal{Y}$ & ㄴ. & 㟃 & \\
\hline 哭 & 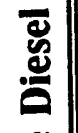 & 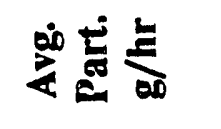 & $\infty$ & nิ & $\stackrel{\infty}{+}$ & P & $\dot{\nabla}$ & \& & $\overline{6}$ & 当 & $\approx$ & & $\frac{\bar{l}}{e^{\circ}}$ \\
\hline $\begin{array}{l}\overline{6} \\
\text { E्ञ }\end{array}$ & 可 & 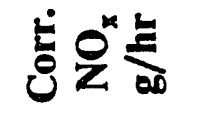 & 苞 & \begin{tabular}{l}
\multirow{2}{n}{} \\
กิ
\end{tabular} & $\frac{m}{m}$ & กิ & $\underset{\infty}{\infty}$ & $\frac{m}{n}$ & $\frac{D}{m}$ & $\infty$ & $\stackrel{\infty}{\circ}$ & & 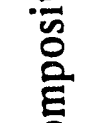 \\
\hline $\bar{E}$ & & 8 롱 & 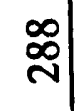 & $\stackrel{\varpi}{\sim}$ & $\underset{\mathcal{N}}{\mathbb{Z}}$ & $\stackrel{O}{T}$ & J゙ & 6 & $\infty$ & $\approx$ & $\curvearrowleft$ & & D্ \\
\hline 党 & 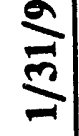 & 经 $\frac{5}{8}$ & ָั & $\check{a}$ & જ் & $\overrightarrow{0}$ & $m$ & సื & 요 & $\vec{\nabla}$ & $\tilde{m}$ & & $\frac{.00}{3}$ \\
\hline & 塸 & 를 & ஜํి & 긴 & q & $\stackrel{m}{=}$ & $\stackrel{0}{*}$ & 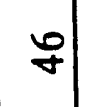 & aి & $\infty$ & 0 & & \\
\hline & $\stackrel{\bar{g}}{\mathscr{E}}$ & $\begin{array}{l}\frac{5}{2} \\
\frac{0}{2}\end{array}$ & $\infty$ & $r$ & 6 & $n$ & $\nabla$ & $m$ & Cv & -1 & $\stackrel{0}{\frac{0}{D}}$ & & \\
\hline
\end{tabular}




\begin{tabular}{|c|c|c|c|c|c|c|c|c|c|c|c|c|c|}
\hline \multirow{4}{*}{ 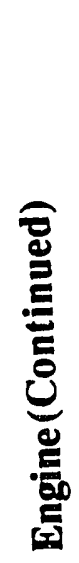 } & \multirow{5}{*}{ 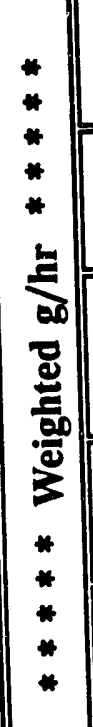 } & 暨的 & त & $\tilde{\sim}$ & $\infty$ & 6 & $\theta$ & -1 & $N$ & -1 & 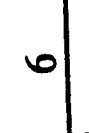 & $\underset{⿱}{*}$ & $\underset{-\infty}{+}$ \\
\hline & & $\sum_{0}^{\infty}$ & $\infty$ & $m$ & $\mathbf{N}$ & $N$ & $N$ & $N$ & $n$ & $\nabla$ & $\mathscr{m}$ & $F$ & $\underset{\nabla}{ت}$ \\
\hline & & $\dot{8} \varrho^{x} \underbrace{k}$ & $\begin{array}{l}\infty \\
\stackrel{\infty}{\sim}\end{array}$ & ్ㅗ & 5 & $\stackrel{\infty}{\sim}$ & $\vec{n}$ & $\stackrel{\infty}{\sim}$ & 0 & $\infty$ & 8 & 6 & $\begin{array}{l}0 \\
\text { in }\end{array}$ \\
\hline & & 일 & 6 & $\because$ & $a$ & $n$ & $m$ & $m$ & $\nabla$ & $m$ & ગ & $\stackrel{\sim}{m}$ & $\begin{array}{l}\text { o } \\
\text { i. }\end{array}$ \\
\hline 플 & & U⿺乚一匕 & \pm & $\infty$ & $n$ & $\forall$ & $m$ & $m$ & $N$ & - & 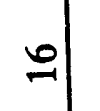 & 미 & $\begin{array}{l}0 \\
\dot{n}\end{array}$ \\
\hline ڤ్ర & & 흘 & $\bar{N}$ & $a$ & $r$ & 6 & $\nabla$ & $N$ & -1 & 0 & 0 & 요 & \\
\hline$\sum_{i=1}^{e}$ & & $\Sigma$ & $\begin{array}{l}0 \\
0 \\
0\end{array}$ & $\check{0}$ & $\begin{array}{l}n \\
0 \\
0\end{array}$ & $\begin{array}{l}n \\
0 \\
0\end{array}$ & $\tilde{0}$ & $\begin{array}{l}\mathscr{0} \\
0 \\
0\end{array}$ & $\begin{array}{l}n \\
0 \\
0\end{array}$ & $\begin{array}{l}\text { ڤ్ } \\
0\end{array}$ & ñ & E⿱ & \\
\hline 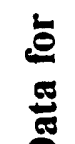 & & 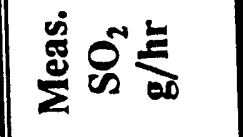 & $\tilde{\sim}$ & $\begin{array}{l}0 \\
7\end{array}$ & 웅 & \pm & 9 & $\stackrel{\circ}{\sim}$ & $\vec{m}$ & $a$ & $\Xi$ & & \\
\hline 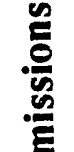 & 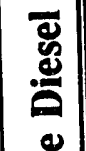 & 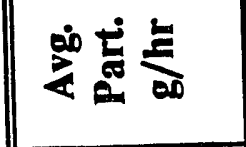 & $\approx$ & in & $\because$ & 7 & $\mathcal{F}$ & q & รू & 밀 & $\stackrel{\infty}{\sim}$ & & $\begin{array}{l}11 \\
\text { 롱 }\end{array}$ \\
\hline E़ & 童 & $\dot{b} e^{x} e^{x}=$ & $\underset{\substack{N \\
\infty}}{\infty}$ & 年 & $\frac{\tilde{J}}{N}$ & 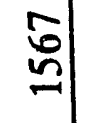 & స్ & $\begin{array}{l}\infty \\
0 \\
\infty\end{array}$ & $\frac{a}{m}$ & กิ & $\stackrel{\infty}{0}$ & & 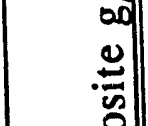 \\
\hline हี & & ○ & $\begin{array}{l}\infty \\
0 \\
0\end{array}$ & $\frac{0}{n}$ & 임 & 의 & J & $\forall$ & $\underset{\nabla}{*}$ & $\curvearrowleft$ & $\stackrel{\sim}{\mathcal{F}}$ & & हี \\
\hline 品 & $\stackrel{7}{2}$ & 놀 & \& & 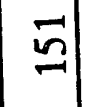 & à & 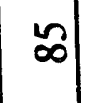 & $\infty$ & $\vec{n}$ & $\vec{\nabla}$ & จิ & ิㅗ & & $\begin{array}{l}\frac{E}{00} \\
\frac{00}{0}\end{array}$ \\
\hline & 苞 & $\hat{x}$ & 옹 & פ & $\underset{⿱}{+}$ & \pm & $\stackrel{\infty}{r}$ & o & 이 & $\infty$ & 0 & & \\
\hline & 总 & $\frac{\mathfrak{c}}{\tilde{e}}$ & $\infty$ & $r$ & 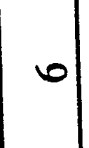 & $n$ & $\nabla$ & $m$ & $\mathbf{N}$ & - & $\stackrel{0}{.}$ & & \\
\hline
\end{tabular}


Table 5. Weighted Composite Brake Specific Emissions Summary for the EMD 2-567 Locomotive Engine

\begin{tabular}{||l||c||c||c||c||c||}
\hline \multicolumn{1}{|c||}{} & PM & HC & CO & NO $_{\mathbf{x}}$ & SO $_{2}$ \\
\hline \multicolumn{4}{|c||}{} & \multicolumn{4}{|c||}{ Composite (g/bhp-hr) } \\
\hline Pre 500-Hour : Baseline 2D & 1.29 & 1.3 & 2.1 & 14.2 & 1.3 \\
\hline Pre 500-Hour : Coal-Lite & 1.26 & 1.6 & 3.3 & 15.3 & 2.8 \\
\hline Post 500-Hour : Coal-Lite & 1.36 & 1.4 & 1.9 & 14.7 & 3.0 \\
\hline Post 500-Hour : Baseline 2D & 1.41 & 1.1 & 2.6 & 15.0 & 1.5 \\
\hline EPA Heavy Duty Diesel Std. : 1994 & 0.1 & 1.3 & 15.5 & 5.0 & NA \\
\hline $\begin{array}{l}\text { Proposed Off-Highway California 8 } \\
\text { Mode : 1995 } \\
: 2000\end{array}$ & $\begin{array}{c}0.4 \\
0.16\end{array}$ & 1.0 & $\begin{array}{c}\text { N.A. } \\
\text { N.A. }\end{array}$ & $\begin{array}{c}6.9 \\
5.8\end{array}$ & $\begin{array}{c}\text { N.A. } \\
\text { N.A. }\end{array}$ \\
\hline
\end{tabular}

significant on the piston and liner of the left cylinder. Wear on the piston of 0.002 ", and on the liner of $0.0057^{\prime \prime}$ is considerably greater than normal. Additionally, the wear area was visually detected, as shown in Figure 11.

Although the wear on the left piston and liner was significant, it does not mean that the wear was induced as a result of running the coal-lite fuel. In fact, other evidence indicates that the wear was not fuel related. This is substantiated by examining the right cylinder piston and liner. Wear here was below the measurable range as expected in 500 hours. Additionally, none of the other components measured indicated significant wear as shown on the dimension sheets (Appendix E).

Except for the left cylinder, visual inspection of all engine components did not indicate any abnormal wear condition. Photographs of the components inspected are presented in Appendix F. Visually, varnish deposits were observed on the injector needle assemblies (see photographs Appendix F). In fact, it was somewhat difficult to remove the needle from the bore due to the varnish deposits. The deposits did not inhibit engine operation but were unusual for 500 hours operation. 


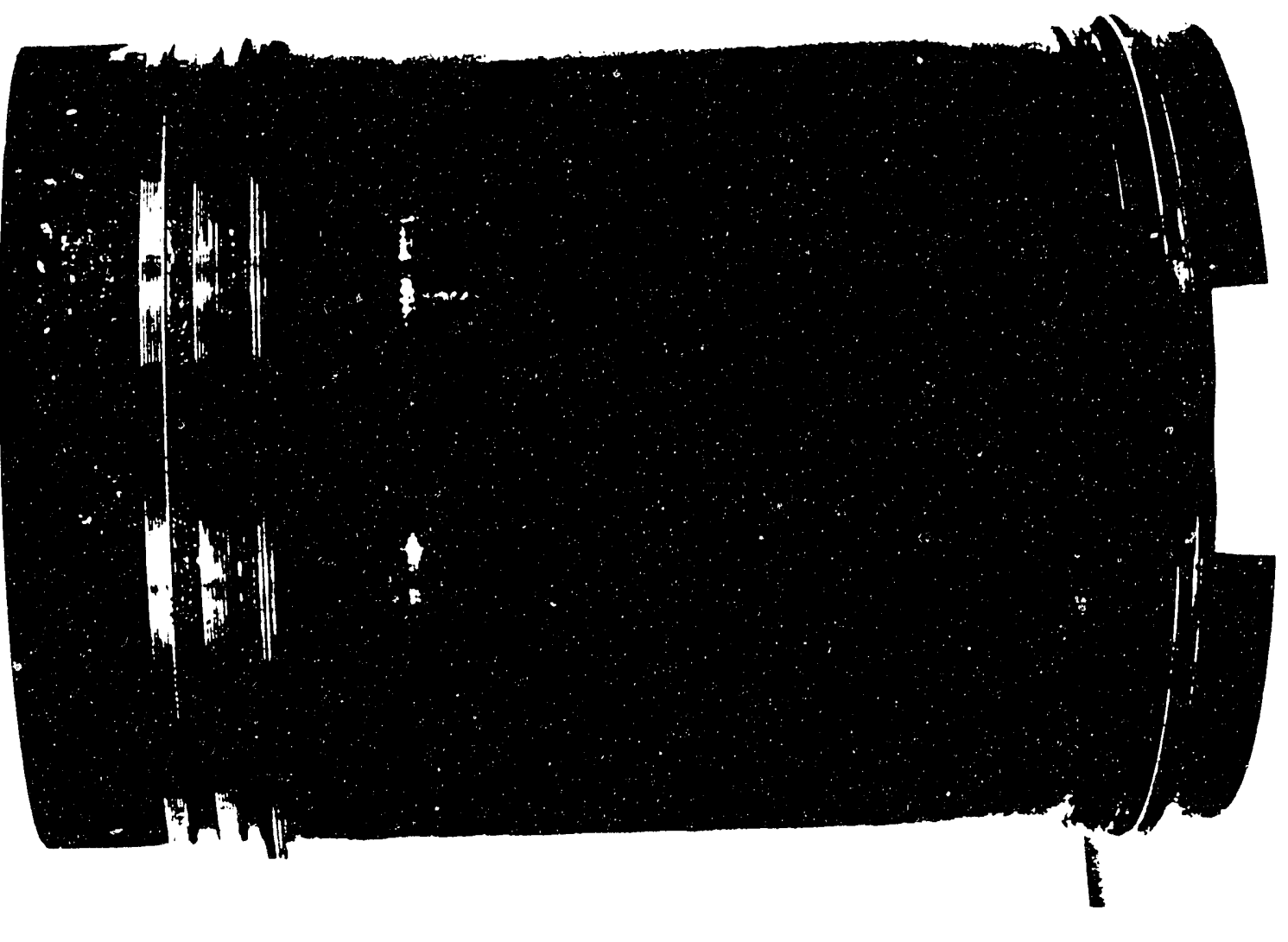

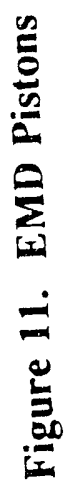

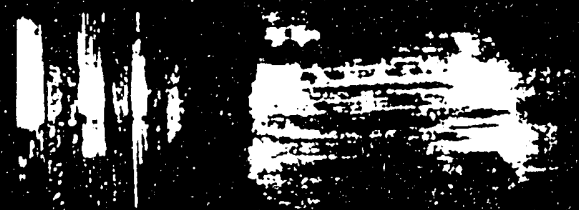

by $:=0$

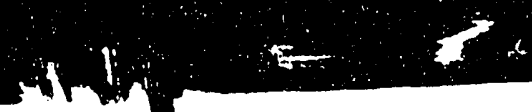




\section{DISCUSSION}

This test was preformed in order to evaluate the performance and emissions of the coal-lite fuel as compared to neat diesel fuel and to determine if any detrimental wear would be observed after 500 hours of operation.

\section{A. Performance Tests}

The performance results indicated that, operationally in the medium-speed diesel engine, the fuel functioned well. No problems were encountered with starting, knock, or general engine operation. However, due to the lower heating value of the fuel and lower thermal efficiency observed, a fuel efficiency penalty of approximately 2 to 4 percent can be expected at the higher notch operation (Notches 5 to 8 ). The thermal efficiency penalty could be a result of a slower burn duration of the coal-lite fuel. Therefore, if this is the case, some of the penalty might be recovered by advancing the injection timing. This would have to be determined experimentally.

\section{B. Wear Tests}

In 500 hours of operation on medium-speed diesel engines, wear measurements can only determine if the fuel tested was catastrophic to the engine. Typically, the wear should be undetectable. Therefore, if wear is measured, then it can be assumed that the fuel is unsatisfactory and should not be consumed for fear of subsequent engine destruction. In this case, significant wear was detected in the left cylinder. The level of this wear, if fuel induced, would condemn the fuel for use in this engine. However, since this level of wear was not detected with any other components it can be assumed that the wear was a function of defective components or the rebuild and not a function of the fuel consumed.

Of some concern is the varnish deposits on the fuel injection components. After 500 hours, the injectors were still functioning properly however the needles were stuck in their bores. Some question exists if this would present a problem with unsatisfactory operation after additional hours of operation.

\section{Emissions Tests}

Based on the limited emissions tests performed on the EMD 2-567B engine, it appears that the coal-lite fuel blend tested did not significantly change the normally regulated exhaust emissions. The only significant difference detected in the measured exhaust emissions was $\mathrm{SO}_{2}$, where the weighted composite brake specific emissions of $\mathrm{SO}_{2}$ were approximately two times higher on the coal-lite fuel as compared to the baseline diesel fuel. However, this result was not unexpected, as the fuel sulfur content of the coal-lite fuel blend was approximately twice that of the baseline diesel fuel. (It should be noted that $\mathrm{SO}_{2}$ emissions are not regulated for 
mobile sources.)

In general, locomotive engine emissions are not regulated is the U.S. The only possible exception to this are the various visible smoke standards in several areas. However, the California Air Resources Board has recently began to study locomotive exhaust emissions, and regulations are anticipated within the next few years for engines built before 1900. At the national level, Congress, through the Clean Air Act Amendments of 1990, instructed the EPA to study locomotive exhaust emissions and to promulgate regulations by 1995 . Those regulations would apply to new engines built since 1990 .

Although the EMD 2-567B engine used in this study is representative of a large number of locomotive engine currently operating in the U.S., the 567 engine design is now two generations old. Significant improvements to the engine design have been incorporated to both significantly improve fuel economy and to reduce exhaust emission levels. Today's state-of-the-art locomotive engines have $\mathrm{NO}_{\mathrm{X}}$ levels of approximately 8-10 g/hp-hr and particulate levels on the order of $0.15-0.25 \mathrm{~g} / \mathrm{hp}$ hr. Given the promising performance, wear and emission characteristics of the coallite in this limited testing on the EMD 2-567B engine, further evaluations may be warranted on full scale locomotive engines. This testing could involve performance and emissions testing on SwRI's 12-cylinder locomotive engines. These engines, an EMD 12-645E3B, and a GE 12-7FDL, are each rated at 2,500 bhp, and are representative of the majority of the existing locomotive fleet in North America. However, these engines are themselves one generation old, and do not incorporate all of the improvements of today's new locomotive engines. In addition to further 12-cylinder locomotive engine tests, a controlled long-term field study of the coal-lite fuel blend may be considered to evaluate the in-service characteristics of the fuel.

\section{CONCLUSIONS}

After 500 hours of operation, performance and wear measurements made on the engine as a result of running on coal-lite fuel did not indicate that the fuel was catastrophic. However, varnish deposits on the fuel injectors should be monitored. If used in rail operation, the coal-lite fuel should be used in controlled field tests for one year until longer term results can be observed. The coal-lite fuel did not significantly change the normally regulated exhaust emissions, but did significantly increase $\mathrm{SO}_{2}$ emissions because of its higher sulfur content. 
APPENDIX A

$$
\text { A-1 }
$$


GASEOOS and/Or PARTICULATE EUISSIONS

IDLE

PROJECT 03-2681-809

TRST DATE $1 / 31 / 91$

DIESEL DF-2

TOLARL NO. 26

SAIPLE TIKE $20 \times 20 \quad .00$ KIN.

BLONER TELP. DEG. F 59.

FOEL FLON,LB/KTH
.09
BLONER COUNTS 47666.

OBS. PONER, BAPP .0
PROGPN $=$ EWD16B

BAROMETER 29.55 IH.BG.

BOKIIDITY 26.71 GRAIYS B20/LB. AIR

LFE 10. 15 BIG. LPE 10.0

SAIPLE TIME 47m 30.00 MIK.

MRAS. EXH. FLON,LB/KIM 16.34

AIR FLON,LB/KIIM $\quad 16.25$

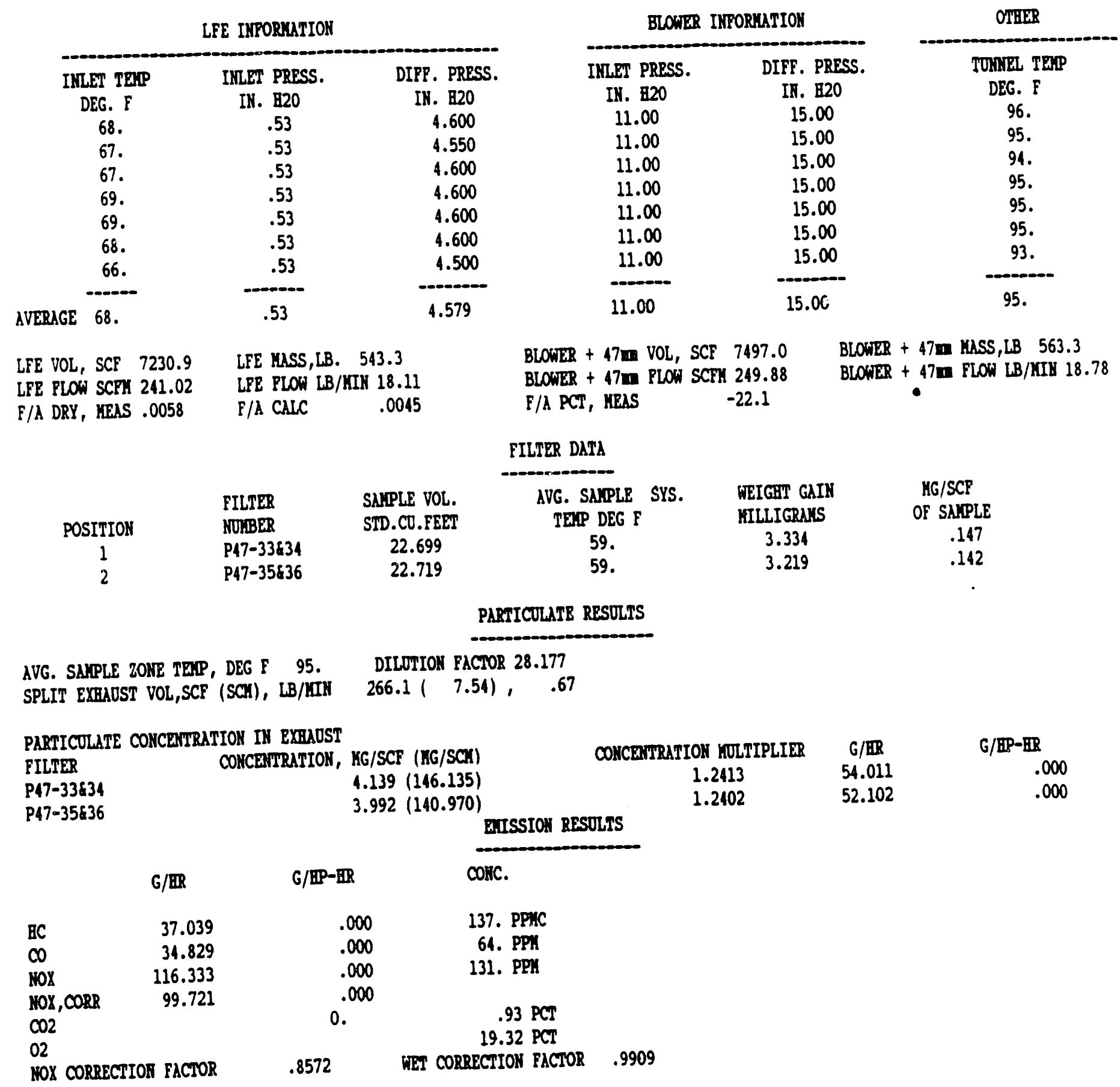


GASEOOS and/Or PAPTICOLATE EIISSIOHS

IOTCE 1

PROJECT 03-2681-809

TRET HO. RON HO. 8

MIGINE HODEL EID 2-567B

TRST CELL LOCO LAB

BCR 1.758

BLONER COUTIS 47682.

OBS. PONER, BEP 8.3
TRST DATE $1 / 31 / 91$

TOSAR 10.26

BLOWRR TERP. DEG. F 61.

PUEL PLON,LB/KIY
DIESRR DP-2

SAIPLE TIRE $20 \times 20 \quad .00 \mathrm{KIH}$.
PROGPAY $=$ DD16B

BAROAETER' 29.55 IN.BG.

BUIIDITY 25.08 GRAIIS B20/LB. AIR

LPE XO. 15 EIG. LPE NO. 0

SAIPLE TIRE 47m $30.00 \mathrm{nIMN}$.

NERS. BXH. FLON,LB/LIN 16.36

AIR FLON,LB/:III

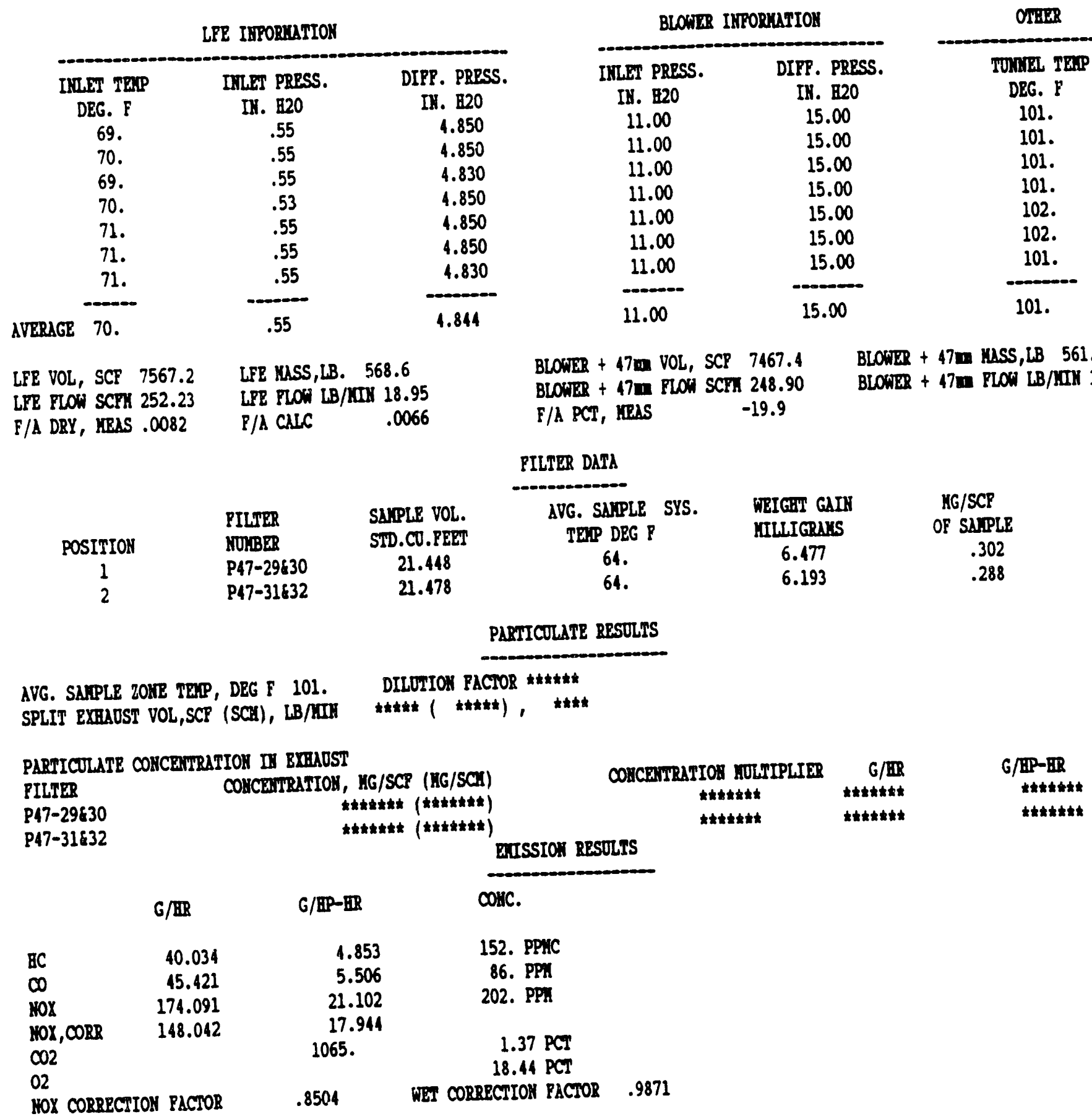


GASEOOS and/Or PAPTICOLATE EISSIONS

HOTCR 2

PROJECT 03-2681-809

TEST DATE $1 / 31 / 91$

DIESEL DF-2

TUNARL HO. 26

SAVPLE TIIT $20 \times 20 \quad .00$ KIN.

BLONER TEIP. DEG. \& 63.

FOEL FLON,LB/KIN
.20
PROCRAY $=$ ED16B

BAPOIETER 29.54 IH.BG.

BOIIDITY 25.56 GRAIMS B20/LB. AIR

LFE 10. 15 EIG. LFE 10. 0

SAMLE TIKE 47m 30.00 MIH.

VIRAS. EXH. FLON,LB/XIN 21.53

AIR FLOW,LB/KIM 21.33

OBS. PONER, BRP 20.0

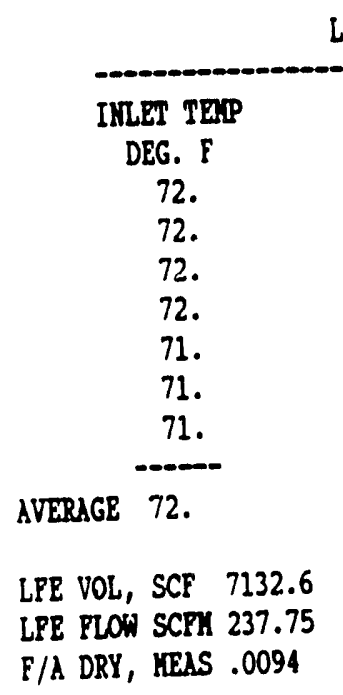

LPE IMTORNATION

IITET PRESS. DIPF. PRESS.

III. 120

.48

III. $\mathrm{E} 20$

.48
.48

4.590

4.590

4.590

4.590

4.570

4.550

4.550

.50

.50

.49

4.576

LFE MASS, LB. 535.9

LPE RLON LB/KII 17.86

F/A CALC
BLONER IMPORUITON

IRLET PRESS.
IN. 120
11.00
11.00
11.00
11.00
11.00
11.00
11.00
-11.00

OTHER

TULAR TEIP

DRG. F

114.

114.

114.

114.

113.

113.

113.

114.
BLOWER + 47w VOL, SCP $7433.9 \quad$ BLONER + 47w WLSS,LB 558.6

BLONER + 47E FLON SCFI 247.80

F/A PCT, TEAS

$-14.6$

FILTER DATA

$\begin{array}{clc} & \text { FILTER } & \text { SAYPLE VOL. } \\ \text { POSITIOH } & \text { NUTBER } & \text { STD.CO.FEET } \\ 1 & \text { P47-25 } \$ 26 & 21.422 \\ 2 & \text { P47-27£28 } & 21.412\end{array}$

PARTICULATE RRSOLTS

AVG. SAIPLE SYS. TEIP DEG F

65.

65.
15.00
BLONER + 47W PLON LB/KIN 18.62

47m. PLON LB/KIIN 18.62

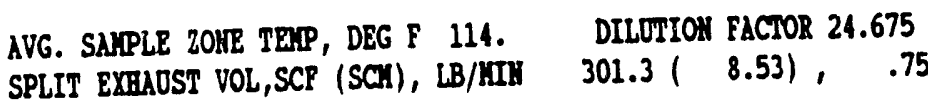

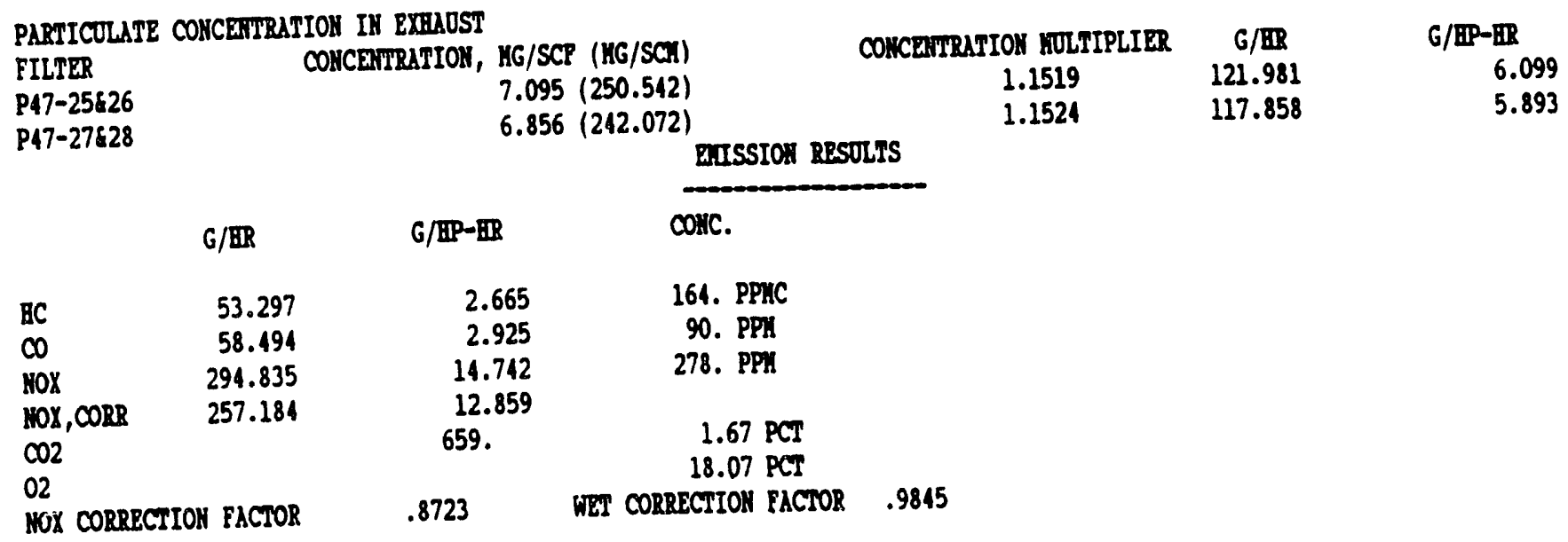




\section{GASEOOS and/Or PARTICOLATE BIISSIONS \\ HOTCE 3 \\ PROJECT 03-2681-809}

TRST DATE $1 / 31 / 91$

DIESEL DP-2

TOMNEL HO. 26

SAIPLE TIKE $20 \times 20 \quad .00$ KIN.

BLONER TERP. DEG. F 66.

FOEL TLON,LB/MTN

.35

BLONTR COUIISS 47656. OBS. POWER, BIP 47.0

$$
\text { PROGRAI = DID16B }
$$

BAROIETER 29.54 IH. BG.

BUIIDITY 29.37 GRAIKS B20/LB. AIR

LPE 10. 15 BIG. LPE 110. 0

SAIPLE TIIE 47m 30.00 IIN.

YTAS. EXH. FLOK,LB/ $/ \mathrm{KIM} 27.80$

AIR FLON, LB/KIN

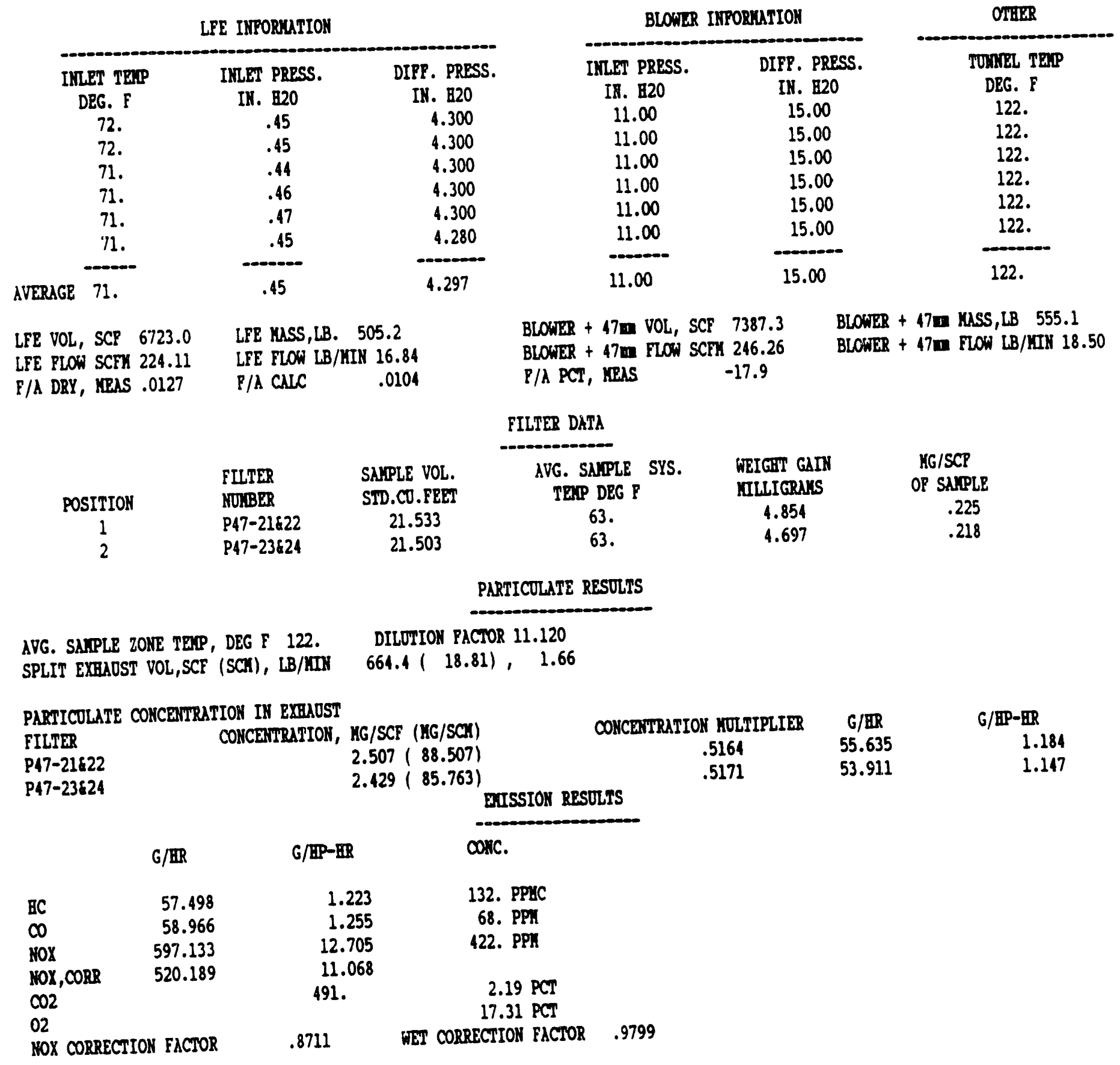


GASEOOS and/Or PARTICOLATE BIISSIOHS

BOTCH 4

PROJECT 03-2681-809

TEST DATE $1 / 31 / 91$

DIESEL DF -2

TUTREL HO. 26

SAIPLE TISE $20 \times 20 \quad .00 \mathrm{KIH}$.

BLONIRR TEIP. DEG. F 67.

FOEL FLON,LB/KIN $\quad .54$
PROGRAY = EID16B

BARONLTER 29.55 IH.BG.

BUIIDITY 25.54 GRAIHS B20/LB. AIR

LPE 10. 15 EXG. LFE 10. 0

SATPLE TIME 47m 21.38 MIH.

KRAS. EXP. FLON,LB/KIH 34.24

AIR FLON,LB/KIH

33.70

BLONIRR COUNITS 33964.
OBS. PONER, BIP 77.0

LFE IRFORYATION

\begin{tabular}{|c|c|c|}
\hline IIRLET TEXP & IMLET PRESS. & DIFF. PRESS. \\
\hline DEG. F & IH. $\mathrm{B2O}$ & IH. 820 \\
\hline 73. & .52 & 4.080 \\
\hline 72. & .52 & 4.080 \\
\hline 72. & .49 & 4.130 \\
\hline 73. & .51 & 4.130 \\
\hline 73. & .52 & 4.150 \\
\hline 73. & .48 & 4.200 \\
\hline GE 73. & .51 & 4.128 \\
\hline
\end{tabular}

BLOWER IHFORUATION

$\begin{array}{cc}\text { IYLET PRESS. } & \text { DIFF. PRESS. } \\ \text { II. B20 } & \text { IH. B20 } \\ 11.00 & 15.00 \\ 11.00 & 15.00 \\ 11.00 & 15.00 \\ 11.00 & 15.00 \\ 11.00 & 15.00 \\ 11.00 & 15.00 \\ 11.00 & -2000\end{array}$

OTHER

TOLAEL TESP

DEG. $F$

123.

123.

123.

123.

124.

123.

123.
LFE VOL, SCF 4591.9 LFE MASS,LB. 345.0

LFE FLON SCFY 214.81 LFE ILON LB/MIH 16.14

F/A DRY, KEAS .0161 F/A CALC .0136
BLONER + 47 E VOL, SCP 5256.2 BLOWER + 47m MLSS,LB 394.9

BLOWER + 47W FLON SCFH 245.88 BLONER + 47W FLON LB/IIH 18.48 F/A PCT, IRAS -15.6

FILTER DATA

$\begin{array}{ccc}\text { AVG. SAIPLE SYS. } & \text { WEIGHT GAIN } & \text { MG/SCF } \\ \text { TEIP DEG P } & \text { MILIIGRNS } & \text { OP SAYPLE } \\ 61 . & 3.639 & .240 \\ 61 . & 3.505 & .231\end{array}$

PARTICULATR RESULTS

AVG. SAYPLE ZOIT TEAP, DEG F 123. DILUTTOH PACTOR 7.913

SPLIT EXEAOST VOL,SCF (SCA), LB/KIIH
$664.2(18.81), 2.33$

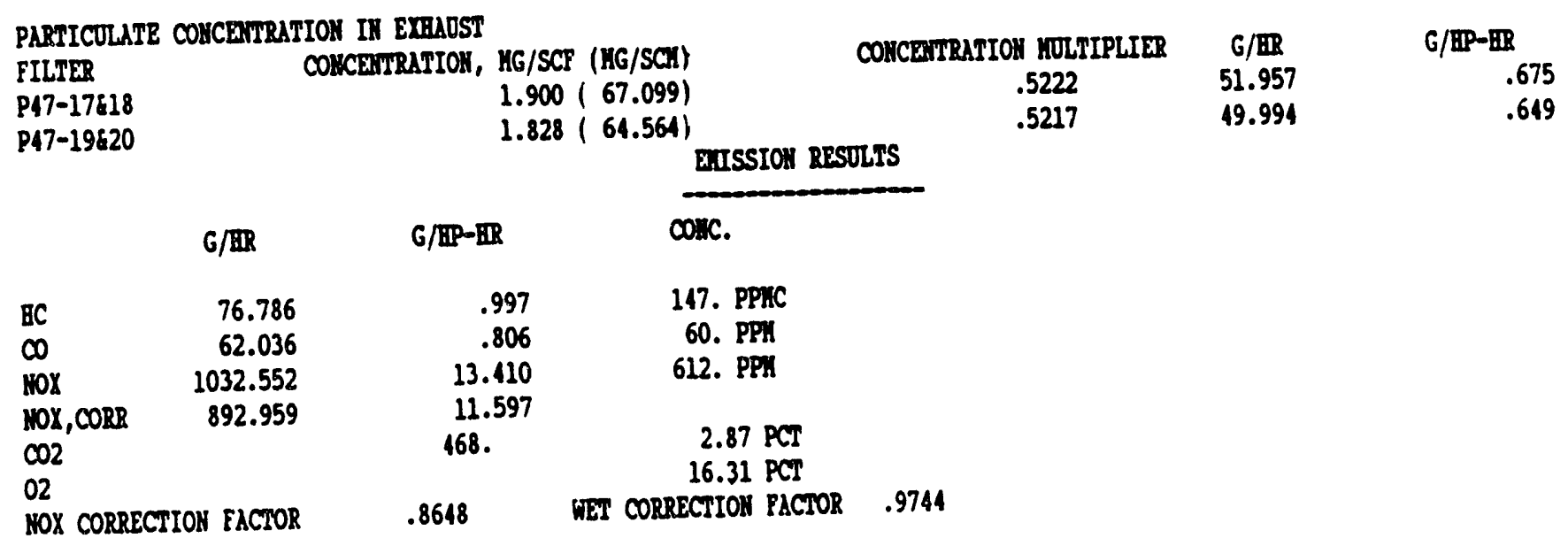


GASEOOS and/or PARTICULATE EIISSIOHS

NOTCH 5

PROJECT 03-2681-809

TEST HO. ROI 110. 4

BIGINE HODEL EID 2-567

TEST CELL LOCO LAB

BCR 1.758

BLONER COUTTS 32889.

OBS. PONER, BHP 114.0
TEST DATE 1/31/91

DIESEL DF-2

IUTREL NO. 26

SAYPLE TIKE $20 \times 20 \quad .00$ MIK.

BLONTRR TEAP. DEG. F 67.

FOEL FLON,LB/KIN $\quad .76$
PROGRAI = EDD16B

BAROIIETER 29.60 IY.BG.

BOIIDITY 23.35 GRAIHS E20/LB. AIR

LFE 10. 15 EIG. LFE 10. 0

SAIPLE TIYR 47m 20.70 MITH.

ITRAS. EXH. FLOW,LB/KIN 38.26

AIR FLON, LB/KIN $\quad 37.50$

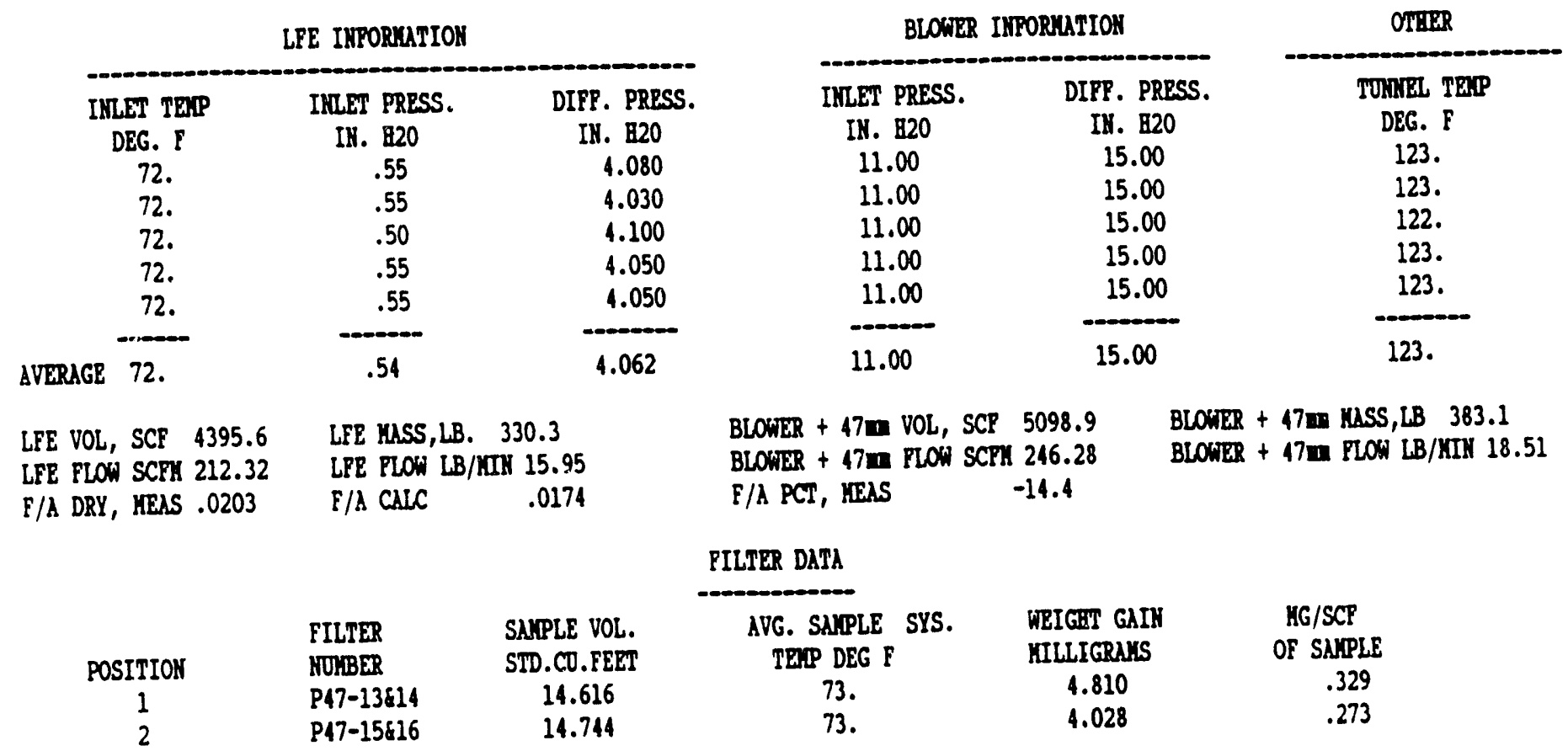

PARTICULATE RESULTS

AVG. SAYPLE 2OKE TEAP, DEG F 123. DILOPIOH PACTOR 7.251

SPLIT BXHAOST VOL,SCP (SCI), LB/KIIY $703.2(19.92), \quad 2.55$

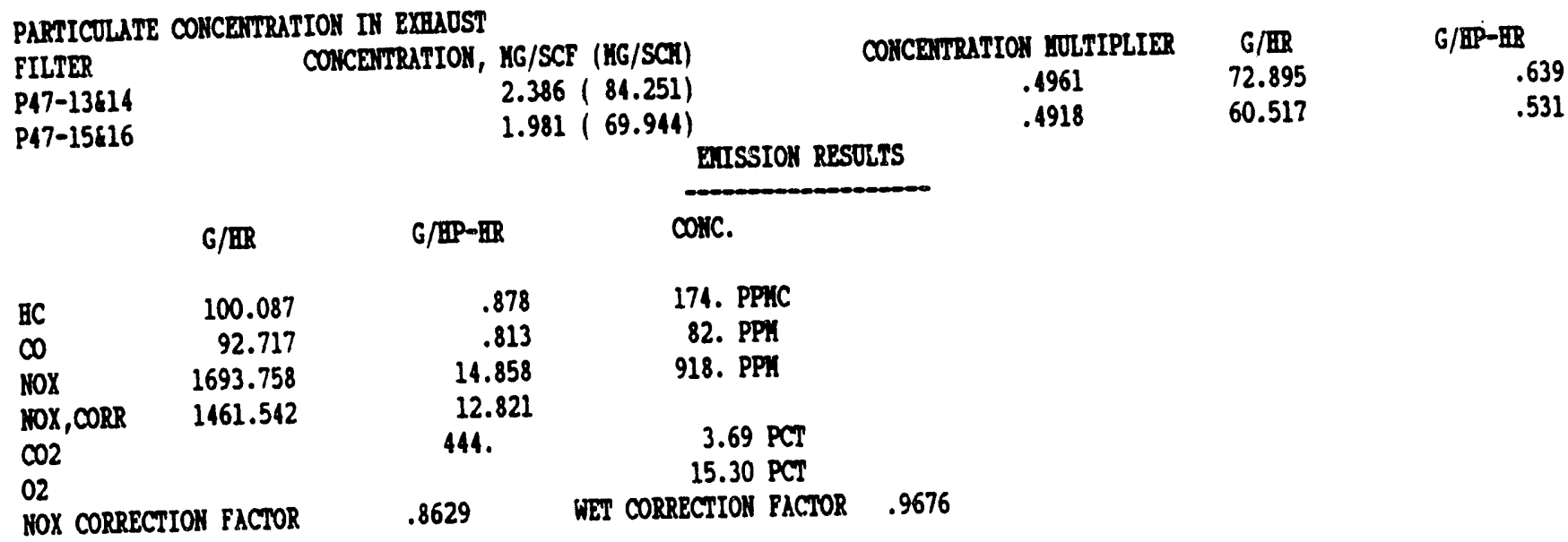


GASEOOS and/Or PARTICOLATE MIISSIONS

NOTCH 6

PROJECT 03-2681-809

PROGRAH = EDD16B

TEST 10.

ROA 1 HO. 3

ENGINE HODEL END 2-567

TEST CELL LOCO LAB

BCR 1.758

BLONER COUTIS 47669.

OBS. PONER, BMP 149.0
TEST DATE $1 / 31 / 91$

DIESEL DP-2

TORLEL HO. 26

SAIPLE TIME $20 \times 20 \quad .00$ MIK.

BLONER TER. DEG. F 67.

FOEL FLON,LB/YIN $\quad .99$
BAPOXETER 29.62 IN.BG.

BOIIDITY 23.31 GRAIKS E20/LB. AIR

LFE 10. 15 MIG. LFE 10. 0

SAYPLE TIME 47m 30.01 KTH.

MEAS. EXH. FLON,LB/KTM 43.69

AIR FLON, LB/MIM $\quad 42.70$

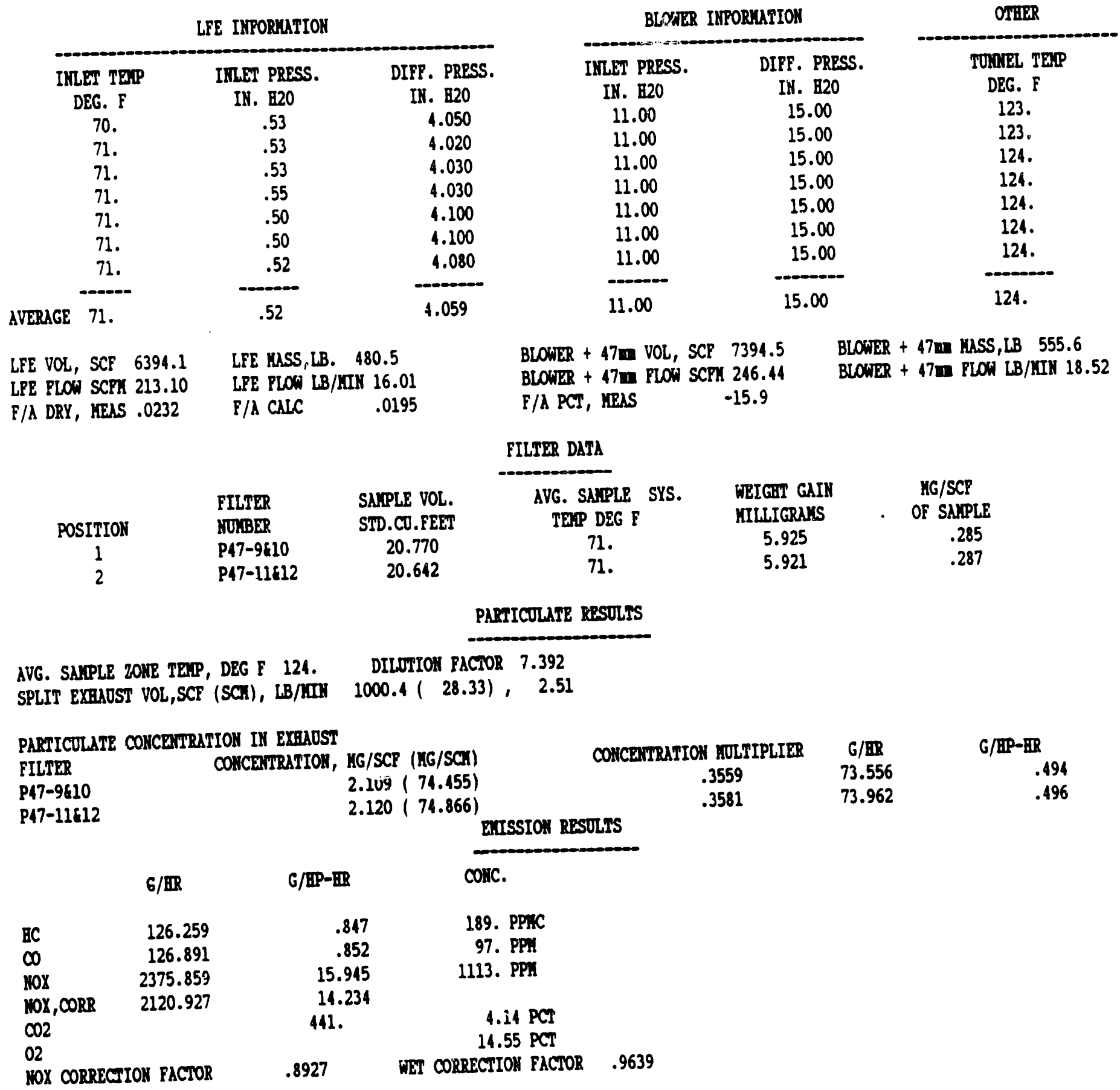


GASEOOS and/Or PAPTICOUATE MISSIONS

HOTCH 7

PROJECT 03-2681-809

TRST DATE $1 / 31 / 91$

DIESEL DP -2

TOIXEL NO. 26

SAMPLE TIYE $20 \times 20 \quad .00$ MIK.

BLONER TERP. DEG. P 66.

FORL FLON,LB/LIN 1.21
PROGRAI $=$ END16B

BAROFIEIER 29.63 IN. BG.

HUNIDITY 24.93 GRAIHS B20/LB. AIR

LPE HO. 15 EIG. LFE HO. 0

SAIPLE TIKE 47m 30.00 YIH.

YRAS. EXE. FLON,LB/KIN 49.79

AIR FLON, LB/IIIN

OBS. POWER, BHP 178.0

LFE IMPORUATIOI

\begin{tabular}{ccc} 
& & \\
\hline IMLET TERP & IRLET PRRSS. & DIPP. PRESS. \\
DEG. F & TH. B20 & IN. E20 \\
72. & .55 & 4.100 \\
72. & .55 & 4.100 \\
71. & .55 & 4.100 \\
71. & .55 & 4.050 \\
71. & .54 & 4.000 \\
71. & .55 & 4.100 \\
71. & .56 & 4.050 \\
\hdashline 71. & .5 & -1.071
\end{tabular}

BLOWER IHPORULTIOH

INLET PRESS
IN. 1820
11.00
11.00
11.00
11.00
11.00
11.00
11.00
0.00
11.00

DIPF. PRESS.

II. 820

15.00

15.00

15.00

15.00

15.00

15.00

15.00

15.00

BLONER + 47m MASS,LB 556.6

BLOWER + 47.1 VOL, SCP 7408.1 BLOWER + 47m FLOW SCPI 246.95 F/A PCT, IEAS

$-7.8$

LPE FLON LB/IIIH 16.0

F/A DRY, IRAS .0250 F/A CALC .0230
OIHER

TOWNEL TRIP

DEG. F

121.

122.

122.

121.

122.

122.

122.

122.
PILTER DATA

$\begin{array}{ccc}\text { AVG. SAYPLE SYS. } & \text { WEIGHT GAIN } & \text { MG/SCT } \\ \text { TEIP DEG F } & \text { MILLIGRALS } & \text { OF SAIPLE } \\ 68 . & 6.432 & .310 \\ 68 . & 6.474 & .312\end{array}$

$\begin{array}{clc} & \text { EILTER } & \text { SAIPLE VOL. } \\ \text { POSITION } & \text { NOIBER } & \text { STD.CO.FEET } \\ 1 & \text { P47-5\$6 } & 20.767 \\ 2 & \text { P47-7\$8 } & 20.757\end{array}$

PARTICULATE RESULTS

AVG. SAMPLE ZONE TEAP, DEG P $122 . \quad$ DILOTION PACTOR 7.383

SPEIT EXHAOST VOL,SCF (SCI), LB/III 1003.4 ( 28.42), 2.51

PARTICULATE CONCENTRATION IN EXHAOST

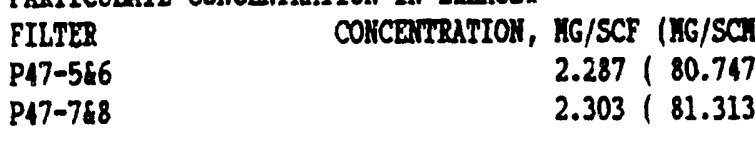

\begin{tabular}{|c|c|c|}
\hline COHCMIRATIOH YULTIPLIER & G/BR & G/HP-ER \\
\hline .3555 & 90.915 & .511 \\
\hline .3557 & 91.552 & .514 \\
\hline
\end{tabular}

B.ISSIOA RRSOLTS

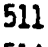

G/ER G/BP-IR CONC.

$\begin{array}{lrr}\text { BC } & 140.413 & .789 \\ \infty & 254.211 & 1.428 \\ \text { 10X } & 2753.695 & 15.470 \\ \text { 10X, CORR } & 2360.537 & 13.261 \\ \text { CO2 } & & 452 .\end{array}$

02

HOX CORRECTION FACTOR
.8572
202. PPHC

188. PPH

1248. PPI

$4.90 \mathrm{PCT}$

$13.79 \mathrm{PCT}$

WEI CORRECTIOH FACTOR .9576 
GASEOOS and/Or PARTICOLATE EIISSIOAS

HOTCH 8

PROTECI 03-2681-809

TEST HO. RON NO. 1

BIGIIE YODEL EID 2-567B

TRST CRLL LOCO LAB

BCR 1.758

BLONTER COUITS 47673.

OBS. POCTER,BLP 206.8
TEST DATE 1/31/91

DIESEL DP-2

TOWEL 110. 26

SAYPLE TIIE $20 \times 20 \quad .00 \mathrm{KIN}$.

BLONTR TETP. DEG. F 67.

FOEL FLON, LB/KIN $\quad 1.45$
PROGRAI = EID16B

BAROILIER 29.62 IH.HG.

HUITDITY 29.21 GRAIIS H20/LB. AIR LPE 10. 15 EIG. LFE 10. 0

SAIPLE TISR 47.1 30.00 KIH.

ITAS. EXH. PLON,LB/STH 55.92

AIR PLON, LB/KIH

54.47

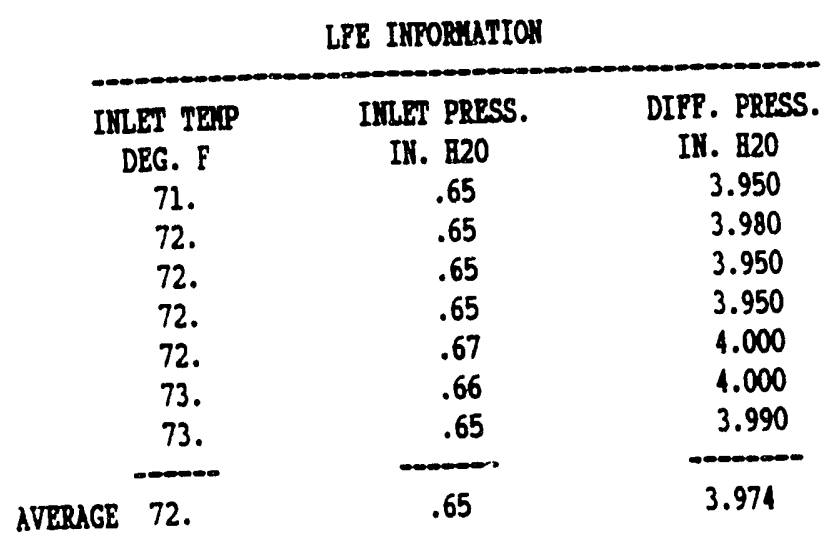

LFE VOL, SCF 6237.2

LPE FLON SCTI 207.91

F/A DRY, IRAS .0267

LIE MLSS,LB. 468.7

LPE FLON LB/ITIN 15.62

F/A CALC

.0266
BLONTR IMEORUATTOA

IRLER PRRSS.
II. 1820
11.00
11.00
11.00
11.00
11.00
11.00
11.00
11.00

BLOWER + 47m VOL, SCF 7394.7

BLONTR + 47m PLON SCPh 246.49

F/A PCT, YEAS

$-.6$

FILTER DATA

AVG. SAIPLE SYS. TEAP DEG I

63.

63.

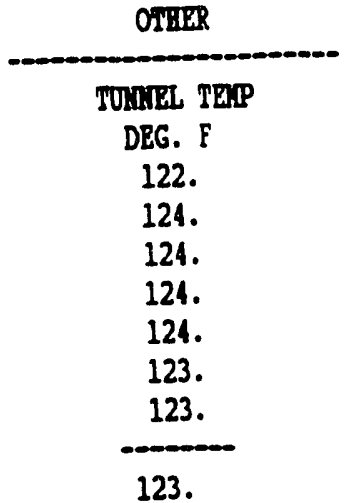

BLONER + 47m MASS, LB 555.6

BLONRR + 47TI. PLON LB/MIH 18.52
DILIEL TEIP

124.

123.

123.
DIFT. PRESS.

II. B20

15.00

15.00

15.00

15.00

15.00

15.00

15.00

15.00
POSITION NOLBER

$1 \quad \mathrm{P} 47-162$

2 P47-3\&4
SATPLE VOL.

STD.CO.FEET

20.444

20.424

WEIGIT GAIY
.MILIGRAYS
6.306
6.473

KG/SCP

OF SAIPLE

.308

.317

PARIICULATE RESULTS

AVG. SAYPLE 2OITE TRIP, DEG P 123. DILOTIOA PACTOR 6.389

SPLIT EXEAOST VOL, SCF (SCH), LB/IIIH $1157.5(32.78), 2.90$

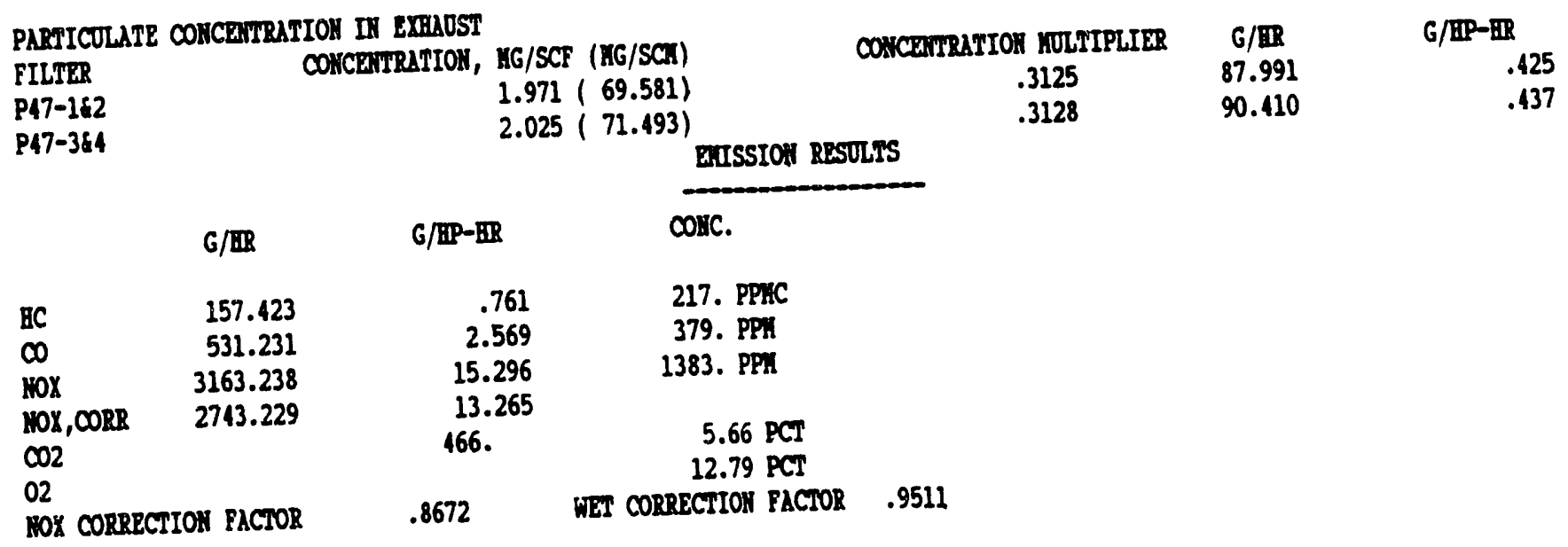


APPENDIX B

B-1 
GASEOOS and/Or PARTICOLATE EISSIONS

IDLE

PROJECT 03-2681-809

TEST 10. ROY HO. 9

ENGINE MODEL END 2-567B

TEST CELL LOCO LAB

BCR 1.656

BLONTRR COUITS 47620.

OBS. PONER,BIP .0
TEST DATE 2/4/91

DIESEL COAL LIQO

TONISL NO. 26

SAYPLE TIME $20 \times 20 \quad .00 \mathrm{KIH}$.

BLOAER TERT. DEG. F 73.

FOEL FLON, LB/KIN $\quad .09$
PROGRAY = ED16B

BAPOLETER 29.26 IH.BG.

BOUIDITY 61.20 GRAIHS B20/LB. AIR

LFE HO. 15 BIG. LFE HO. 0

SAYPLE TIYE 47m 30.00 KIN.

IRAS. EXR. FLON,LB/KIM 16.54

AIR FLON, LB/KIM $\quad 16.45$

\begin{tabular}{|c|c|c|}
\hline \multicolumn{3}{|c|}{ LPE IMPORHATION } \\
\hline IRLET TERP & IMLET PRRSS. & DIPP. PRESS \\
\hline DEG. $F$ & II. $\mathrm{B} 20$ & II. 120 \\
\hline 81. & .60 & 4.600 \\
\hline 81. & .60 & 4.620 \\
\hline 80. & .60 & 4.600 \\
\hline 80. & .60 & 4.600 \\
\hline 80. & .60 & 4.600 \\
\hline 80. & .60 & 4.600 \\
\hline 80. & .60 & 4.600 \\
\hline GE 80. & .60 & 4.603 \\
\hline
\end{tabular}

BLOWT
IKLET PRESS
IN. B20
10.00
10.00
10.00
10.00
10.00
10.00
10.00
10.00

BLONER + 47m VOL, SCP 7248.5

BLONER + 47m FLON SCPI 241.62

F/A PCI, MEAS

$-24.7$

PILTER DATA

$\begin{array}{clc} & \text { FILTER } & \text { SAIPLE VOL. } \\ \text { POSITIOH } & \text { NOIBER } & \text { STD.CO. FEET } \\ 1 & \text { P90-69870 } & 22.559 \\ 2 & \text { P90-71872 } & 22.549\end{array}$

LFE MASS,LB. 518.6
LFE PLON LB/YTH 17.29

F/A CALC $\quad .0043$
PLON SCFI 230

P90-71872

22.549
ONRR IHPORMTIOH

\begin{tabular}{c} 
DIPP. PRESS. \\
IN. 120 \\
14.00 \\
14.00 \\
14.00 \\
14.00 \\
14.00 \\
14.00 \\
14.00 \\
\hline 14.00
\end{tabular}

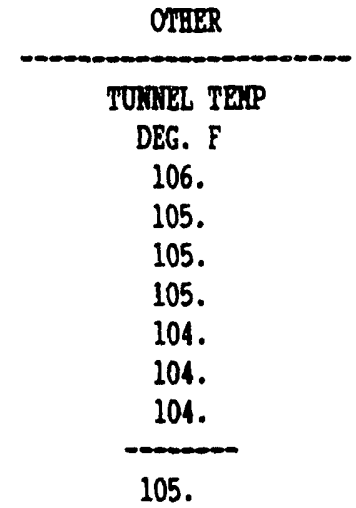

BLOWER + 47m MLSS, LB 544.6 BLONER + 47W RLON LB/KIN 18.15

\section{IIG/SCF \\ OF SAJPLE \\ .236}

.229

PARTICOLATE RESULTS

AVG. SAYPLP 20KR TERT, DEG P 105.

DILOTIOH PACTOR 20.937

SPLIT EXHAOST VOL,SCP (SCI), LB/III 346.2 ( 9.80$), \quad .87$

PARTICOLATE COKCEITRATIOH IN EXHAOST

FILTER

P90-69670

P90-71872
COHCEMTRATIOR, MG/SCP (MG/SCH)
$1.941(174.477)$
$4.800(169.471)$

CONCEATTRTIOH NULTIPLIER
.9281
.9285

G/BP

65.274

63.401
G/EP-ER

.000

\section{EIISSIOA RESULTS}

G/ER G/HP-HR CONC.

$\begin{array}{lr}\text { BC } & 43.968 \\ \text { CO } & 18.824 \\ \text { WOX } & 112.095 \\ \text { YOX, CORR } & 106.862 \\ \text { CO2 } & \\ \text { O2 } & \\ \text { HOX CORRECTIOH FACTOR }\end{array}$

HOX CORRECTIOH FACTOR

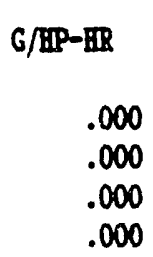

0.

colic.

157. PPIC

86. PPY

121. PPII

$.89 \mathrm{PCT}$

$19.20 \mathrm{PCT}$

.9533 WET CORRECTIOH FACTOR .9904 
HOTCH 1

PROJECT 03-2681-809

TRST KO. ROI HO. 8

EIGIME YODEL END 2-567B

TEST CELL LOCO LAB

BCR 1.656

BLONER COOITS 45450 .

OBS. PONER, BHP 8.3

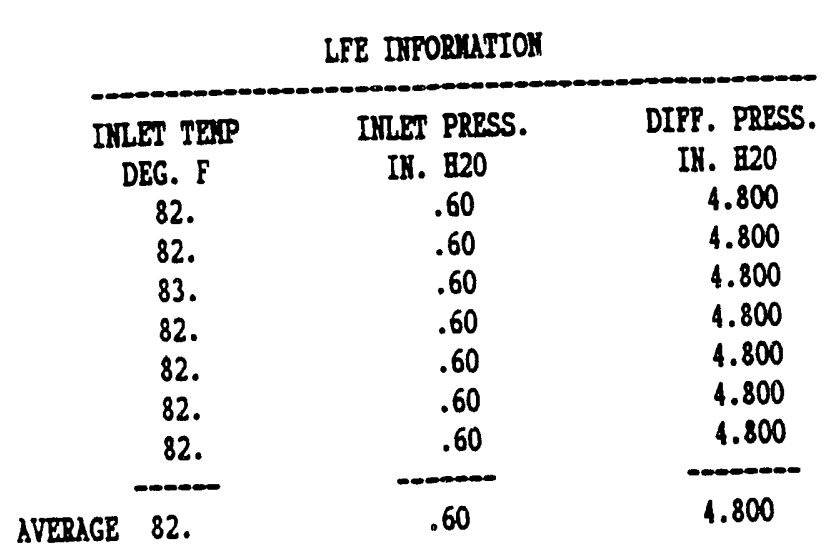

LPE VOL, SCP 6811.1

LPE FLON SCPI 237.90

F/A DRY, MRAS .0086

LFE MLSS,LB. 511.8

LFE PLON LB/LII 17.88

F/A CALC
TRST DATE 2/4/91

DIESEL COAL LIQD

TUixil N0. 26

SAYPLE TIIE $20 \times 20 \quad .00$ KIN.

BLONER TEIP. DEG. F 75.

FUEL PLON,LB/KIN .13
PROCRAY = EDD16B

BAROLETER 29.25 III. BG.

BOIIDITY 116.95 GRAIHS E20/LB. AIR

LPE 10.15 BIG. LPE 10.0

SAIPLE TIIE 47m $28.63 \mathrm{KIH}$.

MRAS. EXP. FLON,LB/KIHY 16.00

AIR FLON,LB/LII

15.87

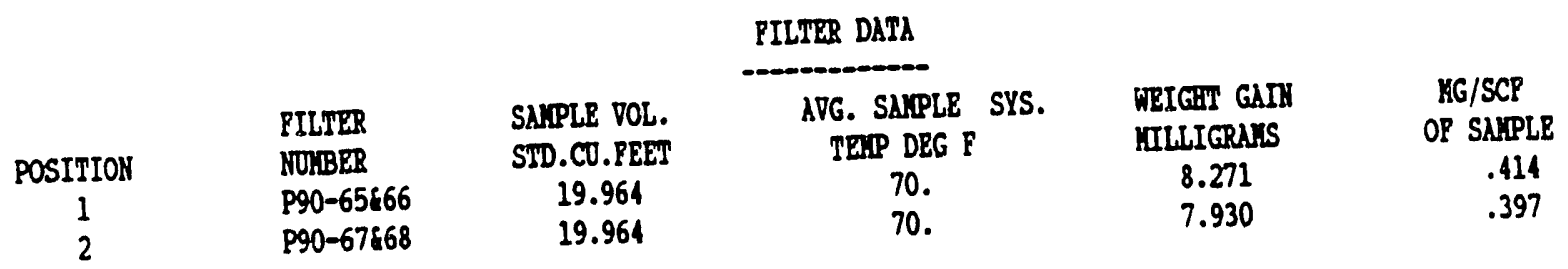

AVG. SAYPLE 2ONR TEAP, DEG P 111.

PARICOLATR RESULTS

BLONER IMFORUATIOS

\begin{tabular}{cc}
\hline IILET PRRSS. & DIPP. PRRSS. \\
II. B20 & II. B20 \\
10.00 & 14.00 \\
10.00 & 14.00 \\
10.00 & 14.00 \\
10.00 & 14.00 \\
10.00 & 14.00 \\
10.00 & 14.00 \\
10.00 & 14.00 \\
\hline 10.00 & -0.00 \\
\hline
\end{tabular}

OIRER

TOAREL TEIP

DEG. F

112.

112.

111.

111.

111.

111.

111.

111.
BLONER + 47m VOL, SCP 6885.9 BLONER + 47m MLSS,LB 517.4

BLONER + 47m PLON SCPH 240.51 BLONER + 47w FLON LB/KIIN 18.07
F/A PCT, VIEAS -21.2

ILTTRR DATA

SPLIT EXEMOST VOL,SCP (SCI), LB/LII

DILOTIOA PACTOR ktktkt

tatk ( tktk)， tkt

\section{PAPTICULATE CORCEYTRATION II EXHAOST \\ FILTER \\ P90-65\&66 \\ P90-67868 \\ COHCETIRATIOH, MG/SCP (HG/SCT) \\ thtwatk (twttatk)

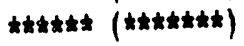

\begin{tabular}{lr} 
& \multicolumn{1}{l}{ G/BR } \\
BC & 43.466 \\
CO & 54.393 \\
NOX & 164.835 \\
NOX, CORR & 191.510 \\
CO2 & \\
O2 & \\
HOX CORRECTION PACTOR
\end{tabular}

\section{BIISSION RESOLIS}

conic.

169. PPHC

105. PPI

195. PPI

1.1618
19.951

23.180

1074.

G/EIP-BIR

5.261
6.583
19.951
23.180
74.

\section{$1.41 \mathrm{PCT}$ \\ 18.57 PCT}

GET CORPRCTION FACTOR .9841

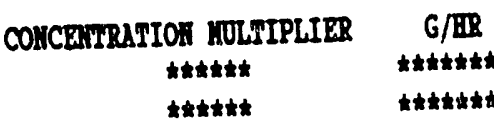

G/HP-IR

tktktkt

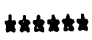


GASEOOS and/or PARTICOLATE EMISSIOKS

HOTCR 2

PROJECT 03-2681-809

PROCRAI = BRD16B

TEST HO. ROR 110.7

EMGIME HODEL EID 2-567

TRST CELL LOCO LAB

BCR 1.656

BLONER COOHIS 44781.

OBS. POWER, BEP 19.3
TRST DATE 2/ 4/91

DIESEL COAL LIQD

TOLARL 10. 26

SAPLE TIIE $20 \times 20 \quad .00 \mathrm{KIH}$.

BLOWER TEIP. DEG. E 78.

FORL FLON,LB/LIN
BAPOHETER 29.24 IN. BG.

HOIIDITY 73.16 GRAINS B20/LB. AIR

LPE H. 15 ENG. LPE MO. 0

SAIPLE TIRE 47m $28.20 \mathrm{KIH}$.

MEAS. EXH. PLOW,LB/IIIN 21.37

AIR FLOH,LB/HIY 21.17

LFE IMPORMTIOH

\begin{tabular}{|c|c|c|}
\hline $\begin{array}{c}\text { INLET TERP } \\
\text { DEG. } \mathrm{F}\end{array}$ & $\begin{array}{l}\text { IHLET PRESS. } \\
\text { IN. B20 }\end{array}$ & $\begin{array}{c}\text { DIPF. PRESS. } \\
\text { IH. } \mathrm{B} 20\end{array}$ \\
\hline 84. & .55 & 4.480 \\
\hline 84. & .55 & 4.500 \\
\hline 84. & .55 & 4.470 \\
\hline 83. & .55 & 1.430 \\
\hline 83. & .55 & 4.430 \\
\hline 84. & .55 & 4.460 \\
\hline 83. & .55 & 4.480 \\
\hline GE 84. & .55 & 4.464 \\
\hline
\end{tabular}

LFE VOL, SCF 6232.3

LPE FLOW SCFI 220.98

LFE MASS,LB. $\quad 468.3$

LFE PLON LB/KIN 16.60

F/A DRY, YEAS .0097

F/A CALC

.0080

BLOWN
INLET PRRSS
IK. B20
10.00
10.00
10.00
10.00
10.00
10.00
10.00
-10.00

BLONTR IHFORIATION

\section{DIFP. PRESS.}

II. 1220

14.00

14.00

14.00

14.00

14.00

14.00

14.00

14.00

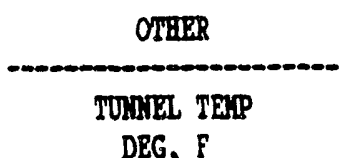

DEG. $F$

123.

123.

123.

123.

123.

123.

123.

123.

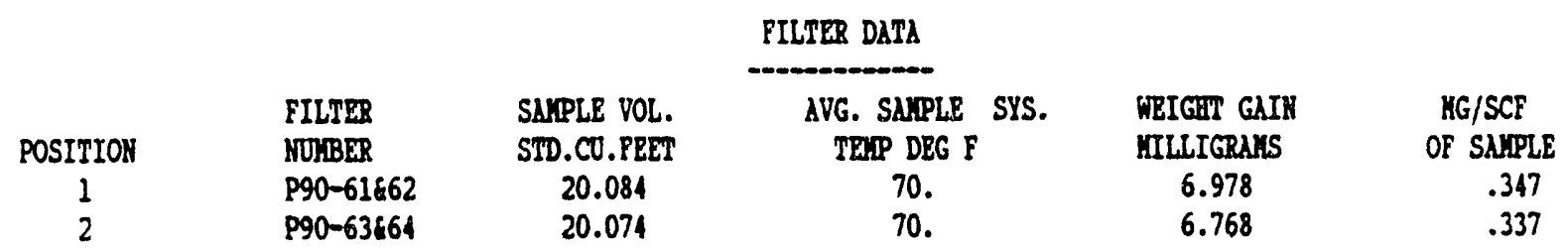

PARTICULATE RESULTS

AVG. SAYPLE 2OKE TEIP, DEG P 123. DILOTIOH FACTOR 13.181

SPLIT EXHAOST VOL,SCF (SCI), LB/YIH 511.7 ( 14.49), 1.36

PARTICULATE COYCEMTRATIOH II EXRAOST

$\begin{array}{lr}\text { FILTER } & \text { CORCETTRATIOH, HG/SCP (HG/SCA) } \\ \text { P90-61662 } & 4.580(161.704)\end{array}$

P90-63864

$4.580(161.704)$
$4.444(156.914)$

BLOWER + 47m VOL, SCP 6744.0

BLONER + 47m KLSS,LB 506.7

BLONER + 47 FLON SCPM 239.12 BLONER + 47m FLON LB/KII 17.97

BLONEB + 47w TLON SCTA 239.12

\section{EIISSIOA RBSULTS}

$\begin{array}{cc}\text { COICERTRAYIOH YOLTIPLIER } & \text { G/ER } \\ .6563 & 78.154 \\ .6566 & 75.839\end{array}$

G/EP-ER

4.045

3.925

CONC.

$\begin{array}{lr}\text { BC } & 52.801 \\ \infty & 64.435 \\ \text { NOX } & 312.182 \\ \text { HOX, CORR } & 308.971 \\ \text { CO2 } & \\ 02 & \end{array}$

HOX CORRECTIOH FACTOR

G/EP- IR

160. PPHIC

97. PPII

2.733
3.335

288. PPI

15.991

697.

$1.67 \mathrm{PCI}$

18.07 PCT

.9897 WET CORRECTIOK PACTOR .9835 
GASEOOS and/or PARTICOLATE MISSIOHS

NOTCR 3

PROJECT 03-2681-809

TRST DATE $2 / 4 / 91$

DIESEL COAL LIQO

TOIARL 10. 26

SAYPLE TIYE $20 \times 20 \quad .00$ KIH.

BLONER TEIP. DEG. I 78 .

FOEL FLON, LB/KIK

.35

OBS. POMER, BRP 46.6

LFE IMPORUATION

\begin{tabular}{|c|c|c|}
\hline IIRET TEIP & IMLET PRESS. & DIFP. PRESS. \\
\hline DEG. F & IN. 820 & IH. $\mathrm{B} 20$ \\
\hline 85. & .65 & 4.330 \\
\hline 84. & .65 & 4.330 \\
\hline 84. & .65 & 4.330 \\
\hline 84. & .65 & 4.310 \\
\hline 85. & .65 & 4.320 \\
\hline 85. & .65 & 4.330 \\
\hline 85. & .65 & 4.330 \\
\hline GE 85 & .65 & 4.326 \\
\hline
\end{tabular}

LFE VOL, SCF 6410.6

LPE FLW SCFI 213.69

LFE MASS,LB. 481.7

LFE PLON LB/KTH 16.06

P/A DRY, MEAS .0130

F/A CALC

.0113

PROGRAY $=$ ED16B

BAROIETERR 29.24 IN.EG.

BOIIDITY 82.18 GRAIKS B20/LB. AIR

LPE 10. 15 ENG. LPE NO. 0

SA.PLE TIIR 47E 30.00 KIH. VIRAS. EXH. FLON,LB/KIN 27.83

AIR FLON, LB/YII $\quad 27.48$

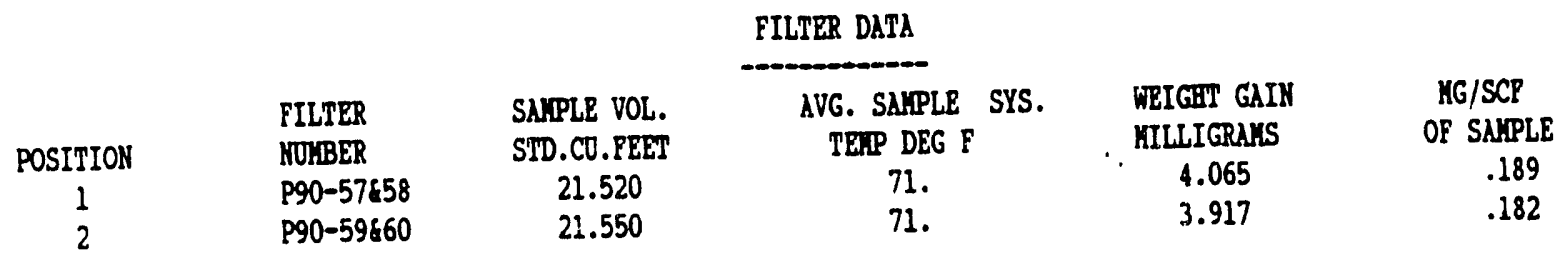

PAPTICULATE RESULTS

AVG. SAIPLE ZORE TEMP, DEG F 122. DILUTIOH FACTOR 9.866

SPLIT EXHAOST YOL,SCF (SCI), LB/HIN $723.1(20.48), 1.81$

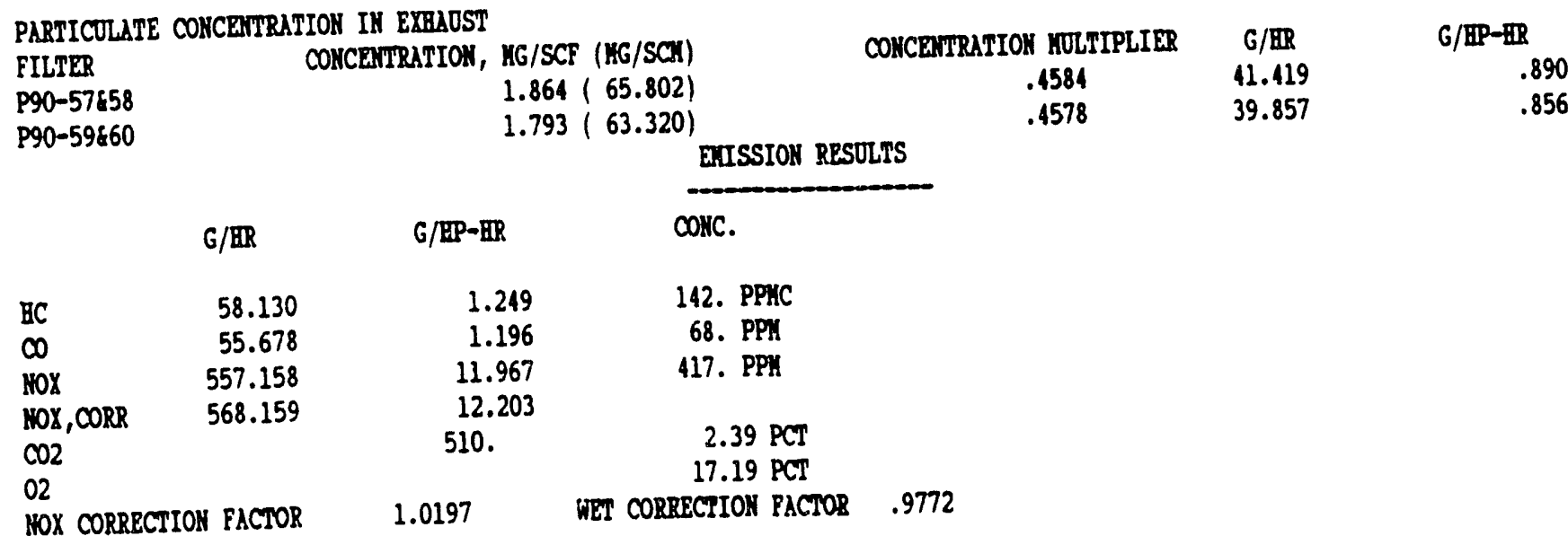


GASEOOS and/Or PARTICOLATE EIISSIOIS

NOTCH 4

PROJECT 03-2681-809

TEST HO. ROH HO. 5

ENGINE MODEL END 2-567B

TEST CELL LOCO LAB

BCR 1.656

BLONER COONIS 25849.

OBS. POWER, BAP 77.9
TEST DATE 2/ 4/91

DIESEL COAL LIQO

TOWARL NO. 26

SAIPLE TIME $20 \times 20 \quad .00 \mathrm{KIN}$.

BLONER TERP. DEG. F 78.

FOEL FLON,LB/HIN
.55
PROGRAH = ED16B

BAROYETER 29.24 IK.EG.

BUIIDITY 78.45 GRAIKS B20/LB. AIR

LPE NO. 15 EHG. LFE NO. 0

SAIPLE TIME 47m 16.29 IIN.

NEAS. EXH. FLON,LB/KIN 34.42

AIR FLOH,LB/KIM $\quad 33.87$

\begin{tabular}{ccc}
\multicolumn{3}{c}{ LFE INPORUATION } \\
\hline IKLET TERP & IKLET PRESS. & DIFP. PRESS. \\
DEG. F & IN. B20 & IN. H20 \\
85. & .67 & 4.090 \\
85. & .67 & 4.090 \\
85. & .67 & 4.100 \\
85. & .67 & 4.100 \\
84. & .67 & 4.100 \\
AVERAGE 85. & .07 & -.096
\end{tabular}

\begin{tabular}{cc}
\multicolumn{2}{c}{ BLONER IRTORMATIOR } \\
\hline IRLET PRESS. & DIPR. PRESS. \\
IN. B20 & IN. H20 \\
11.00 & 15.00 \\
11.00 & 15.00 \\
11.00 & 15.00 \\
11.00 & 15.00 \\
11.00 & 15.00 \\
\hline 11.00 & -200
\end{tabular}

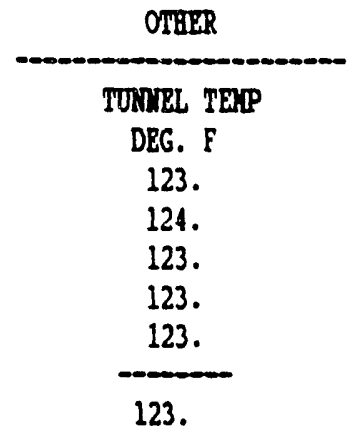

LPE VOL, SCF 3300.8

LFE FLOW SCPI 202.67

F/A DRY, MEAS .0165

LFE MASS,LB. 248.0 LPE PLON LB/LIH 15.23 F/A CNLC

.0142
BLOWER + 47m VOL, SCF 3872.7 BLOWER + 47m MLSS,LB 291.0

BLOWER + 47m PLOH SCPI 237.79 BLOWER + 47n FLON LB/KII 17.87

$P / A$ PCT, YEAS -13.7

FILTER DATA

AVG. SAXPLE SYS. TEIP DEG P

71.

71.

$P 90-55 \& 66$
12.006

SAIPLE VOL.

11.958

WEIGHT GAIN
MTLIGRAYS
2.491
2.451

MG/SCP

OF SAIPLE

.207

.205

PARTICOLATE RRSOLTS

AVG. SAIPLE ZONE TEAP, DEG F 123. DILOTIOH FACTOR 6.772

SPLIT EXHAOST VOL,SCF (SCH), LB/III $571.9(16.20), 2.64$

PARTICOLATE COHCEMTRATIOH IN EXAAOST

FILTER

P90-53\&54

$P 90-55666$

COKCaIIRATIOH, $\mathrm{HG} / \mathrm{SCF}$ (HG/SCH)

$1.405(49.609)$

$1.388(49.010)$

G/IR

$\begin{array}{lr}\text { BC } & 73.457 \\ \text { CO } & 64.526 \\ \text { 10X } & 973.960 \\ \text { 110X, CORR } & 984.411 \\ \text { CO2 } & \\ 02 & \end{array}$

HOX CORRECTIOH FACTOR
G/EP-EIR

$$
\begin{array}{r}
.943 \\
.829 \\
12.509 \\
12.614 \\
476 .
\end{array}
$$

1.0107

\section{BISSION RESULTS}

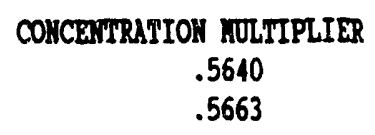

G/ER

38.617

38.151

conic.

145. PPHC

64. PPH

592. PPI

$3.03 \mathrm{PCT}$

$16.18 \mathrm{PCT}$

WET CORRECTIOH FACTOR $\quad .9724$
G/EP-BR

.496

.490 
TEST HO. RON NO. 4

ENGINE YODEL EID 2-567B

TEST CELLL LOCO LAB

- ECR 1.656

BLONER CONTTS 39095.

OBS. PONER, BIPP 114.7
TEST DATE $2 / 4 / 91$

DIESEL COAL LIQO

TURELL NO. 25

SAIPLE TIRE $20 \times 20 \quad .00$ MIN.

BLONER TEIP. DEG. F 78.

FOEL FLON, LB/KII

.77
BAROIIETER 29.26 IN.BG.

BUSIDITY 78.38 GRAIIS H20/LB. AIR

LFE 10. 15 DIG. LPE 110.0

SAIPLE TIIE 47m 24.63 NIN.

IIPAS. EXR. FLON,LB/KIK 39.57

AIR FLON,LB/KMI $\quad 38.80$

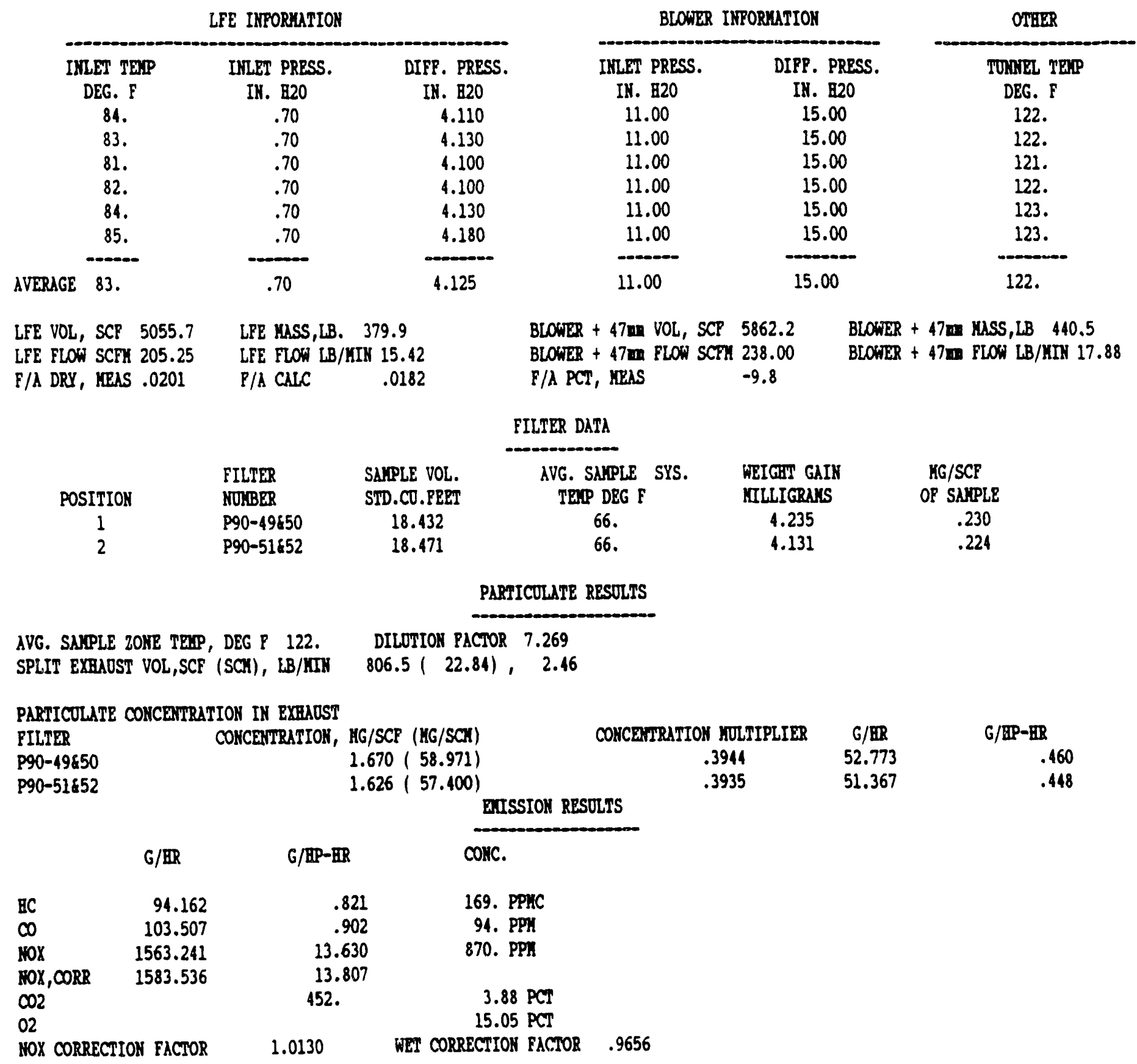




\section{GASEOOS and/Or PARTICULATE EIISSIONS \\ HOTCA 6 \\ PROJECT 03-2681-809}

TRST DATE $2 / 4 / 91$

DIESEL COAL LIQO

TOINEL HO. 26

SAIPLE TIME $20 \times 20 \quad .00$ MIN.

BLONTR TELP. DEG. F 78.

FORL FLON,LB/KII $\quad 1.01$
PROCRAY = DID16B

BAROIETER 29.28 IN.HG.

BUIIDITY 82.46 GRAIHS B20/LB. AIR

LPE MO. 15 EIG. LPE HO. 0

SAIPLE TIIE 47m 30.00 KIN.

MIRS. BXH. FLON,LB/MIM 47.21

AIR PLON, LB/KIK

46.20

BLONER COOIISS 47647 .

OBS. POWER, BHPP 148.6

\begin{tabular}{ccc}
\hline IILET TEAP & INLET PRESS. & DIPP. PRESS. \\
DEG. F & IH. B20 & II. B20 \\
89. & .75 & 4.200 \\
90. & .75 & 4.200 \\
89. & .75 & 4.180 \\
89. & .75 & 4.180 \\
89. & .75 & 4.200 \\
89. & .75 & 4.190 \\
89. & .75 & 4.200 \\
\hdashline 89. & .75 & -1.193
\end{tabular}

BLONER IMFORUATIOH

IRLET PRESS
II. 120
11.00
11.00
11.00
11.00
11.00
11.00
11.00
-000
11.00

DIPP. PRRSS.

II. $\mathrm{B2O}$

15.100

15.00

15.00

15.00

15.00

15.00

15.00

15.00

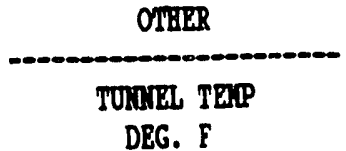

121.

122.

122.

122.

122.

122.

122.

122.
LPE MASS,LB. 461.2 F/A CALC

.0219 LFE FLON LB/KIN 15.37
LPE VOL, SCF 6138.2

LFE FLON SCFI 204.61

E/A DRY, YEAS .0221

$$
\begin{array}{ll}
\text { BLOWER + 47m VOL, SCP } & 7150.3 \\
\text { BLONER + 47m PLOW SCM } 238.34 \\
\text { F/A PCT, YEAS } & -1.0
\end{array}
$$

\section{FILTER DATA}

$\begin{array}{clc} & \text { FILTER } & \text { SAYPLE VOL. } \\ \text { POSITION } & \text { NOIBER } & \text { STD.CO. FEET } \\ 1 & \text { P90-45\$46 } & 22.553 \\ 2 & \text { P90-47\$48 } & 22.611\end{array}$

\section{PARTICULATE RESULTS}

AVG. SAYPLE ZONE TEMP, DEG P 122.

DILOTIOH PACIOR 7.065

SPLIT EXEAOST VOL,SCF (SCA), LB/KIH 1012.1 ( 28.66), 2.54

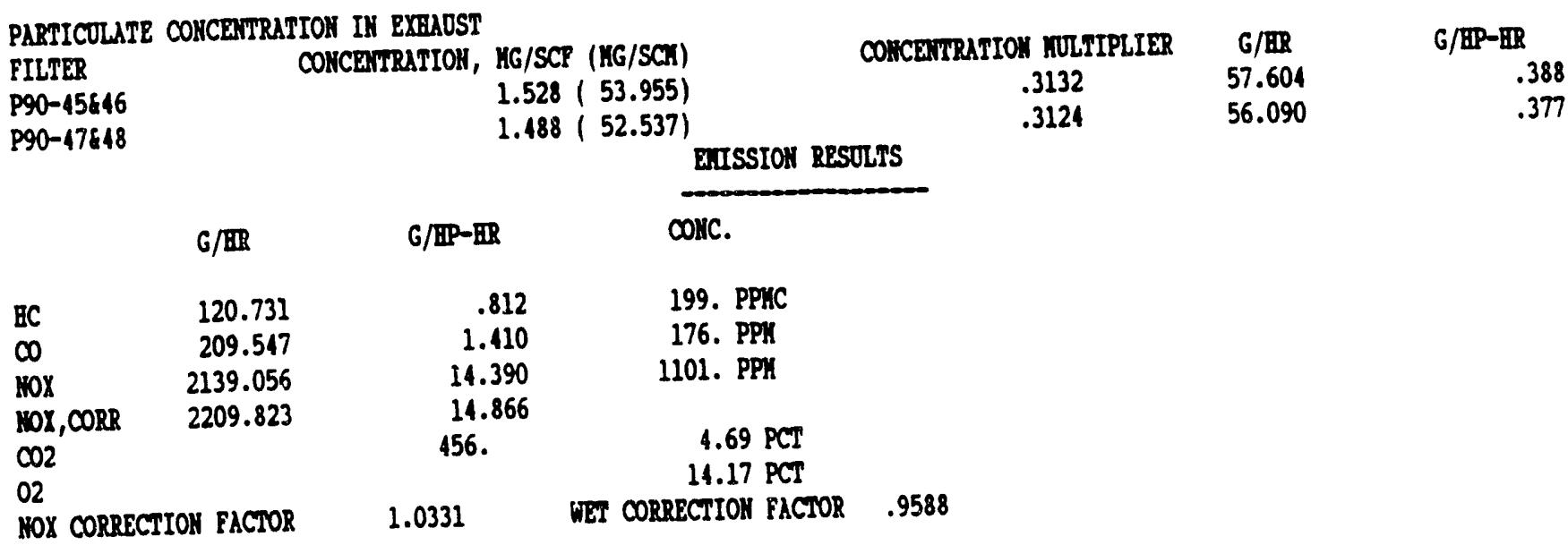

\section{MG/SCF \\ OP SANPLE \\ .216 \\ .211}

4.878

4.762 
GASEOOS and/Or PARTICOLATE EAISSIOHS
HOTCH 7

PROJECT 03-2681-809

TEST N0. ROH 10. 2 TEST DATE $2 / 4 / 91$

EIGIXE HODEL EDD 2-567B

TEST CELL LOCO LAB

1 BCR 1.656

BLONER COUITS 43728.

OBS. PONER, BRP 178.8
DIESEL COAL LIQO

TURTEL HO. 26

SANPLE TIME $20 \times 20 \quad .00$ KIH.

BLONER TELP. DEG. F 78 .

FUEL FLON,LB/KTH $\quad 1.25$
PROGRA $=$ DID16B

BARONETER 29.30 IN. BG.

BUIDITY 76.87 GRAIKS B20/LB. AIR

LPE MO. 15 EIG. LFE MO. 0

SAYPLE TIIR 47m 27.53 MIN.

MEAS. EXH. FLOW, LB/KIY 53.48

AIR PLON, LB/KIH

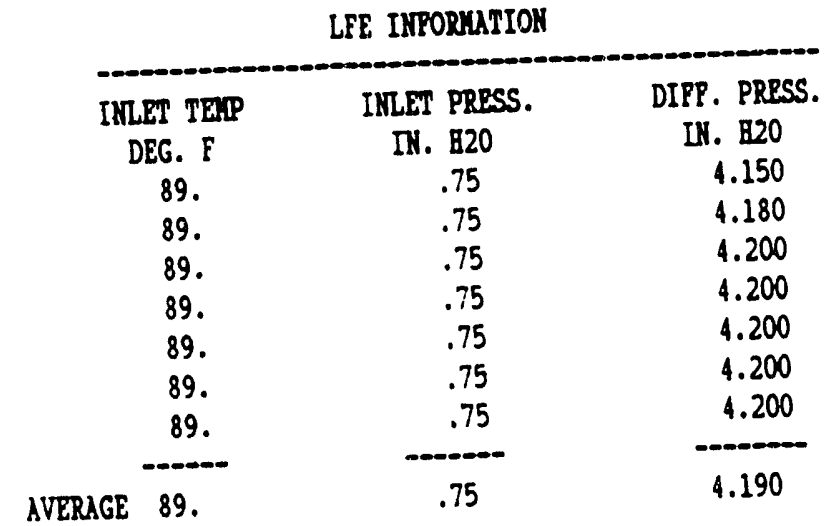

LFE VOL, SCF 5635.6

LPE PLOW SCFY 204.71

F/A DRY, IEAS .0242
LFE MASS,LB. 423.5

LFE FLON LB/KIIN 15.38 E/A CALC

.0250

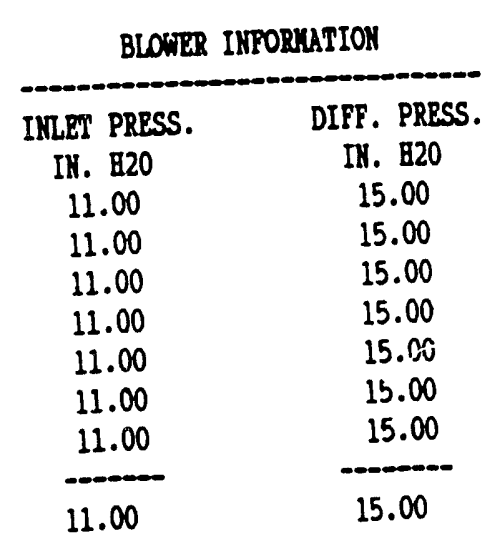

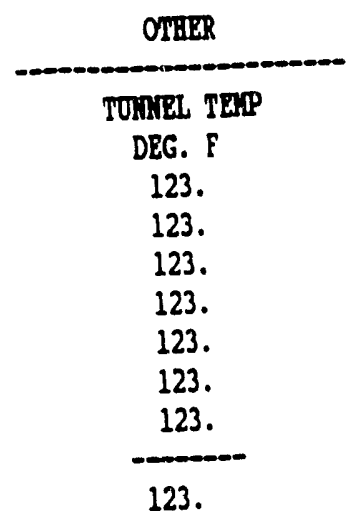

BLONER + 47m VOL, SCF 6566.3

BLONER + 47w PLON SCPI 238.51

F/A PCT, MEAS

BLONER + 47m MUSS,LB 193.4

BLOWER + 47w FLON LB/KIH 17.92

FILTER DATA

\begin{tabular}{|c|c|c|c|c|c|}
\hline & $\begin{array}{l}\text { FILTER } \\
\text { NOIBER }\end{array}$ & $\begin{array}{l}\text { SAMPLE VOL. } \\
\text { STD.CO.FEET }\end{array}$ & $\begin{array}{l}\text { AVG. SAMPLE SYS. } \\
\text { TEMP DEG P }\end{array}$ & $\begin{array}{l}\text { WEIGHT GAIN } \\
\text { MILLIGRANS }\end{array}$ & $\begin{array}{l}\text { KG/SCP } \\
\text { OF SAYPLE }\end{array}$ \\
\hline & $\begin{array}{l}\text { NUIBER } \\
\text { P90- } 11 \& 42\end{array}$ & 20.356 & 77 & 4.662 & .229 \\
\hline $\begin{array}{l}1 \\
2\end{array}$ & $P 90-43844$ & 20.385 & 77. & 4.551 & .223 \\
\hline
\end{tabular}

PARTICULATE RESULTS

AVG. SAYPLE ZORE TEP, DEG F 123. DILOTION PACTOR 7.055

SPLIT EXHAOST VOL,SCF (SCA), LB/KIH $930.7(26.36), \quad 2.54$

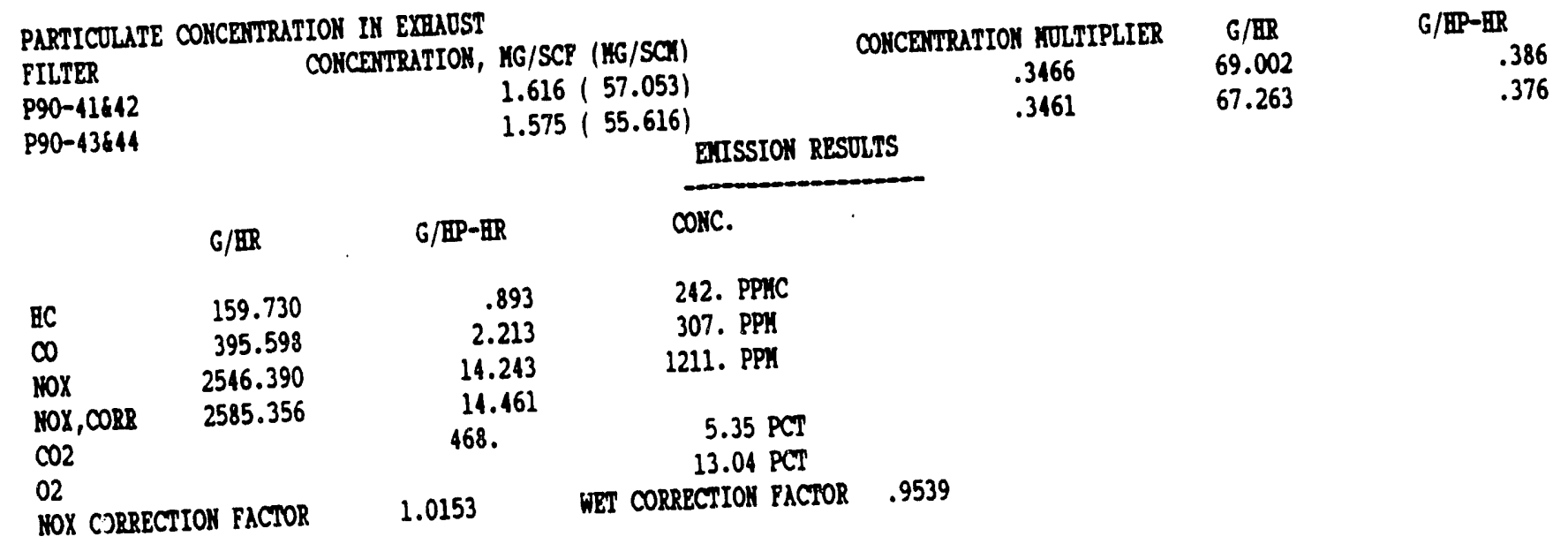


HOTCE 8

PROJECT 03-2681-809

TEST NO. ROH 110. 1

EMGIRE YODEL DID 2-5ETB

TRST CELL LOCO LAB

HCR 1.656

BLOWER COONITS 32104.

OBS. PONTR,BLP 206.3
TEST DATE $2 / 4 / 91$

DIESEL CONL LIQO

TOLITLL HO. 26

SAYPLE TIIE $20 \times 20 \quad .00 \mathrm{KIH}$.

BLONER TETP. DEG. F 78 .

FOEL FLON,LB/KIM $\quad 1.51$
PROGRAY $=$ EDI6B

BAROIETER 29.29 IN.BG.

BOUIDITY 87.52 GRAINS B20/LB. AIR

LFE 10. 15 EIG. LPE 110. 0

SANPLE TIME $47 \mathrm{~m} 20.20 \mathrm{mIn}$.

IREAS. EXH. FLOM,LB/IIM 60.01

AIR FLON,LB/IIN $\quad 58.50$

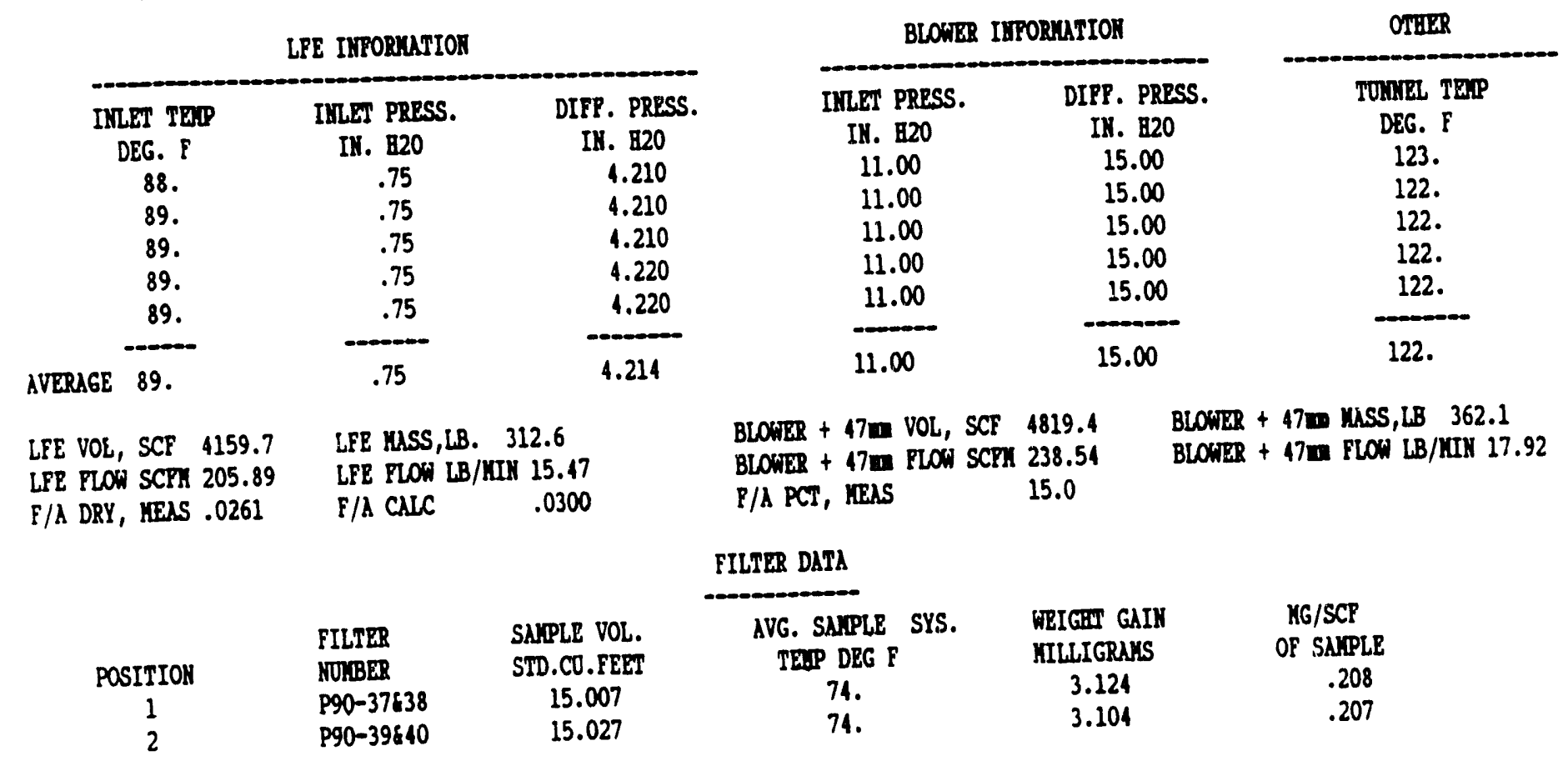

PARTICOLATE RRSOLTS

AVG. SAIPLE ZONE TEAP, DEG P 122. DILOTIOA PACTOR 7.305

SPLIT EXBAOST VOL,SCF (SCH), LB/MII 659.7 ( 18.68), 2.45

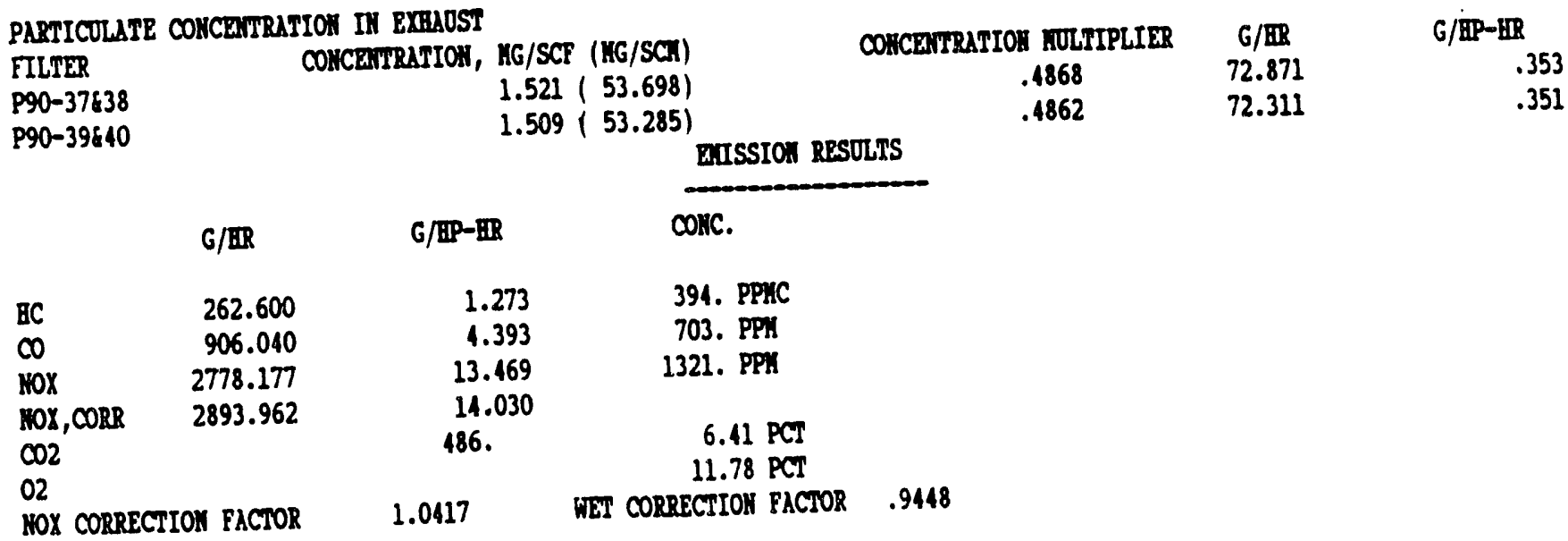


APPENDIX C

C-1 
GASEOOS and/Or PARTICOLATE EMISSIOHS

IDLE

PROJECT 03-2681-809

PROG2AN = DDD16B

TEST HO. ROH HO. 9

EIGIKE YODEL EDD 2-567B

TEST CELL LOCO LAB

BCR 1.656

BLOWER COONTS 26124.

OBS. POWER, BIPP .0
TEST DATE $2 / 26 / 91$

DIESEL COAL LIQO

TOANEL NO. 26

SAYPLE TIYE $20 \times 20 \quad .00$ MIH.

BLONIRR TETP. DEG. F 63. FOEL FLOW,LB/KIN

.9

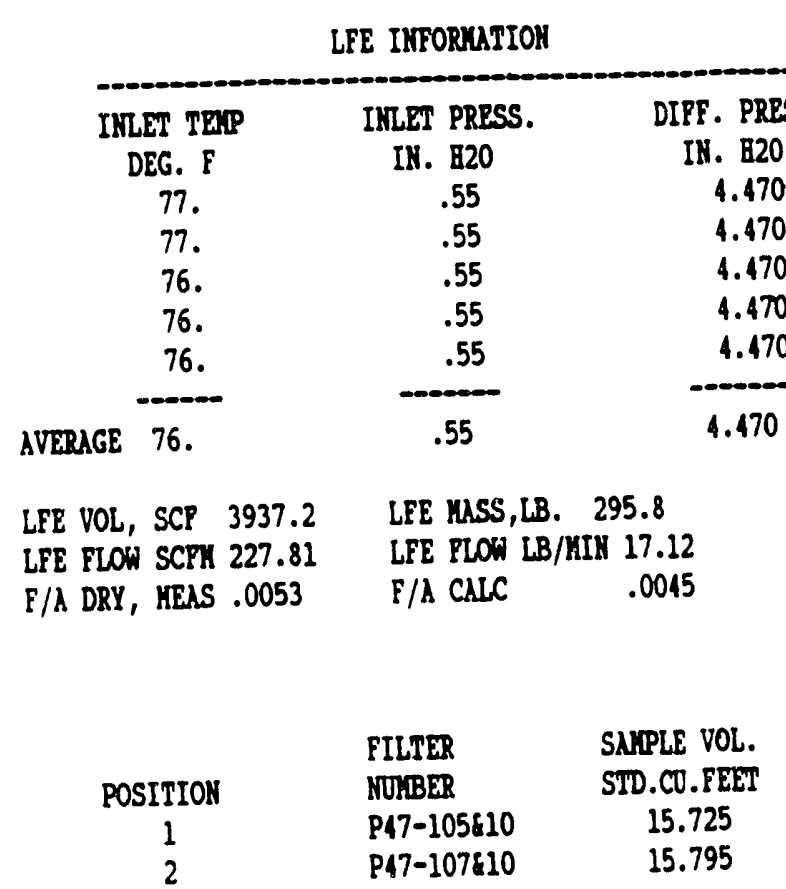

BAROIETER 29.11 IN.BG.

BUIIDITY 36.66 GRAINS B20/LB. AIR LFE 10. 15 ENG. LFE NO. 0 SARPLE TIKE 47m 17.28 MIN. MEAS. EXH. PLON,LB/KIM 17.12 AIR FLON, LB/KIN 17.03

\begin{tabular}{cc} 
BLONER IMTORUATIOH \\
\hline IMLET PRESS. & DIFP. PRESS. \\
IN. B20 & II. B20 \\
10.00 & 14.00 \\
10.00 & 14.00 \\
10.00 & 14.00 \\
10.00 & 14.00 \\
10.00 & 14.00 \\
\hline 10.00 & -14.00
\end{tabular}

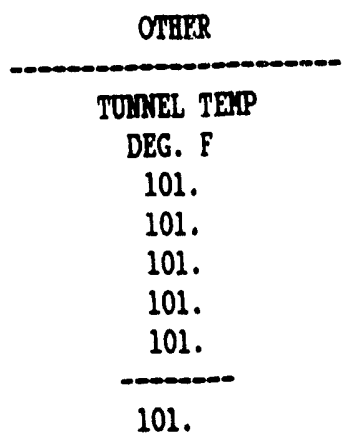

BLONERR + 47m VOL, SCP 4068.4 BLONER + 47w FLON SCYI 235.39 F/A PCT, IEAS -16.0

FILTER DATA

$\begin{array}{ccc}\text { AVG. SAMPLE SYS. } & \text { WEIGHT GAIH } & \text { MG/SCP } \\ \text { TEIP DEG P } & \text { MILLIGRAHS } & \text { OF SAYPLE } \\ 57 . & 2.811 & .179 \\ 57 . & 2.766 & .175\end{array}$

PARTICULATE RESOLTS

AVG. SAPLE 2ORE TEAP, DEG F 101.

DILOTIOA PACTOR 31.019

SPLIT EXHAOST VOL,SCP (SCA), LB/KIH

$131.2(3.71), \quad .57$

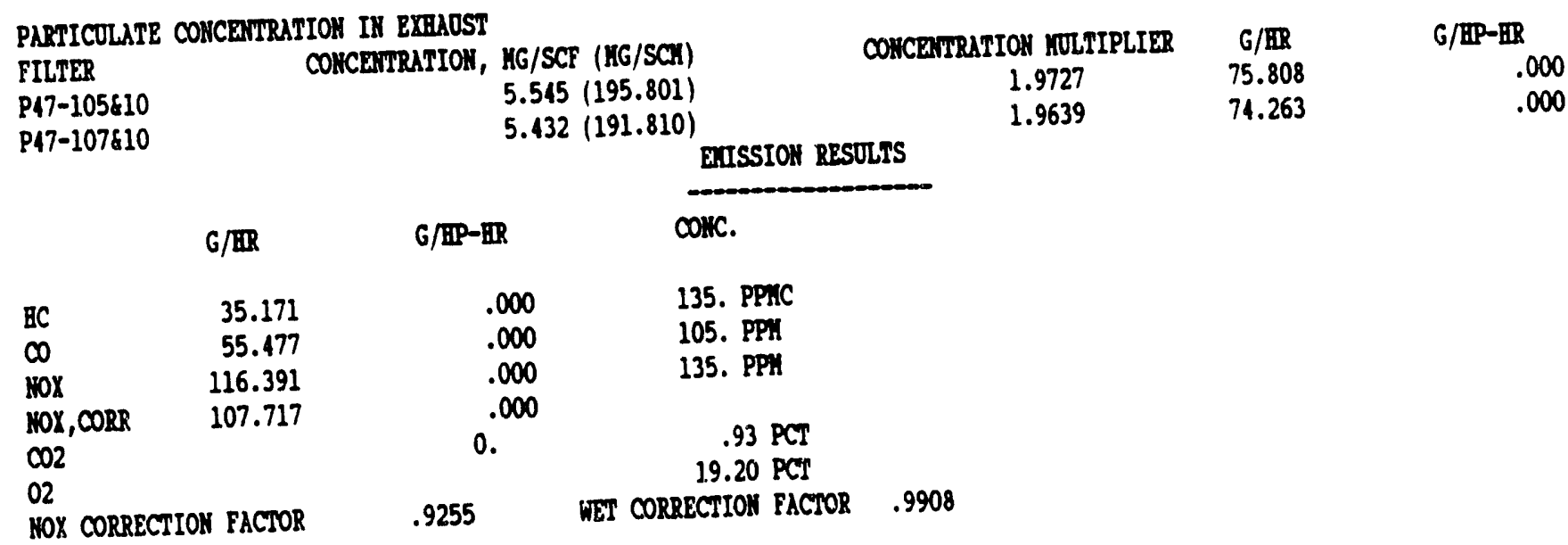


GASEOOS and/Or PARIICULATE EISSSIOAS

SOTCH 1

PROJECT 03-2681-809

PROGRAY = EMD16B

TEST $10 . \quad$ RON 10.8

MIGIYE HODEL EID 2-567B

TRST CELL LOCO LAB

\& ECR 1.656

BLOWER COUITS 40797.

OBS. PONTER, BRP 8.1
TEST DATE $2 / 26 / 91$

DIESEL CONL LIQD

TOSTRL 10. 26

SAIPLE TIIE $20 \times 20 \quad .00 \mathrm{KIN}$.

BLONTRR TEIP. DEG. E 65. FUEL FLON,LB/LIK
.13
BAPOILETER 29.40 IH.EG.

BOIIDITY 40.83 GRAIIS B20/LB. AIR

LFE 10. 15 EIG. LFE 10. 0

SAIPLE TISE 47m 26.98 BIII.

YERAS. EXH. PLON,LB/KIM 16.80

AIR FLOM, IB/KIII
16.67

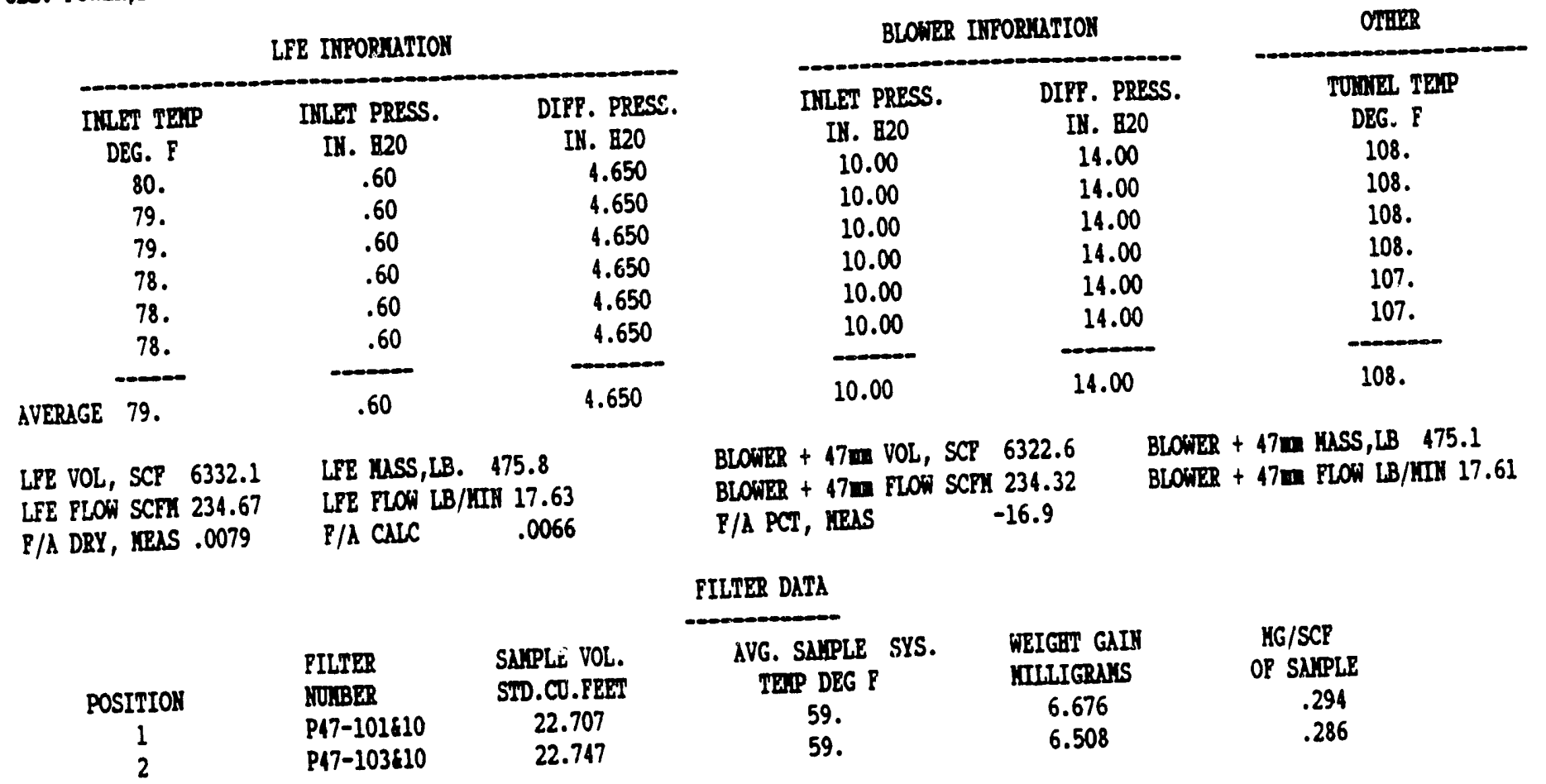

PARICOLATE RESULTS

AVG. SAMPLE ZOTR TEIP, DRG P 108. DILUTIOA PACTOR ttkt

SPLIT EXHAOST VOL,SCF (SCA), LB/IIIH

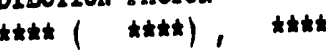

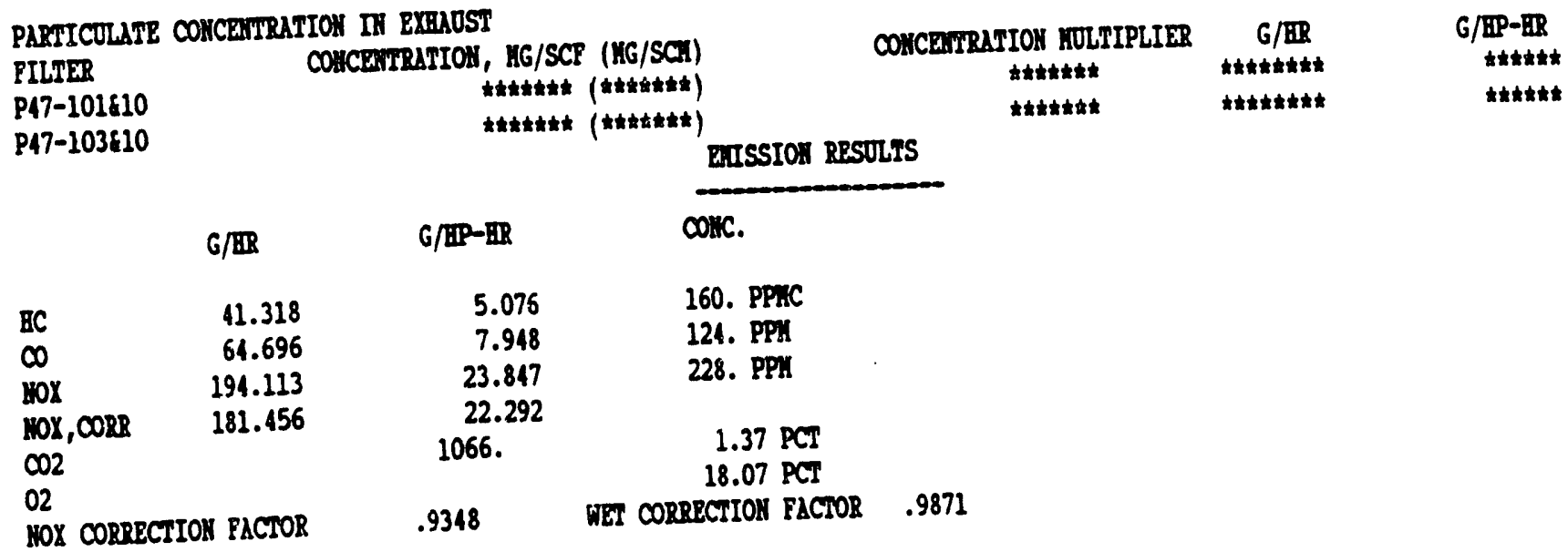


GASEOOS and/Or PARTICOLATE EIISSIOHS

HOTCH 2

PROJECT 03-2681-809

TRST DATE 2/26/91

DIESEL COAL LIQO

TOBAER 10.26

SAMPLE TIME $20 \times 20 \quad .00 \mathrm{KIN}$.

BLOFIR TEIP. DEG. F 68.

FOEL FLON,LB/IIIY

.20

BLOWER COCITSS 41690.

OBS. PONER, BEP 19.0

PROGRAM $=$ END16B

BAPONETER 29.40 IH.HG.

BU:IDITY 32.58 GRUIHS B20/LB. AIR

LFE 10. 15 EIG. LPE HO. 0

SAYPLE TIYE 47m $27.58 \mathrm{MIN}$.

IERS. EXH. FLON,LB/KII 22.13

AIR FLON, LB/HIH 21.93

\begin{tabular}{ccc}
\multicolumn{3}{c}{ LFE INFORUATIOH } \\
\hline IILET TEIP & INLET PRESS. & DIPP. PRESS. \\
DEG. F & IN. B20 & IN. 120 \\
82. & .50 & 4.250 \\
82. & .51 & 4.270 \\
81. & .51 & 4.270 \\
81. & .52 & 4.270 \\
81. & .52 & 4.270 \\
81. & .55 & 4.270 \\
81. & .55 & 4.270 \\
\hline.- .52 & 4.267
\end{tabular}

AVERAGE 81.

LFE VOL, SCP 5914.5

LFE FLOW SCFM 214.42

F/A DRY, KEAS .0092

LFE MASS,LB. 444.4

LFE FLON LB/KII 16.11

F/A CALC

.0080

\begin{tabular}{cc} 
BLOKIRR ITFORMATIOH \\
\hline IRLET PRRSS. & DIFP. PRESS. \\
IN. B20 & IN. B20 \\
10.00 & 14.00 \\
10.00 & 14.00 \\
10.00 & 14.00 \\
10.00 & 14.00 \\
10.00 & 14.00 \\
10.00 & 14.00 \\
10.00 & 14.00 \\
\hline 10.00 & -14.00
\end{tabular}

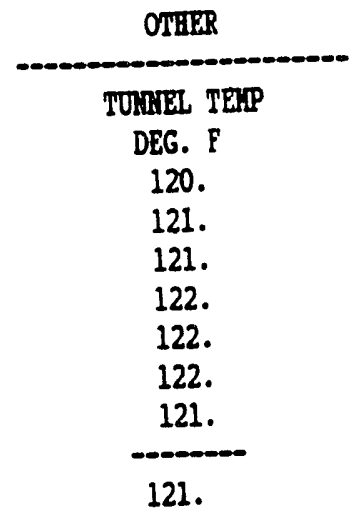

BLOWIR + 47m KLSS,LB 482.6

BLOWIR + 47m FLON LB/KIN 17.50
BLOWER + 47m VOL, SCF 6422.9 BLOFIR + 47m FLON SCPM 232.85 P/A PCT, YERS

$-13.6$

PILTER DATA

$\begin{array}{clc} & & \\ \text { FILTER } & \text { SAYPLE VOL. } \\ \text { POSITION } & \text { NULBER } & \text { STD.CD. FEET } \\ 1 & \text { P47-97\&98 } & 23.124 \\ 2 & \text { P47-996100 } & 23.263\end{array}$

AVG. SAIPLE SYS. TEMP DEG ?

61.

61.

WEIGHT GAIN
MILLIGRAIS
6.306
2.611

MG/SCP

OF SAMPLE .273 .112

PARICULATE RPSULTS

AVG. SAYPLE ZOIR TEIP, DEG P 121. SPLIT EXHAOST VOL,SCF (SCI), LB/KII
DILOTIOA FACTOR 12.635

$508.4(14.40), 1.38$

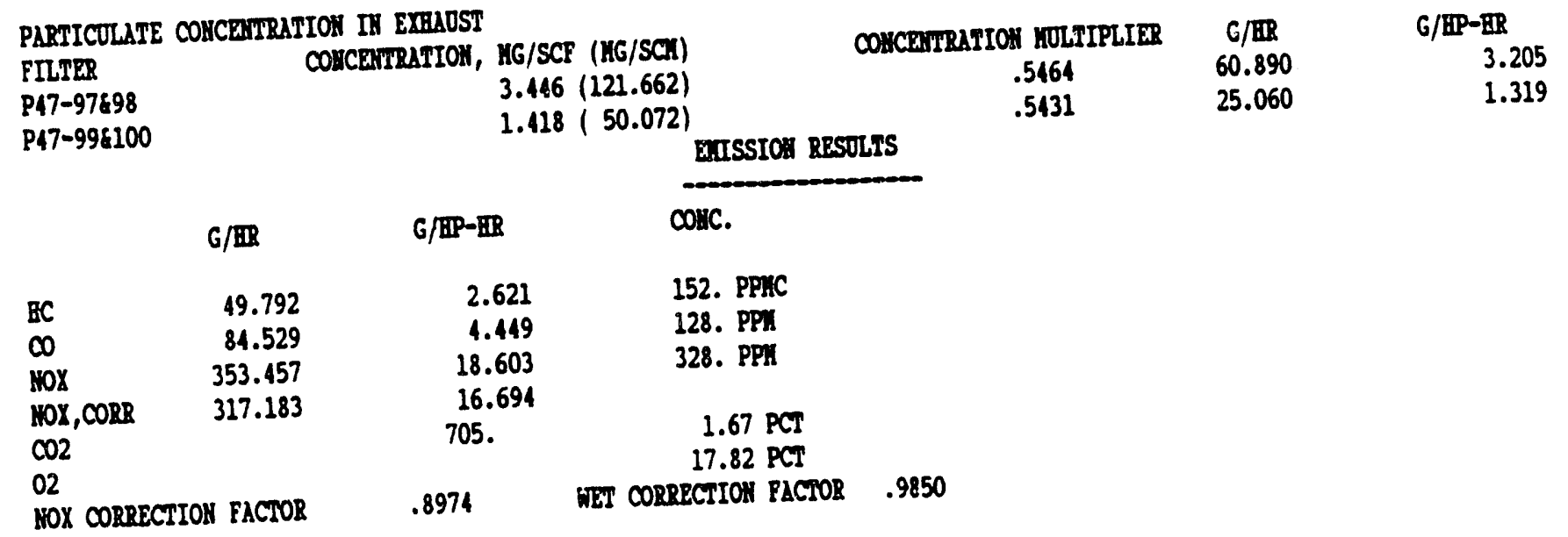

$$
\text { C. }-4
$$


GASEOOS and/Or PARTICULATE EIISSIOHS

HOTCR 3

PROJECT 03-2681-809

PROCRAY = ED16B

TEST NO. ROH NO. 6

EIGINE HODEL EID 2-567B

TEST CELL LOCO LAB

BCR 1.656

BLONER COONTS 20970.

OBS. POFIER,BIP 45.7
TEST DATE 2/26/91

DIESEL COAL LIQO

TUNEL NO. 26

SAIPLE TIJE $20 \times 20 \quad .00$ KIN.

BLONER TEIP. DEG. F 68.

FORL FLOW, LB/MIN
.35

LPE IHPORUATION

\begin{tabular}{ccc} 
IILET TERP & IRLET PRESS. & DIPP. PRESS. \\
DEG. E & IN. H20 & IH. H20 \\
80. & .55 & 4.050 \\
81. & .55 & 4.050 \\
81. & .55 & 4.050 \\
81. & .55 & 4.050 \\
\hdashline 81. & -.55 & -.050
\end{tabular}

LFE VOL, SCF 2834.0

LFE FLOW SCFY 204.32

F/A DRY, IERS .0125

LPE MASS, LB. 212.9

LFE ILON LB/KIN 15.35

F/A CALC
BAROIETER 29.40 IN.BG.

HUITDITY 27.65 GRAINS B20/LB. AIR

LPE NO. 15 ENG. LPE HO. 0

SAMPLE TIYR 47m 13.87 MIH.

IRAS. EXI. FLON,LB/LIY 28.58

AIR PLON,LB/KIM

28.23

\begin{tabular}{|c|c|c|c|c|c|}
\hline \multirow[b]{3}{*}{ POSITION } & \multirow{3}{*}{$\begin{array}{l}\text { FILTER } \\
\text { NOJBER }\end{array}$} & \multirow[b]{2}{*}{$\begin{array}{l}\text { SAIPLE VOL. } \\
\text { STD.CD.PEET }\end{array}$} & \multicolumn{3}{|l|}{ FILTER DATA } \\
\hline & & & $\begin{array}{l}\text { AVG. SAIPLE SYS. } \\
\text { TEIP DEG P }\end{array}$ & $\begin{array}{l}\text { WEIGHT GAIN } \\
\text { KILLIGRAYS }\end{array}$ & $\begin{array}{l}\text { MG/SCP } \\
\text { OP SAIPLE }\end{array}$ \\
\hline & & 11.845 & 65. & 2.879 & .243 \\
\hline 1 & $\begin{array}{l}\text { P47-93\&94 } \\
\text { P47-95\&96 }\end{array}$ & 11.815 & 65. & 2.774 & .235 \\
\hline
\end{tabular}

PARTICOLATE RESULTS

AVG. SAIPLE 2ONE TETP, DEG P 124. DLLOTION FACTOR 8.136

BLOWER + 47W VOL, SCP 3231.1 BLONER $+47 \mathrm{~m}$ FLON SCFM 232.96

F/A PCT, YIRAS -11.7

\begin{tabular}{cc} 
BLONER INPORUATIOH \\
\hline INLET PRESS. & DIPF. PRESS. \\
IK. B20 & IH. E20 \\
10.00 & 14.00 \\
10.00 & 14.00 \\
10.00 & 14.00 \\
10.00 & 14.00 \\
\hdashline 10.00 & -14.00
\end{tabular}

OTHER

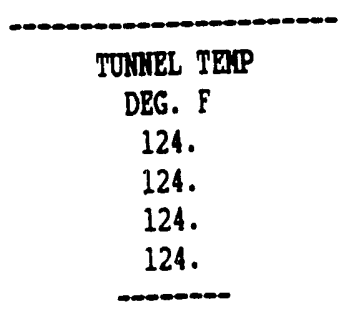

124.
BLONER + 47w MASS, LB 242.8

BLONER + 47m FLON LB/HIN 17.50

SPLIT EXHAOST VOL,SCF (SCH), LB/MTH 397.1 ( 11.25), 2.15

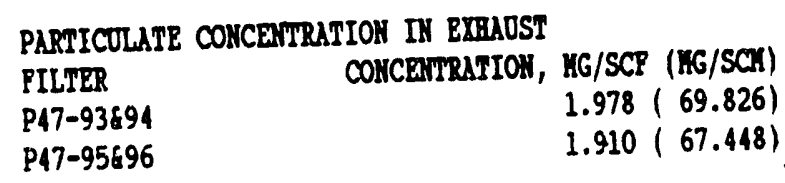

EIISSIOH RESULTS

$\begin{array}{ccc}\text { COICEMTRATIOH IULTIPLIER } & \text { G/HR } & \text { G/HP-ER } \\ .6869 & 45.133 & .988 \\ .6886 & 43.596 & .954\end{array}$

coric.

G/HR

G/BP-ER

$\begin{array}{ll}1.358 & \text { 150. PPHC } \\ 1.427 & 79 . \text { PPH }\end{array}$

13.987 472. PPII

$\begin{array}{ll}\mathrm{BC} & 65.215\end{array}$

110X $\quad 639.049$

12.540

MOX,CORR $\quad 572.945 \quad 516$.

$\mathrm{CO}_{2}$

516

$2.34 \mathrm{PCT}$

$17.06 \mathrm{PCT}$

HOX CORRECTION FACTOR

.8966 WET CORRECTION FACTOR .9798 


\section{GASEOOS and/Or PARTICOLATE EISSSIOHS \\ NOTCH 4 \\ PROJECT 03-2681-809}

TEST HO. ROT 10. 5

EHGINE YODRL END 2-567B

TRST CELL LOCO LAB

HCR $\quad 1.656$

BLONER CODIIS 18634.

OBS. PONER,BLP 76.0

\begin{tabular}{cc} 
& LFE IMPORULTIO \\
\hline INLET TETP & INLET PRESS \\
DEG. F & IN. B20 \\
81. & .59 \\
82. & .60 \\
82. & .60 \\
82. & .60 \\
\hline. .0 & .60
\end{tabular}

TRST DATE $2 / 26 / 91$

DIESEL COAL LIOO

TOLISEL HO. 26

SAIPLE TIIE $20 \times 20 \quad .00 \mathrm{kIH}$.

BLOFER TETP. DBG. F 68.

POEL FLOH,LB/KIY

AVERAGE 82

LPE VOL, SCF 2447.9

LFE FLON SCFI 198.51

F/A DRY, ITHS .0158

LFE MASS,LB. 183.9

LFE PLOH LB/IIIN 14.92

F/A CALC

.0140

DIPP. PRESS.

IK. 820

3.900

3.900

4.000

3.980

3.945
68. .54

$$
\text { PROGRAY = EID16B }
$$

BAROIETER 29.39 IH.BG.

BOIIDITY 24.39 GRAINS B20/LB. AIR

LFE 10. 15 EIG. LPE NO. 0

SAMPLE TISE $47 \mathrm{~m} 12.33$ KIH.

MEAS. EXH. FLON,LB/KIK 34.79

AIR FLON, LB/KIM $\quad 34.25$

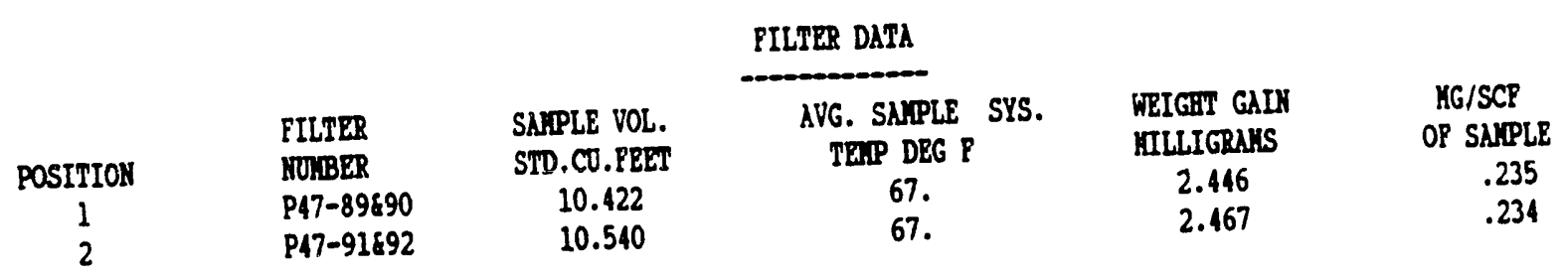

PARTICULATE RESOLTS

AVG. SAYPLE ZONR TEAP, DEG F 123. DILOTION PACTOR 6.800

BLONER + 47m VOL, SCP $2869.9 \quad$ BLOWER + 47m MASS, LB 215.6

BLONER + 47w FLON SCFH 232.73 BLONER + 47W FLON LB/KIN 17.49

$\mathrm{P} / \mathrm{A}$ PCT, KRAS -11.3

DIPP. PRRSS.

II. $\mathrm{B} 20$

14.00

14.00

14.00

14.00

14.00

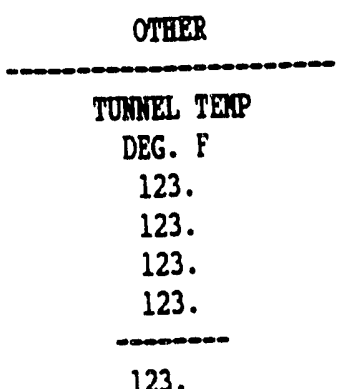

123. SPLIT EXHAOST VOL,SCF (SCA), LB/LIT $122.0(11.95), 2.57$

PARTICULATE CORCRTIRATION II EXthOST

FILTER

P47-89\$90

P47-91\&92

$\begin{array}{lr} & \text { G/HR } \\ & \\ \text { BC } & 72.697 \\ \text { O } & 64.024 \\ \text { MOX } & 925.572 \\ \text { HOX, CORR } & 824.585 \\ \text { CO2 } & \\ \text { O2 } & \\ \text { HOX CORRECTION FACTOR }\end{array}$

MOX CORRECTION FACTOR
COMCEMTRATIOH, MG/SCF (IG/SCH)

$1.596(56.355)$

$1.592(56.201)$
COMCRITRATIOA MULTIPLIER

.6525

.6452
G/IR
44.336
44.215

AIISSIOH RESULTS
G/EP-ER

.583

.582

conc.

$$
\text { G/EP-ER }
$$

.957

.842

12.179

10.850

476.

.8909
145. PPiC

64. PPII

567. PPH

\section{$2.98 \mathrm{PCT}$}

16.18 PCT

WET CORRECTIOH FACTOR .9749 
GASEOOS and/Or PARTICULATE WISSIOHS

NOTCH 5

PROJECT 03-2681-809

PROGRAY = EDI6B

TEST NO. RON NO. 4

ENGIKE MODEL EID 2-567B

TEST CELL LOCO LAB

BCR 1.656

BLOFER COUNTS 32237.

OBS. PONER, BHP 113.0
TEST DATE 2/26/91

DIESEL COLL LIQD

TOWAEL NO. 26

SAYPLE TIYE $20 \times 20 \quad .00$ KIN.

BLOFTR TEIP. DEG. F 68.

FOEL FLOW, LB/KIH
68.
BAROKLETER 29.39 IN.BG.

BIUIDITY 22.80 GRAIIS B20/LB. AIR

LFE NO. 15 ENG. LFE 10.0

SAIPLE TIYR 47m 21.33 KIH.

NEAS. EXE. FLON, LB/KTH 40.96

AIR FLON, LB/KIN $\quad 40.20$

\begin{tabular}{|c|c|c|}
\hline \multicolumn{3}{|c|}{ LFE IKFORHATIOH } \\
\hline IRLET TEIP & IKLET PRPSS. & DIFT. PRESS. \\
\hline DEG. $F$ & IN. 820 & IN. $\mathrm{B} 20$ \\
\hline 84 & .63 & 3.900 \\
\hline 83. & .63 & 3.920 \\
\hline 83. & .63 & 3.900 \\
\hline 83. & .63 & 3.900 \\
\hline 83. & .63 & 3.900 \\
\hline 83. & .63 & 3.900 \\
\hline AGE 83. & .63 & 3.903 \\
\hline
\end{tabular}

LFE VOL, SCP 4172.3

LFE PLOW SCFY 195.58

F/A DRY, IIRAS .0190
LFE MASS,LB. 313.5

LFE PLON LB/KIN 14.70

P/A CALC $\quad .0182$

\begin{tabular}{cc} 
BLONER INTORUATION \\
\hline IRLET PRRSS. & DIFF. PRESS. \\
IN. B20 & IN. B20 \\
10.00 & 14.00 \\
10.00 & 14.00 \\
10.00 & 14.00 \\
10.00 & 14.00 \\
10.00 & 14.00 \\
10.00 & 14.00 \\
\hdashline-0.00 & 14.00
\end{tabular}

BLOKER + 47w VOL, SCP 4964.3

BLOWER + 47w FLOW SCFI 232.70

P/A PCT, MEAS

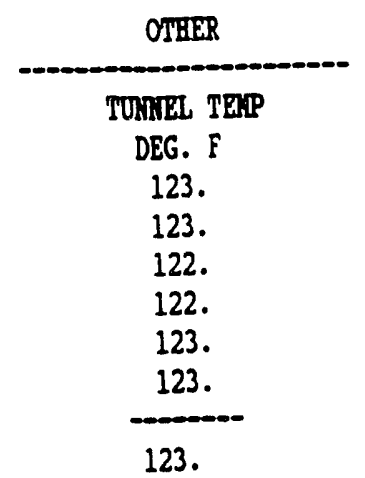

BLONTER + 47m MLSS,LB 373.0 BLOWER + 47m PLOW LB/KIN 17.49

FILTER DATA

\begin{tabular}{|c|c|c|c|c|c|}
\hline \multirow[b]{3}{*}{ POSITION } & & & $-\infty$, & & \\
\hline & FILTER & SANPLE VOL. & AVG. SAYPLE SYS. & $\begin{array}{l}\text { WEIGHT GAIK } \\
\text { MILLIGRAYS }\end{array}$ & $\begin{array}{l}\text { HG/SCP } \\
\text { OP SAYPLE }\end{array}$ \\
\hline & IOLBER & STD.CO.FEET & $\begin{array}{c}\text { TEIP DEG } \mathrm{r} \\
67 .\end{array}$ & 3.532 & .199 \\
\hline 1 & $\begin{array}{l}P 47-85 \& 86 \\
P 47-87 \& 88\end{array}$ & $\begin{array}{l}17.715 \\
17.764\end{array}$ & $\begin{array}{l}67 . \\
67 .\end{array}$ & 3.436 & .193 \\
\hline
\end{tabular}

PARTICULATE RESULTS

AVG. SAYPLE 2ONE TEIP, DEG F 123. DILOTION FACTOR 6.269

SPLIT EXHAOST VOL,SCF (SCA), LB/KIIH 791.9 ( 22.43), 2.79

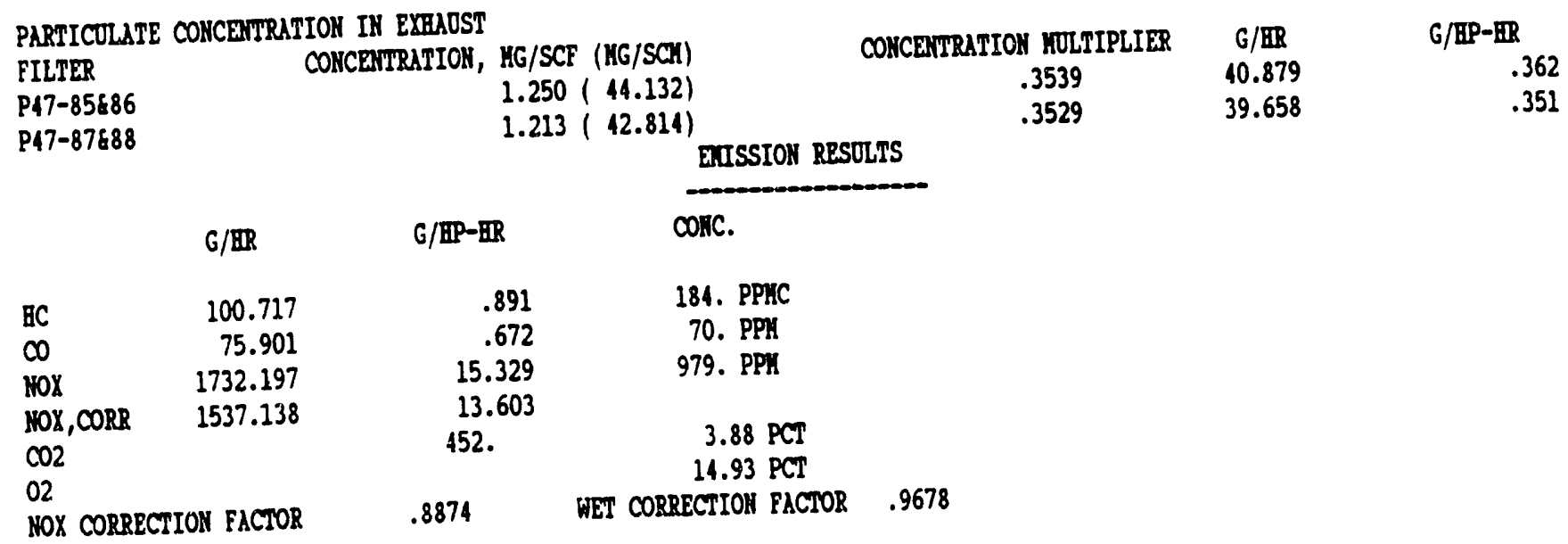


GASEOOS and/Or PARTICULATE EIISSIOHS

NOTCH 6

PROJECT 03-2681-809

TRST DATE $2 / 26 / 91$

DIESEL COAL LIQO

TOHARL NO. 26

SAIPLE TIYE $20 \times 20 \quad .00 \mathrm{kTM}$.

BLOFER TETP. DEG. F 67.

FORL FLON,LB/YTH $\quad 1.00$
PROGRAY = ED16B

BAROIETER 29.43 IH.EG.

BOUIDITY 25.10 GRAIHS B20/LB. AIR

IFE 110. 15 EHG. LPE 10. 0

SAFPLE TISE 47m $30.00 \mathrm{kIn}$.

REAS. BXE. FLOW,LB/KIH 48.47

AIR FLOW, LB/MIIK

47.47

OBS. POWER,BRP 149.0

LPE IITORMATION

\begin{tabular}{|c|c|c|}
\hline IIREET TRAP & IILLET PRESS. & DIPP. PRESS. \\
\hline $\begin{array}{c}\text { DEG. F } \\
83 .\end{array}$ & IN. B20 & IN. E20 \\
\hline $\begin{array}{l}83 . \\
83 .\end{array}$ & .63 & 3.900 \\
\hline $\begin{array}{l}83 . \\
83 .\end{array}$ & .65 & 3.930 \\
\hline 83. & .65 & 3.950 \\
\hline 83. & fij & 3.950 \\
\hline 83. & .65 & 4.000 \\
\hline 83. & .65 & 4.000 \\
\hline 83. & .65 & 4.000 \\
\hline 83. & $.6 j$ & 3.961 \\
\hline
\end{tabular}

LFE VOL, SCF 5961.8

LFE PLOW SCTI 198.74

F/A DRY, KRAS .0212
LFE MASS, LB. 448.0

LPE PLON LB/KIN 14.93

$\mathrm{P} / \mathrm{A}$ CALC

.0212

\begin{tabular}{cc}
\multicolumn{2}{c}{ BLOWIR IMPORLATIOH } \\
\hline IIRET PRESS. & DIPP. PRRSS. \\
IH. H20 & IN. E20 \\
10.00 & 14.00 \\
10.00 & 14.00 \\
10.00 & 14.00 \\
10.00 & 14.00 \\
10.00 & 14.00 \\
10.00 & 14.00 \\
10.00 & 14.00 \\
\hline 10.00 & -.20 \\
\hline 14.00
\end{tabular}

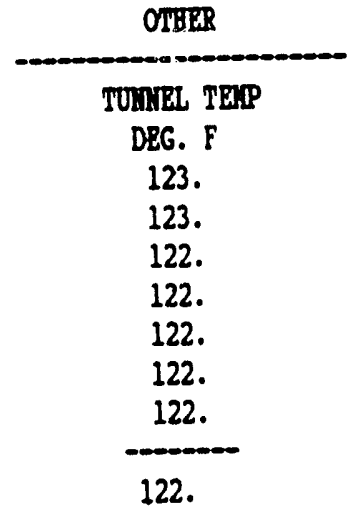

BLOWER + 47m VOL, SCP 7009.2 BLONRR + 47m FLON SCTI 233.65

BLONER + 47m MASS,LB 526.7

I/A PCT, IERS

BLOWER + 47m PLOW LB/IIH 17.56

FILTER DATA

$\begin{array}{clc} & \text { PILTER } & \text { SANPLE VOL. } \\ \text { POSITION } & \text { NOIBER } & \text { STD.CD.FEET } \\ 1 & \text { P47-81682 } & 25.102 \\ 2 & \text { P47-83684 } & 25.092\end{array}$

AVG. SAYPLE SYS.

TRIP DEG $F$

66.
.0

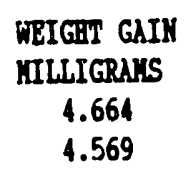

MG/SCP

OF SAIPLE

.186

.182

PARPICOLATE RESOLTS

AVG. SAIPLE ZONE TEP, DEG P 122 . DILDTIOH PACTOR 6.692

SPLIT EXHAOST VOL,SCP (SCH), LB/AIH $1047.4(29.66), 2.62$

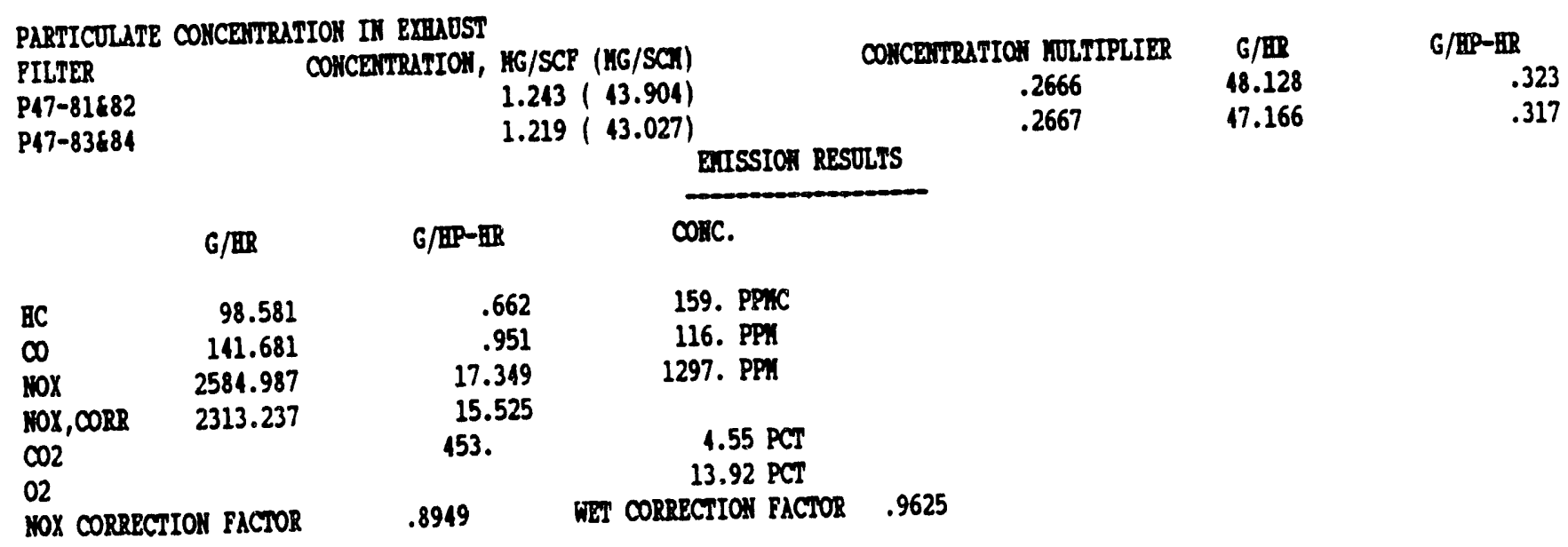


GASEOOS and/Or PARTICULATE EIISSIONS

roTCH 7

PROJECT $03-2681-809 \quad$ PROGRAY $=$ EIDI6B

TEST NO. ROH HO. 2 TEST DATE $2 / 26 / 91$

ESGIKE HODEL ERD 2-567B

TEST CELL LOCO LAB

BCR 1.656

BLONER COONTS 45271.

OBS. POWER, BAP 179.0
DIESEL COAL LIQO

TOSNEL NO. 26

SAIPLE TIKE $20 \times 20 \quad .00 \mathrm{KIH}$.

BLONER TEAP. DEG. F 70.

FOEL FLON, LB/KIN $\quad 1.23$
BAROIETER 29.47 II.BG.

BOIIDITY 25.84 GRAIHS B20/LB. AIR

LFE HO. 15 EMG. LFE HO. O

SAIPLE TIKE 47m 30.00 KIH.

VRAS. EXH. FLON,LB/XTH 55.23

AIR PLOW, LB/MIN $\quad 54.00$

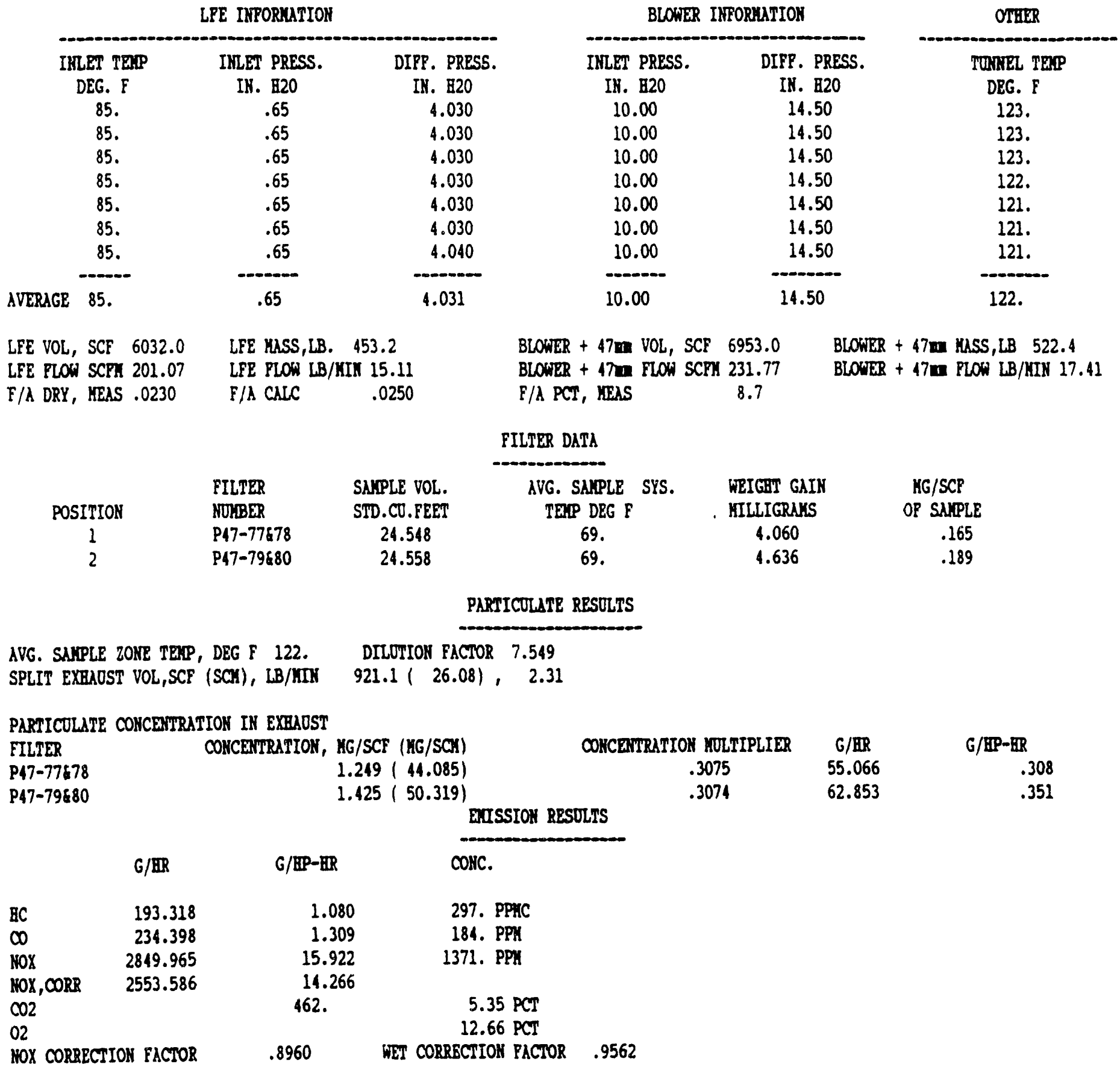


GLSEOOS and/Or PARTICULATE EIISSIONS

HOTCH 8

PROJECT 03-2681-809

TEST DATE 2/26/91

DIESEL CONL LIQO

TONIEL HO. 26

SAYPLE TIIL $20 \times 20 \quad .00$ YIH.

BLONTR TETP. DEG. F 66.

FOEL FLON,LB/KIY $\quad 1.48$

1.48

PROCRAY $=$ EMD16B

BARONETER 29.49 $\mathrm{X} . \mathrm{BG}$.

BOIIDITY 35.93 GRAIHS B20/LB. AIR

LPE 10. 15 BIG. LPE 110.0

SAMPLE TIME 47m 30.00 KIH. REAS. EXH. PLOW,LB/KIH 62.06 AIR PLOW, LB/KIM
60.58
BCR 1.656
BLONER COUITS 45375.

OBS. POHER,BRP 206.0

\begin{tabular}{|c|c|c|}
\hline \multicolumn{3}{|c|}{ LPE IYFORYATION } \\
\hline INLET TERP & IRLET PRESS. & DIFF. PRESS. \\
\hline DEG. F & IN. B20 & IK. 1820 \\
\hline 83. & .65 & 4.100 \\
\hline 83. & .65 & 1.100 \\
\hline 83. & .65 & 4.100 \\
\hline 83. & .65 & 4.100 \\
\hline 83. & .65 & 4.100 \\
\hline 83. & .65 & 4.100 \\
\hline 83. & .65 & 4.100 \\
\hline 83 & .65 & 4.100 \\
\hline
\end{tabular}

LFE VOL, SCP 6173.8

LPE FLOW SCFI 205.81

F/A. DRY, KIEAS .0246
LFE MASS,LB. $\quad 463.9$

LFE PLON LB/KIIH 15.46

F/A CALC
.0294
BLONER TIPORYATION

IRLET PRESS.
IN. B20
10.00
10.00
10.00
10.00
10.00
10.00
10.00
10.00

DIPP. PRESS.

III. 120

14.50

14.50

14.50

14.50

14.50

14.50

14.50

14.50

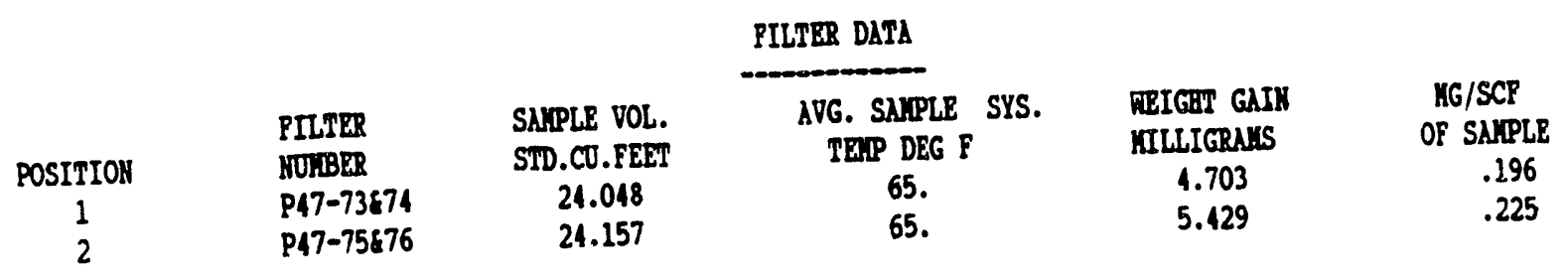

PARTICULATE RESULTS

AVG. SAIPLE 2OKE TEIP, DEG F 120 . DILOTIOH PACTOR 8.218

BLONER + 47W VOL, SCP 7029.1 BLOWTR + 47m PLOW SCPI 234.32 P/A PCT, YRAS $\quad 19.3$

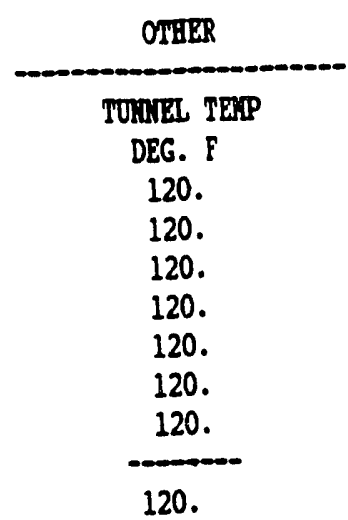

BLONER + 47W WLSS,LB 528.2 BLOWER + 47 FLON LB/KIH 17.61 SPLIT EXHAOST VOL,SCF (SCA), LB/YTI $855.3(24.22), \quad 2.14$

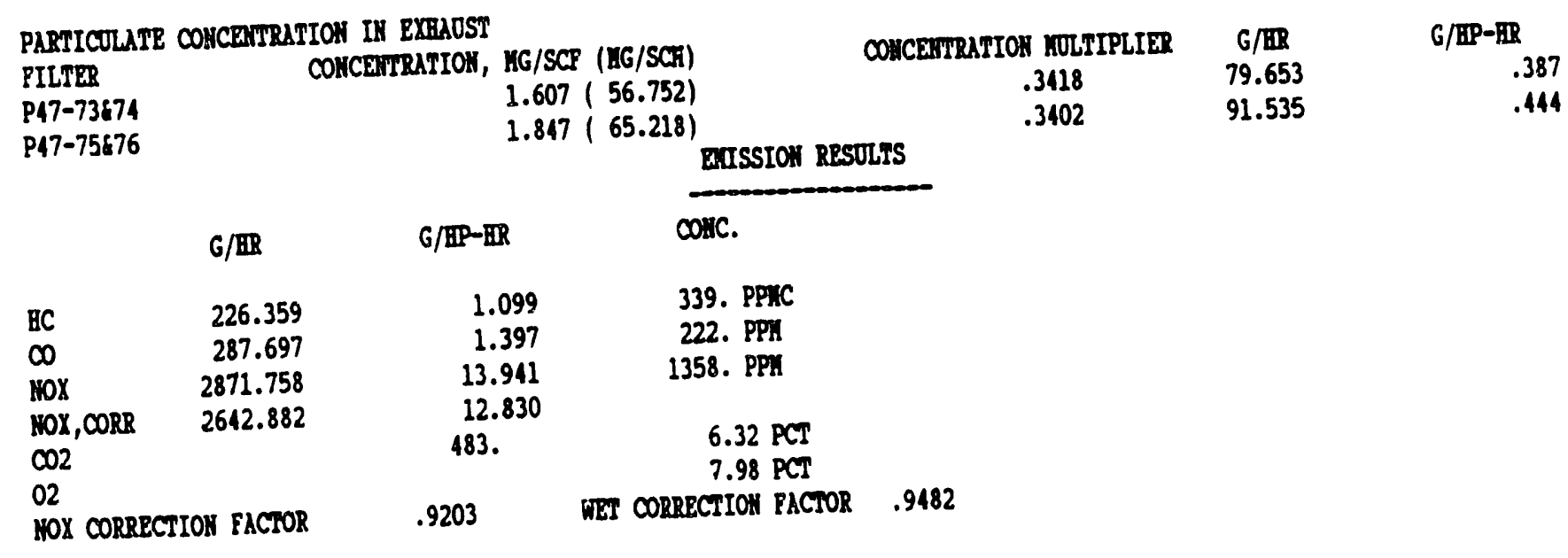


APPENDIX D

D-1 
GASEOOS and/or PARTICULATE EIISSIONS

IDLE
PROJECT 03-2681-809

TEST DATE $2 / 28 / 91$

DIESEL DF-2

TUIATEL 10. 26

SAMPLE TIYE $20 \times 20 \quad .00 \mathrm{KIH}$.

BLONIR TR:P. DEG. F 71.

FOEL FLON,LB/KIK
.09
TEST CRLLL LOC
BCR 1.758

BLONER COUITS 45069.

OBS. POWER,BEP .0

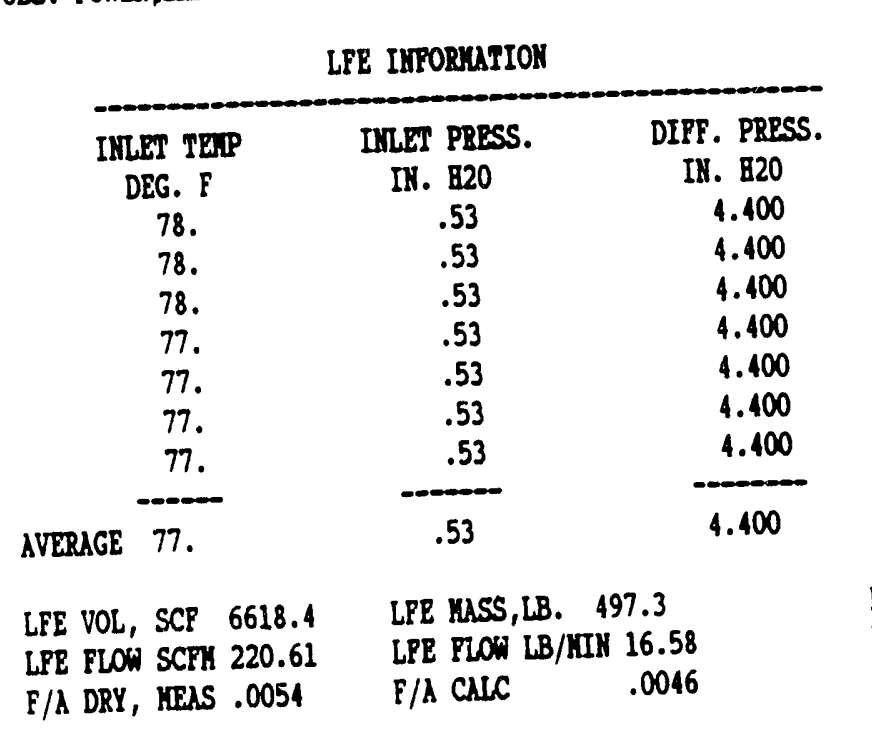

PROGRAN = EDD16B

BAROIETER 29.01 IH.BG.

BOUIDITY 83.92 GRAIIS B20/LB. AIR

LPE 10. 15 ETG. LFE 10. 0

SAIPLE TIYT $47 \mathrm{~m} 30.00$ KIH.

MRAS. BXH. PLON,LB/HIIN 16.94

AIR FLON, LB/KIH

16.85
BLOWER IMFORUATION

\begin{tabular}{cc} 
IRLET PRESS. & DIPP. PRESS. \\
IN. B20 & IN. B20 \\
10.00 & 14.00 \\
10.00 & 14.00 \\
10.00 & 14.00 \\
10.00 & 14.00 \\
10.00 & 14.00 \\
10.00 & 14.00 \\
10.00 & 14.00 \\
\hline 10.00 & -14.00
\end{tabular}

OTHER

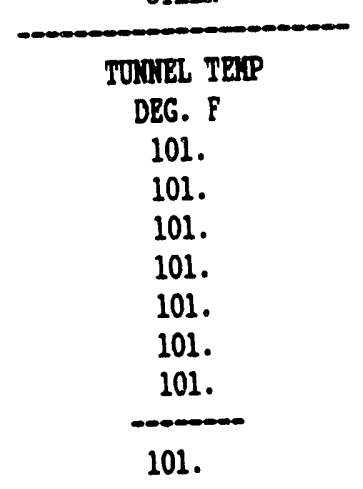

BLONER + 47w VOL, SCP 6796.7 BLONER + 47m ULSS,LB 510.7 BLONTER $+47 \mathrm{~m}$ FLON SCFH 226.56 BLONER $+47 \mathrm{~m}$ PLON LB/YIN 17.02 P/A PCT, ITAS -14.1

\section{FILTER DATA}

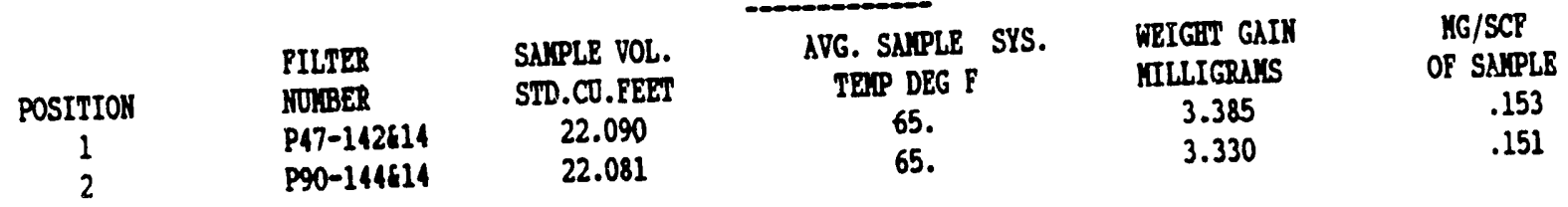

PARICOLATE RESULTS

AVG. SAYPLE 2OKE TERP, DEG F 101. DILOTIOI PACTOR 38.118

SPLIT EXALOST VOL,SCF (SCH), LB/:III $178.3(5.05), \quad .45$

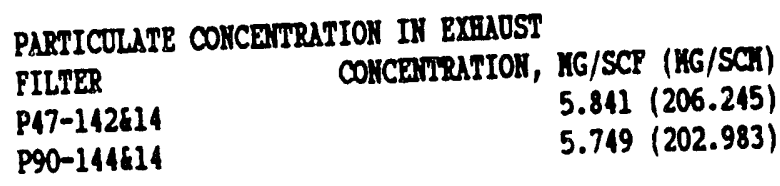

\section{COBCEITRATIOA RULTIPLIER}

1.7255

1.7263
G/ER

79.009

77.760
G/EP-IR .000 .000

\section{E.ISSIOA RESULIS}

\begin{tabular}{|c|c|c|c|c|}
\hline & G/ER & 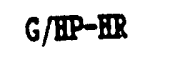 & corrc. & \\
\hline Ac & 29.001 & .000 & 115. PPHC & \\
\hline$\infty$ & 41.799 & .000 & 82. PPH & \\
\hline $\begin{array}{l}\text { MOX } \\
\text { MOX, CORR }\end{array}$ & 96.748 & .000 & i17. PPH & \\
\hline $\begin{array}{l}\mathrm{NOX}, \mathrm{CORR} \\
\mathrm{CO} 2\end{array}$ & 108.285 & 0.000 & $\begin{array}{r}.96 \mathrm{PCT} \\
18.95 \mathrm{PCT}\end{array}$ & \\
\hline O2 & FACTOR & 1.1193 & WET CORRECTIOH FACTOR & .9882 \\
\hline
\end{tabular}


GASBOOS and/Or PARTICOLATE BuISSIOAS

NOTCR 1

PROJECT 03-2681-809

PROGRAY $=$ DIDI6B

TEST 10.

ROI 10. 8

TEST DATE 2/28/91

ENGINE YODEL EDD 2-567B

TEST CELL LOCO LAB

HCR 1.758

BLOWER COUITS 23590.

OBS. PONER, BAP 8.4

DIESEL DF -2

TUATRL 10. 26

SAYPLE TIEE $20 \times 20 \quad .00$ VIH.

BLONER TEEP. DEG. F 72.

FUEL FLON, LB/KIN

.13

BAROHETER' 28.91 IH.BG.

HOYIDITY 89.86 GRAIHS B20/LB. AIR

LFE HO. 15 ERG. LPE MO. 0

SANPLE TINE 47m 15.69 IIIN.

IRRIS. EXR. FLOW,LB/KIYY 16.71

AIR FLON,LB/KIII

16.58

LPE IRTORYATION

\begin{tabular}{ccc} 
IMLET TEIP & IIRET PRESS. & DIPF. PRRSS. \\
DEG. F & IN. B20 & II. B20 \\
82. & .55 & 4.700 \\
82. & .55 & 4.700 \\
82. & .55 & 4.700 \\
80. & .55 & 4.700 \\
\hdashline 82. &. .55 & 4.700
\end{tabular}

LFE VOL, SCP 3624.2

LFE FLON SCFK 230.99

LFE UASS,LB. 272.3

LFE PLON LB/HIII 17.36

P/A CALC

.0070

\begin{tabular}{cc}
\multicolumn{2}{c}{ BLOFER INTORUATIOH } \\
\hline IKLET PRESS. & DIPF. PRESS. \\
IN. B20 & IH. B20 \\
10.00 & 14.00 \\
10.00 & 14.00 \\
10.00 & 14.00 \\
10.00 & 14.00 \\
\hline 10.00 & 14.00
\end{tabular}

OTHER
TUITEL TEIP
DEG. E
111.
112.
111.
110.
111.

BLONRR + 47m VOL, SCP 3538.2 BLONER + 47m MSS,LB 265.9

BLONER + 47m FLON SCFY 225.51 BLOWER + 47m FLON LB/YII 16.94

F/A PCT, IRAS $\quad-10.0$

FILTER DATA

\begin{tabular}{|c|c|c|c|c|c|}
\hline & $\begin{array}{l}\text { PILTER } \\
\text { NUTBER }\end{array}$ & SANPLE VOL. & $\begin{array}{l}\text { AVG. SALPLP SYS. } \\
\text { TRIP DEG F }\end{array}$ & $\begin{array}{l}\text { WEIGEI GAIY } \\
\text { MILIIGRAS }\end{array}$ & $\begin{array}{l}\text { KG/SCF } \\
\text { OP SAIPLE }\end{array}$ \\
\hline $\begin{array}{c}\text { POSITION } \\
1\end{array}$ & NOIBER & STD.CD.PEET & $\begin{array}{l}\text { TEIP DEG I } \\
68 .\end{array}$ & 4.228 & .360 \\
\hline $\begin{array}{l}1 \\
2\end{array}$ & $\begin{array}{l}P\{7-138\{13 \\
P 90-140 \$ 14\end{array}$ & $\begin{array}{l}11.740 \\
11.701\end{array}$ & 68. & 4.166 & .356 \\
\hline
\end{tabular}

PARTICULATE RESULTS

AVG. SANPLE ZONE TERP, DEG \& 111.

DILOTIOR PACTOR ktktkt

SPLIT EXHAOST VOL,SCF (SCA), LB/YIH

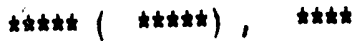

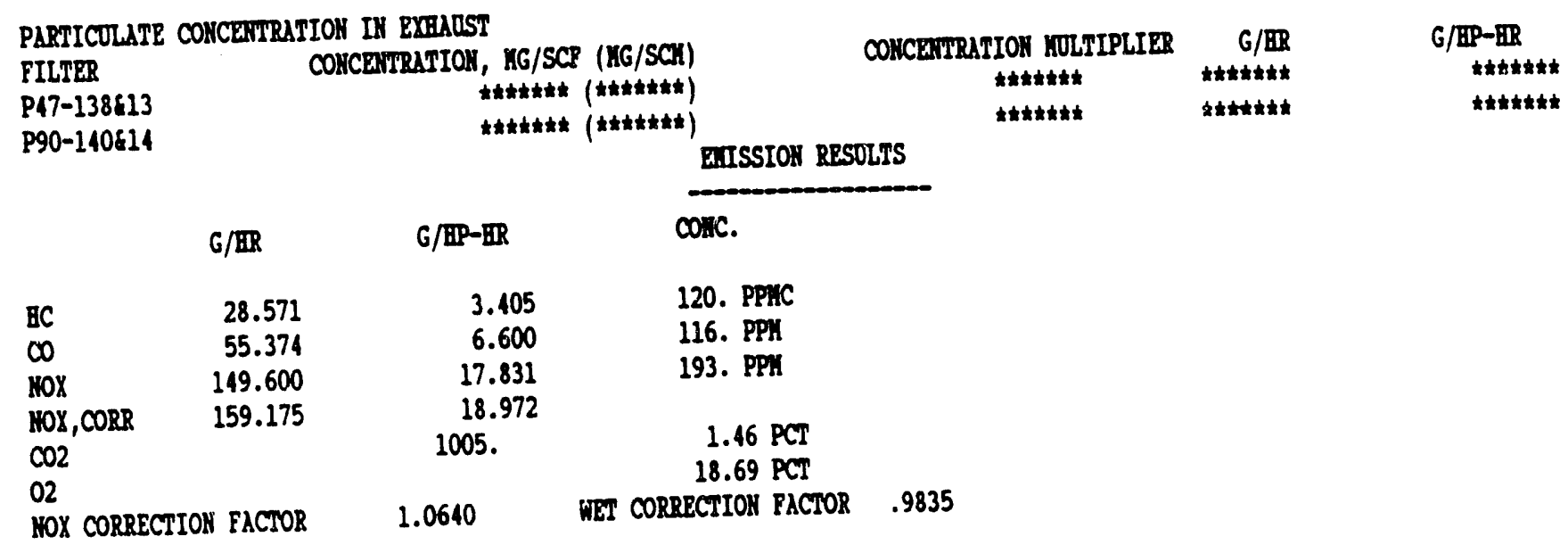


PROJECT 03-2681-809

TEST HO. RON 10.7

ENGINE KODEL EXD 2-567B

TEST CELL LOCO LAB

BCR 1.758

BLONTER COURTS 36081.

OBS. POWER, BRP 19.7
TEST DATE $2 / 28 / 91$

DIESEL DF -2

TOTAR 10. 26

SNIPLE TIKE $20 \times 20 \quad .00$ MIN.

BLONER TERP. DEG. \& 74.

FOEL PLON,LB/KII

.20
PROGRAI = DID16B

BAPOXIETER 29.07 IV.BG.

BUIDDITY 87.16 GRALS B20/LB. AIR

LFE 10. 15 BIG. LPE 10.0

SAYPLE TINE 470 24.00 YIIH. ITRAS. EXH. PLON,LB/KIIS 22.13

AIR FLON,LB/KIH

21.93

\begin{tabular}{|c|c|c|}
\hline \multicolumn{3}{|c|}{ LFE IMFORLTION } \\
\hline $\begin{array}{c}\text { IKLET TEIP } \\
\text { DEG. } P \\
79 . \\
79 . \\
79 . \\
79 . \\
79 . \\
79 .\end{array}$ & $\begin{array}{c}\text { IILET PRESS. } \\
\text { IN. B20 } \\
.50 \\
.50 \\
.50 \\
.50 \\
.50 \\
.50\end{array}$ & $\begin{array}{c}\text { DIFT. PRESS. } \\
\text { IH. B20 } \\
4.290 \\
4.290 \\
4.280 \\
4.290 \\
4.300 \\
4.290\end{array}$ \\
\hline 79. & .50 & 4.290 \\
\hline
\end{tabular}

LFE VOL, SCP 5153.3 LFE FLOW SCFK 214.71 F/A DRY, IEAS .0092

LPE MUSS,LB. 387.2 LFE FLON LB/KIH 16.13 F/A CALC

.0080

\begin{tabular}{cc} 
BLOWER IMPORUATION \\
\hline IILET PRESS. & DIPP. PRESS. \\
IH. B20 & IH. B20 \\
10.00 & 14.00 \\
10.00 & 14.00 \\
10.00 & 14.00 \\
10.00 & 14.00 \\
10.00 & 14.00 \\
10.00 & 14.00 \\
\hline 10.00 & 14.00
\end{tabular}

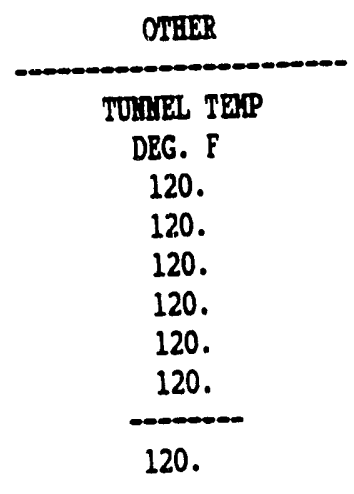

BLONTRR + 47m ILSS,LB 407.4 BLONER + 47E PLON LB/KIH 16.98 BLONER + 47m VOL, SCP 5422.5 BLONRR + 47m FLON SCFI 225.92 F/A PCT, KERAS

$-13.4$

FILTER DATA
AVG. SAIPLE SYS. TEIP DEG ?

67.

67.

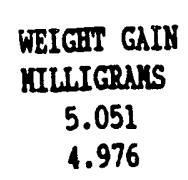

KG/SCF

OP SANPLE .280 .276
PARICULATE RESULIS

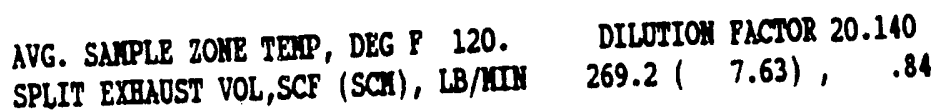

PARTILULATE CONCEATRATION IN EXALOST

FILTER

P47-134\$13

$\mathrm{P} 90-136813$

\begin{tabular}{lr} 
& \multicolumn{1}{c}{ G/ER } \\
BC & 40.811 \\
$\infty$ & 73.537 \\
NOX & 285.514 \\
IOX, CORR & 318.895 \\
CO2 & \\
O2 & \\
NOX CORRECTIOH FACTOR
\end{tabular}

672.
COICEITRUTIOH, MG/SCF (HG/SCI)

$5.646(199.377)$

$5.554(196.098)$
COACEITLATION NOLTIPLIRR
1.1179
1.1161

\section{EIISSION RESULTS}

G/ER

99.780

98.139
G/BP-BR

5.065

4.982 cosic.

G/EP-BR

125. PPAC

3.733 113. PPI

14.493 268. PPH

16.188

$1.67 \mathrm{PCT}$

18.07 PCT

WET CORRECTIOH FACTOR .9819 
GASEOUS and/Or PARTICULATE EUISSIOHS

HOTCH 3

PROJECT 03-2681-809

TEST HO. ROH HO. 6

EMGINE HODEL EXD 2-567B

TEST CELL LOCO LAB

BCR 1.758

BLONER COUITS 45099.

OBS. POWER, BIP 46.0
TRST DATR 2/28/91

DIESEL DP-2

TOWSER 10. 26

SAMPLE TISE $20 \times 20 \quad .00 \mathrm{KIN}$.

BLONER TEIP. DEG. F 74. POEL FLOF, LB/KTI
PROGRAK = EDI6B

BAROMETER 29.07 II. BG.

HOUIDITY 82.07 GRAIIS H20/LB. AIR

LPE NO. 15 ENG. LPE NO. 0

SA.PLE TIKR 47m $30.00 \mathrm{MTH}$.

KRAS. EXA. FLON,LB/KIH 28.22

AIR FLON, LB/III

\begin{tabular}{|c|c|c|}
\hline \multicolumn{3}{|c|}{ LFE IMFORKATIOH } \\
\hline $\begin{array}{l}\text { INLET TEAP } \\
\text { DEG. } \bar{F}\end{array}$ & $\begin{array}{l}\text { IMLET PRESS. } \\
\text { IN. H20 }\end{array}$ & $\begin{array}{l}\text { DIPF. PRRSS. } \\
\text { IN. H } 20\end{array}$ \\
\hline 81. & .55 & 4.100 \\
\hline 81. & .55 & 4.100 \\
\hline 81. & .55 & 4.100 \\
\hline 81. & .55 & 4.100 \\
\hline 81. & .55 & 4.100 \\
\hline 81. & .55 & 4.100 \\
\hline 81. & .55 & 4.100 \\
\hline & .55 & 4.100 \\
\hline
\end{tabular}

LPE VOL, SCP 6128.1

LFE FLOW SCFK 204.25

F/A DRY, MEAS .0127
LFE MASS, LB. 460.5 LFE FLN LB/KIN 15.35 F/A CALC

\begin{tabular}{cc}
\multicolumn{2}{c}{ BLOWIRR IMPORYATIOH } \\
\hline IMLBT PRESS. & DIFF. PRESS. \\
IN. B20 & IN. B20 \\
10.00 & 14.00 \\
10.00 & 14.00 \\
10.00 & 14.00 \\
10.00 & 14.00 \\
10.00 & 14.00 \\
10.00 & 14.00 \\
10.00 & 14.00 \\
\hline 10.00 & -14.00
\end{tabular}

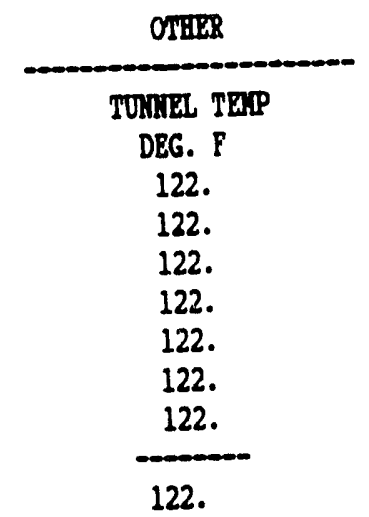

BLONER + 47m VOL, SCP 6776.1 BLOWIRR + 47w PLOW SCPH 225.84 P/A PCT, MEAS
BLOWER + 47m MASS,LB 509.2

BLOWBR + 47m FLON LB/KCIN 16.97

\section{FILTER DATA}

\begin{tabular}{|c|c|c|c|c|c|}
\hline & FILTER & SAYPLE VOL. & $\begin{array}{l}\text { AYG. SAMPLE SYS. } \\
\text { TETP DEG P }\end{array}$ & $\begin{array}{l}\text { GRIGIT GAIN } \\
\text { MTLLIGRAYS }\end{array}$ & $\begin{array}{l}\text { MG/SCP } \\
\text { OP SAMPLE }\end{array}$ \\
\hline SITION & NOUBER & SID.CO.FEE' & 68. & 3.295 & .152 \\
\hline 1 & P47-129\&13 & $\begin{array}{l}21.6 / 8 \\
21.701\end{array}$ & 69. & 4.027 & .186 \\
\hline
\end{tabular}

\section{PARTICULATE RESULTS}

AVG. SAIPLE ZONE TEYP, DEG F 122. DILUTION PACTOR 10.457

SPLIT EXHAOST VOL,SCF (SCA), LB/:MH $648.0(18.35), 1.62$

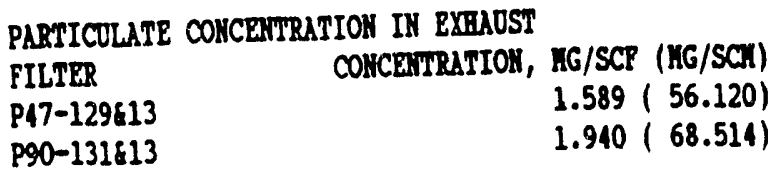

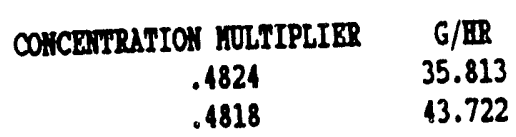

35.813

G/IP-BR .779

\begin{tabular}{|c|c|c|c|c|}
\hline & 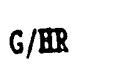 & G/EP-ER & collc. & \\
\hline & 51.213 & 1.113 & 130. PPHC & \\
\hline$\infty$ & 64.460 & 1.401 & 82. PPH & \\
\hline $\begin{array}{l}10 X \\
10 X, C O B R\end{array}$ & 527.061 & 11.458 & 412. PPII & \\
\hline $\begin{array}{l}\mathrm{NOX}, \mathrm{CORR} \\
\mathrm{CO2}\end{array}$ & 568.413 & $\begin{array}{l}12.357 \\
504 .\end{array}$ & & \\
\hline $\begin{array}{l}\mathrm{CO} 2 \\
02\end{array}$ & & 504. & $\begin{array}{r}2.44 \text { PCT } \\
17.19 \mathrm{PCT}\end{array}$ & \\
\hline NOX CORRI & NACTOR & 1.0785 & WET CORRECTIOH PACTOR & .9755 \\
\hline
\end{tabular}


GASEOOS and/Or PARTICOLATE EIISSIOHS

HOTCI 4

PROJECT 03-2681-809

TEST HO. RON 110.5

EHGIHE HODEL EID 2-567B

TEST CELLL LOCO LAB

BCR 1.758

BLONER COUITS 28131.

OBS. POWER, BIPP 78.0
TEST DATE 2/28/91

DIRSEL DP-2

TOIATR N0. 26

SAIPLE TIIR $20 \times 20 \quad .00$ KIH. BLONTR TERP. DEG. F 75. FOEL FLON,LB/KIN $\quad .54$
PROGRHA $=$ DD16B

BAROIEIER 29.08 III.BG.

HOIIDITY 85.57 GRAIIS B20/LB. AIR

LPE KO. 15 EXIG. LPE 10.0

SAPLE TIJE 47\% 18.72 III.

VRAS. EXB. FLOK,LB/KIH 33.87

AIR FLON, LB/RIIY
33.33

\begin{tabular}{|c|c|c|}
\hline \multicolumn{3}{|c|}{ LFE IKTORUATION } \\
\hline $\begin{array}{c}\text { IIHLT TEIP } \\
\text { DEG. F } \\
83 . \\
83 . \\
83 . \\
83 . \\
83 .\end{array}$ & $\begin{array}{c}\text { IXLET PRESS. } \\
\text { II. B20 } \\
.59 \\
.59 \\
.59 \\
.59 \\
.59\end{array}$ & $\begin{array}{c}\text { DIFF. PRESS. } \\
\text { IN. B20 } \\
3.920 \\
3.920 \\
3.920 \\
3.920 \\
3.930\end{array}$ \\
\hline IGE 83. & .59 & 3.922 \\
\hline
\end{tabular}

LPE VOL, SCP 3640.5

LFE PLON SCPI 194.52

F/A DRY, MEAS .0165
LFE MASS,LB. 273.5

LPE PLON LB/XIM 14.62

P/A CALC

.0149
BLONER IIPORNATION

\begin{tabular}{cc} 
IRLET PRESS. & DIPT. PRESS. \\
IH. B20 & IK. B20 \\
10.00 & 14.00 \\
10.00 & 14.00 \\
10.00 & 14.00 \\
10.00 & 14.00 \\
10.00 & 14.00 \\
\hline 10.00 & -14.00
\end{tabular}

OTGIRR

TOLATEL TEXP

DEG. $F$

122.

124.

124.

124.

124.

124.
BLOWIR + 47m VOL, SCF $1220.2 \quad$ BLOWZR + 47m USS,LB 317.1

BLOWTR + 47w FLOW SCFI 225.50 BLONER + 47m PLON LB/STH 16.94 F/A PCT, ITESS

$-9.8$

FILTER DRTA

$\begin{array}{clc} & \text { FILTER } & \text { SAIPLE VOL. } \\ \text { POSITION } & \text { NOUBER } & \text { SID. } \cong . \text { FEET } \\ 1 & \text { P47-125\&12 } & 13.673 \\ 2 & \text { P90-127\&12 } & 13.505\end{array}$

AVG. SAYPLE SYS. TR.P DEG F

69.

69.

WEIGHT GAIN
SILLIGRANS
2.529
3.295

HG/SCP

OF SANPLE

.185

.242

PARTICULATE RESULIS

$\begin{array}{lll}\text { AVG. SAYPLE ZONE TRIP, DEG P 124. } & \text { DILOTION FACTOR } & 7.280 \\ \text { SPLIT EXHAOST VOL,SCF (SCA), LB/KIM } & 579.7(16.42), & 2.33\end{array}$

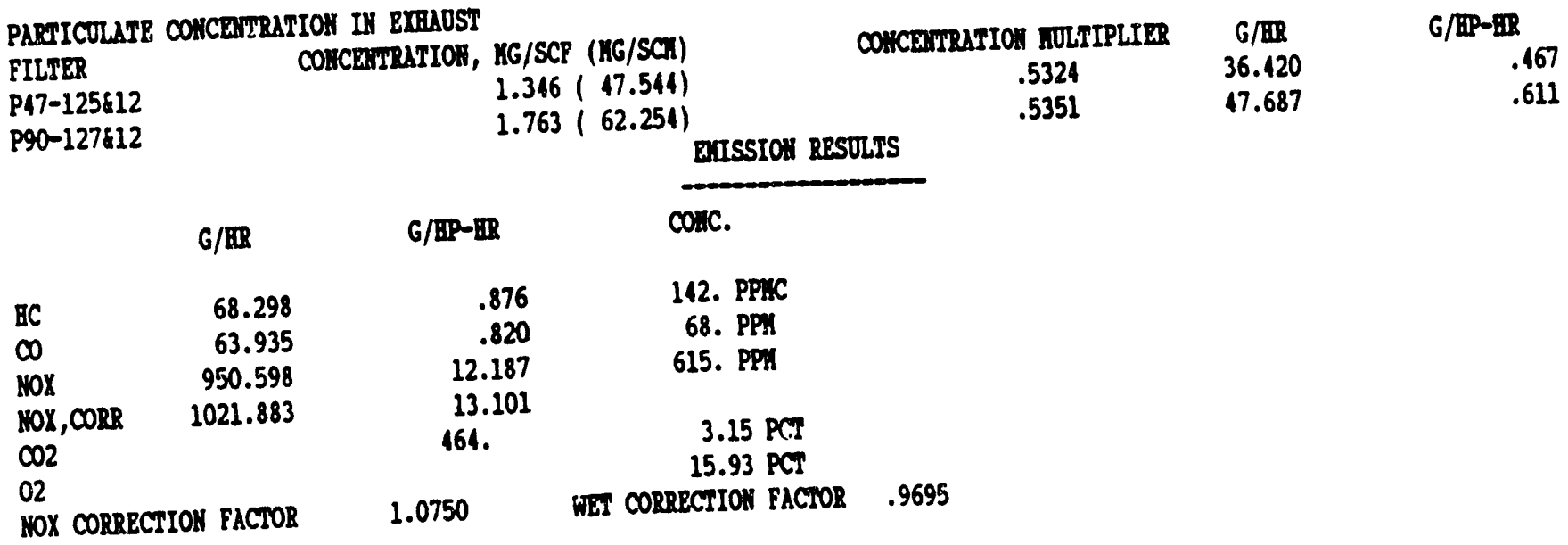


PROCRAL = EDI6B

TRST DATE 2/28/91

DIESEL DF-2

TUIARL HO. 26

SAIPLE TIKE $20 \times 20 \quad .00$ MIH.

BLONER TEIP. DEG. F 72.

FOEL FLOW, LB/HIM
BARONEIER 29.10 IH. BG.

HUIIDITY 80.42 GRAISS B20/LB. AIR

LFE 110. 15 EHG. LFE 110.0

SAYPLE TIIL $47 \mathrm{~m} 17.40$ IIH.

KERAS. EXH. FLON, LB/KIS 40.38

AIR PLOW,LB/KIN
39.62 OBS. POWER, BRP 114.0

LPE IIPORUATION

\begin{tabular}{cc}
\hline IILET TEYP & IRLET PRESS \\
DEG. F & IN. H20 \\
84. & .60 \\
84. & .60 \\
84. & .60 \\
84. & .60 \\
84. & .62 \\
\hline 84. & .60
\end{tabular}

BLOWER INFORATIOH

\begin{tabular}{l} 
IRLET PRESS \\
II. B20 \\
10.00 \\
10.00 \\
10.00 \\
10.00 \\
10.00 \\
\hline 10.00
\end{tabular}

OIHIBR

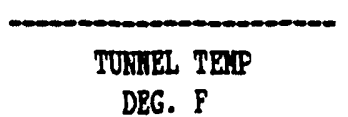

IN. 120

14.00

14.00

14.00

14.00

14.00

3.950

3.950

\subsection{0}

124.

124.

123.

123.

123.
LFE VOL, SCP 3398.7 LFE MLSS,LB. 255.4

LPE FLON SCPH 195.35 LPE FLON LB/HIN 14.68

P/A DRY, KEXS .0194
F/A CALC
BLONER + 47m VOL, SCF 3951.1 BLONER + 47m FLON SCTh 227.10 F/A PCT, IERS

$-2.5$
BLONER + 47W MLSS,LB 296.9

BLONRR + 47D FLOW LB/KIY 17.06

FILTER DATA

$\begin{array}{clc} & \text { FILTER } & \text { SAYPLE VOL. } \\ \text { POSITIOH } & \text { NIOIBER } & \text { STD.CO.FEET } \\ 1 & \text { P47-121612 } & 12.712 \\ 2 & \text { P90-123612 } & 12.693\end{array}$

\section{AVG. SAYPLE SYS. TEIP DEG I}

69.

69.

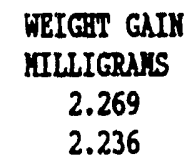

MG/SCP OP SAJPLE

.178

.176

PARTICULLTE RESULTS

AVG. SAYPLE 2ONE TEAP, DEG P 123. DILOTIOA PACTOR 7.153

SPLIT EXHADST VOL,SCP (SCI), LB/AII 552.4 ( 15.64)， 2.39

PARTICOLATE COHCEMTRATION II ETHAOST

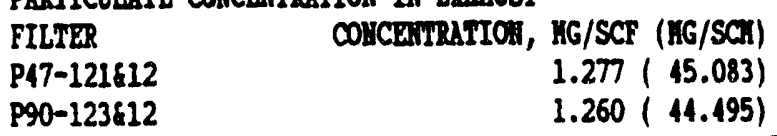

CO:CERTRLTO: MULTIPLIER

.5627

.5636
G/HR
41.166
40.629

G/HP-IR

.361

.356

\section{RISSIOA RESULTS}

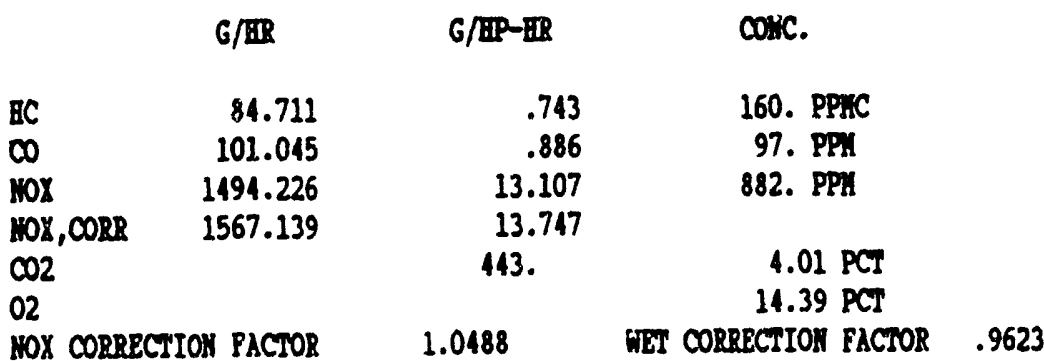


GASEOOS and/Or PARTICOLATE EIISSIOLS

HOTCH 6

PROJECT 03-2681-809

TEST 110. ROA HO. 3

EIGINE YODEL EID 2-567B

TRST CELLL LOCD LAB

BCR 1.758

BLONER COUTYS 45115.

OBS. POWER, BLP 148.0
TRST DATE 2/28/91

DIESEL DF-2

TOHIEL NO. 26

SAYPLE TIYIE $20 \times 20 \quad .00$ KIN.

BLONER TETP. DEG. F 71 .

FOEL FLON,LB/KIK

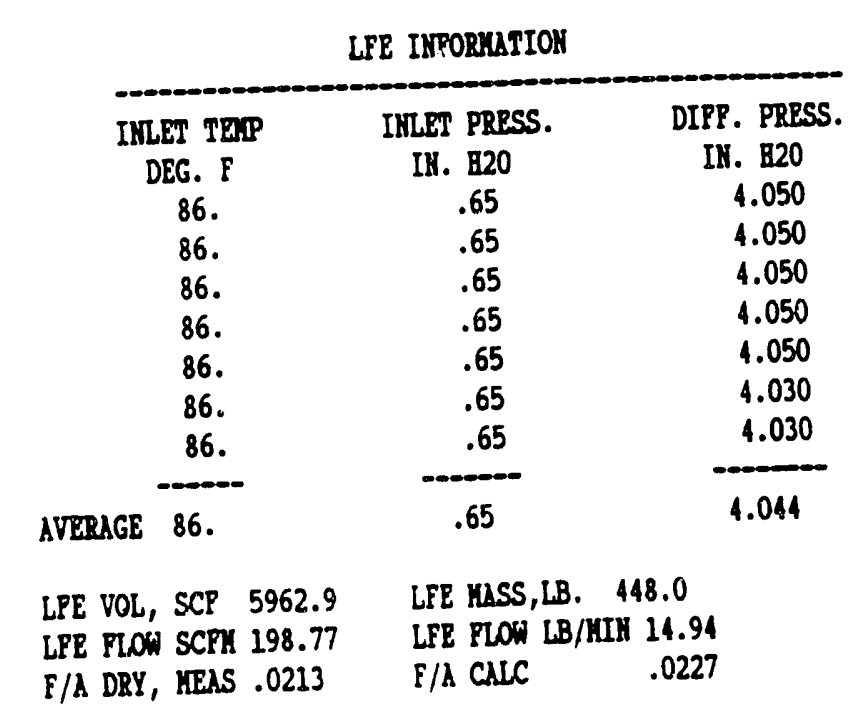

PROCRNH = EHD16B

BAROIEITER 29.14 IN. BG.

BUIIDITY 75.20 GRAIKS B20/LB. AIR

LPE 10. 15 ERT. LFE HO. 0

SAYPLE TIIIE 47m 30.00 IIIS.

YRAS. EXA. FLON,LB/KIII 47.63

AIR FLOA, LB/KIH

46.65
BLONER INFORYATION

\begin{tabular}{cc}
\hline IRLET PRESS. & DIFP. PRESS \\
II. H20 & IH. B20 \\
10.00 & 14.00 \\
10.00 & 14.00 \\
10.00 & 14.00 \\
10.00 & 14.00 \\
10.00 & 14.00 \\
10.00 & 14.00 \\
10.00 & 14.00 \\
\hline 10.00 & -14.00
\end{tabular}

TONAL TERP

DEG. $F$

121.

121.

121.

122.

122.

122.

121.

121.
BLONER + 47m VOL, SCP 6837.2 BLONER + 47m WASS,LB 513.7

BLONER + 47m FLON SCTI 227.92 BLONEB + 47W PLON LB/YIIH 17.13
F/A PCT, IRAS 6.4

\section{FILTER DATA}

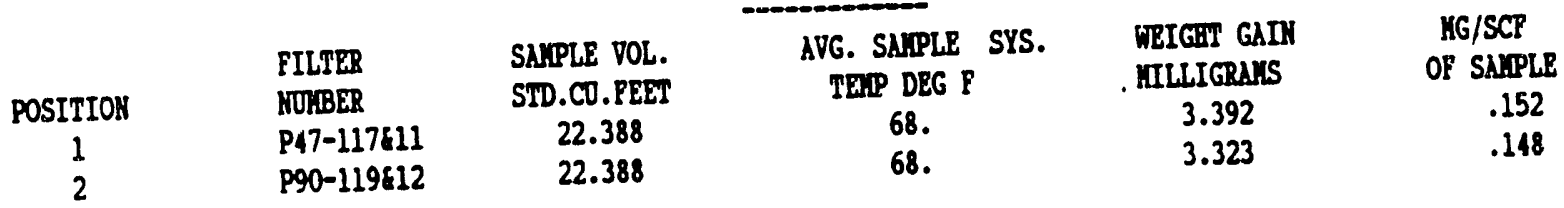

PARTICULATE RESULTS

AVG. SAYPLE ZONE TEIP, DEG F 121 . DILOTIOH PACTOR 7.820

SPLIT EXHADST VOL,SCF (SCR), LB/HIN $874.3(24.76) ， 2.19$

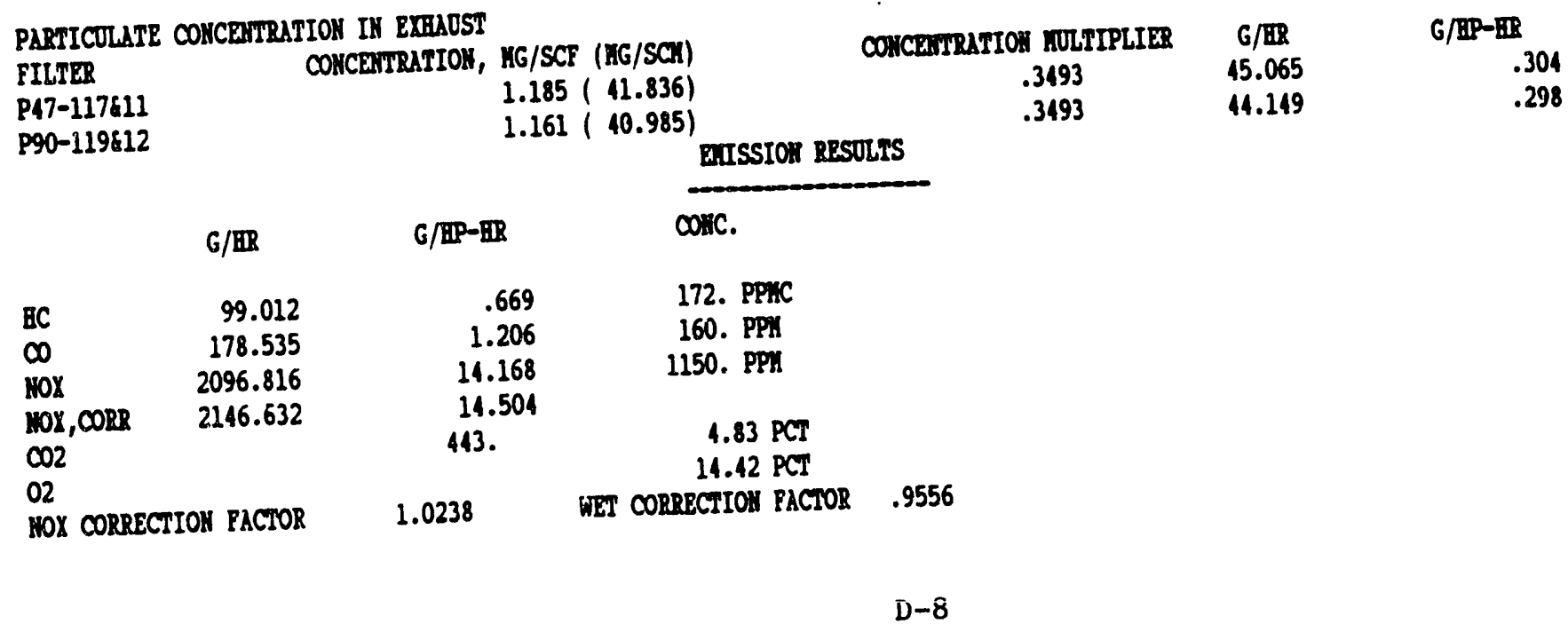


GASEOOS and/or PARICULATE EIISSIONS

NOTCB 7

PROJECT 03-2681-809

TRST DATB $2 / 28 / 91$

DIESEL DF-2

TOWHEL BO. 26

ETGIIT YODEL END 2-567B

ROH 110. 2

SATPLE TIIE $20 \times 20 \quad .00 \mathrm{MIK}$.

BCR 1.758

BLONER COUNTS 39775.

OBS. POWER, BHP 179.0

BLONER TEMP. DEG. F 71.

FOEL FLON, LB/KIIN

1.22

PROGRAY = ED16B

BARONETER 29.18 II. BG.

BUUIIDITY 59.78 GRAILS B20/LB. AIR

LPE W0. 15 EIG. LFE HO. 0

SAYPLE TIHE 47m 26.44 IIIN.

YRAS. EXB. PLOW,LB/YIN 54.42

AIR FLON, LB/MIH

53.20

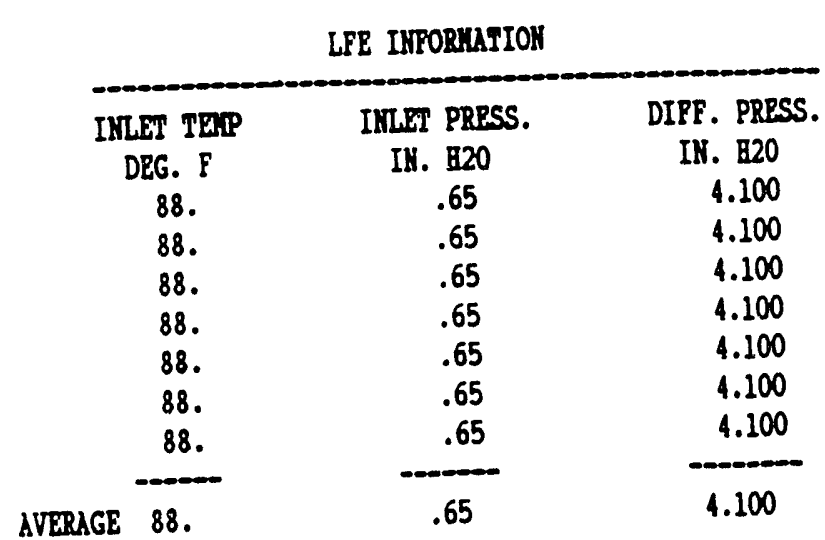

\section{BLOWER INPORYLATION}

IRLET PRESS. DIPP. PRESS.

IN. 120

10.00

10.00

10.00

10.00

10.00

10.00

10.00

10.00

BLONER + 47m VOL, SCP 6035.7

BLONER + 47 II FLON SCTH 228.31

E/A PCT, IEAS

10.1

PILTER DATA

$\begin{array}{ccc}\text { AVG. SAYPLE SYS. } & \text { WEIGRT GAIN } & \text { MG/SCP } \\ \text { TEIP DEG F } & \text { MILLIGRAHS } & \text { OF SAPLE } \\ 71 . & 3.121 & .163 \\ 71 . & 3.252 & .169\end{array}$

PAPTICULATE RESOLTS

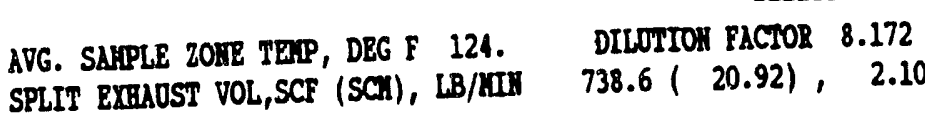

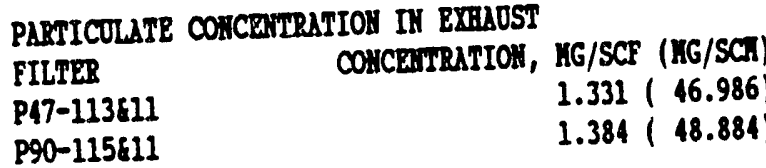

\begin{tabular}{lr} 
& \multicolumn{1}{c}{ G/HR } \\
BC & 150.811 \\
$C$ & 316.210 \\
YOX & 2456.928 \\
$110 X, C O R R$ & 2405.404 \\
$C O 2$ & \\
O2 & \\
MOX CORPECTIOH FACTOR
\end{tabular}

G/AP-BR

.843

1.767

13.726

13.138

453.

$5.43 \mathrm{PCT}$
$13.16 \mathrm{PCT}$

.9790 GET CORRECTIOH FACIOR .9514

\section{BIISSIOH RESULTS}

coisc.

237. PPHC

257. PPI

1223. PPI
CONCEATRATIOH IULTIPLIER G/HR G/HP-ER

$.4264 \quad 57.825$

.4257

60.161

.323
.336 
GASEOOS and/Or PARTICOLATE EMISSIONS

HOTCH 8

PROJECT 03-2681-809

TEST $10 . \quad$ RON 10.1 ENGINE HODEL END 2-567B

TEST CELL LOCO LAB

BCR 1.758

BLONER COCNTS 30246.

OBS. PONER,BHP 206.0
TEST DATE $2 / 28 / 91$

DIESEL DF -2

TOMARL NO. 26

SAIPLE TIKE $20 \times 20 \quad .00$ KIH.

BLOKIER TEIP. DEG. F 70. FOEL FLON, LB/KIH $\quad 1.47$
PROGRAY $=$ EID16B

BAROILTER 29.00 IN. BG.

BOSTDITY 68.95 GRAIRS H20/LB. AIR

LFE 10. 15 EIG. LFE 10.0

SAMPLE TIME 47m $20.10 \mathrm{KIH}$.

MEAS. EXH. FLON,LB/KIH 60.20

AIR FLON,LB/HIM $\quad 58.73$

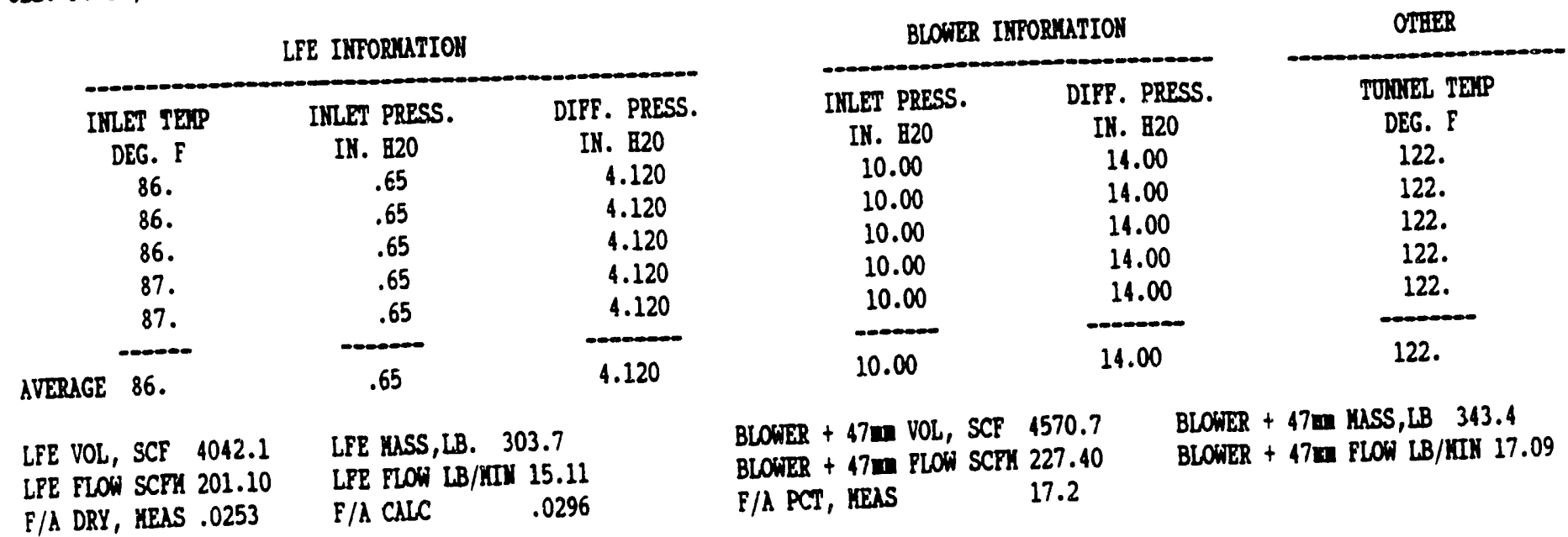

FILTER DATA

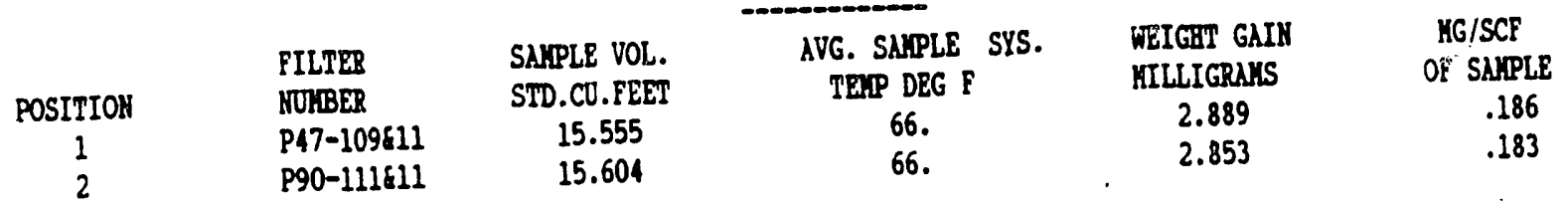

PARTICULATE RPSULIS

AVG. SAYPLE 2ONE TEAP, DEG F 122 . DILOTIOH FACTOR 8.647

SPLIT EXBAOST VOL,SCP (SCA), LB/KIH 528.6 ( 14.97)， 1.98

\begin{tabular}{|c|c|c|c|c|}
\hline \multicolumn{5}{|c|}{ PARTICULATE CONCERTRATIOA IH EXHAOST } \\
\hline FILTER & COHCEITRATIOH, MG/SCF (HG/SCA) & .5559 & 77.199 & .375 \\
\hline $\begin{array}{l}P 47-109 \$ 11 \\
P 90-111811\end{array}$ & $\begin{array}{l}1.606(56.708) \\
1.581(55.826)\end{array}$ & .5512 & 76.000 & .369 \\
\hline & & OLTS & & \\
\hline
\end{tabular}

$1.606(56.708)$

$1.581(55.826)$

EILSSIOH RESULTS

COIIC.

G/EP-BR

$\begin{array}{ll}\mathrm{BC} & 140.373 \\ C & 668.319\end{array}$

$\begin{array}{ll}\mathrm{BC} & 140.373 \\ C & 668.319\end{array}$

HOX 2892.032

HOX, CORR 2879.349

$\mathrm{CO} 2$

02

NOX CORRECTION FACTOR

$\begin{array}{rr}.681 & \text { 212. PPHC } \\ 3.244 & 526 . \text { PPI } \\ 14.039 & \text { 1395. PPH } \\ 13.977 & \\ 473 . & 6.32 \mathrm{PCT} \\ & 11.90 \mathrm{PCT}\end{array}$

.9956
WET CORRECTION FACTOR .9435

$$
\text { D-10 }
$$


APPENDIX E

E-1 

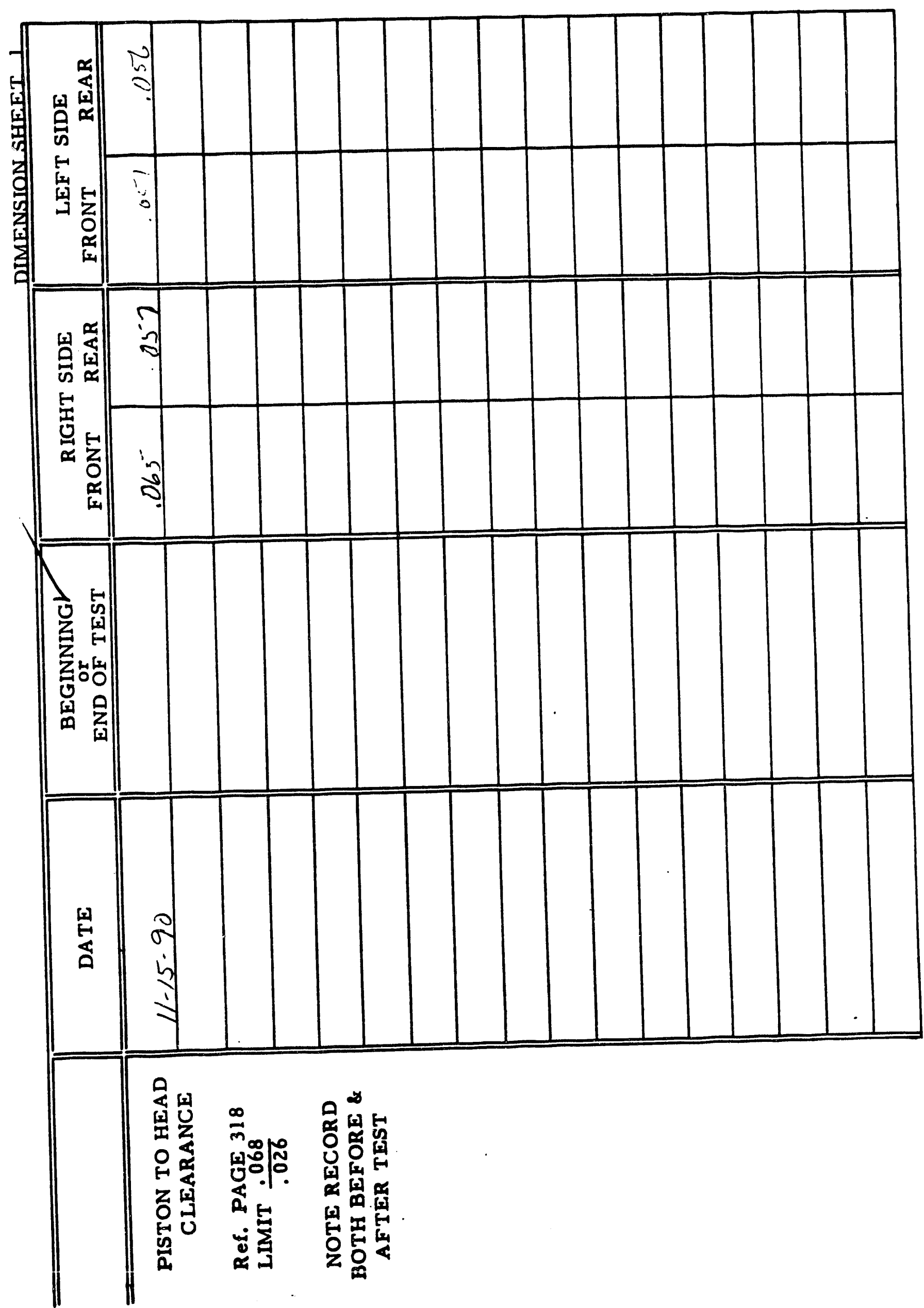


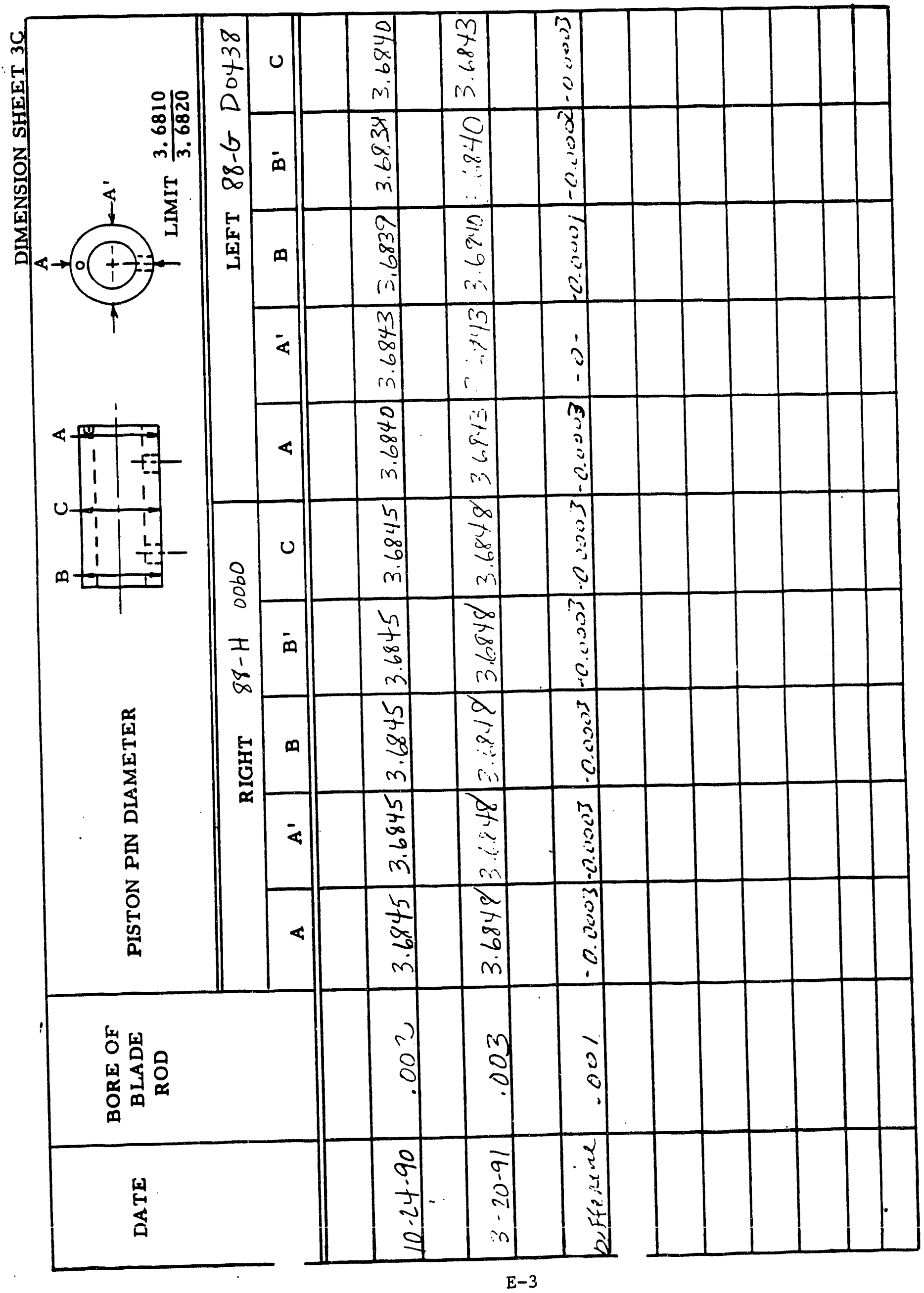




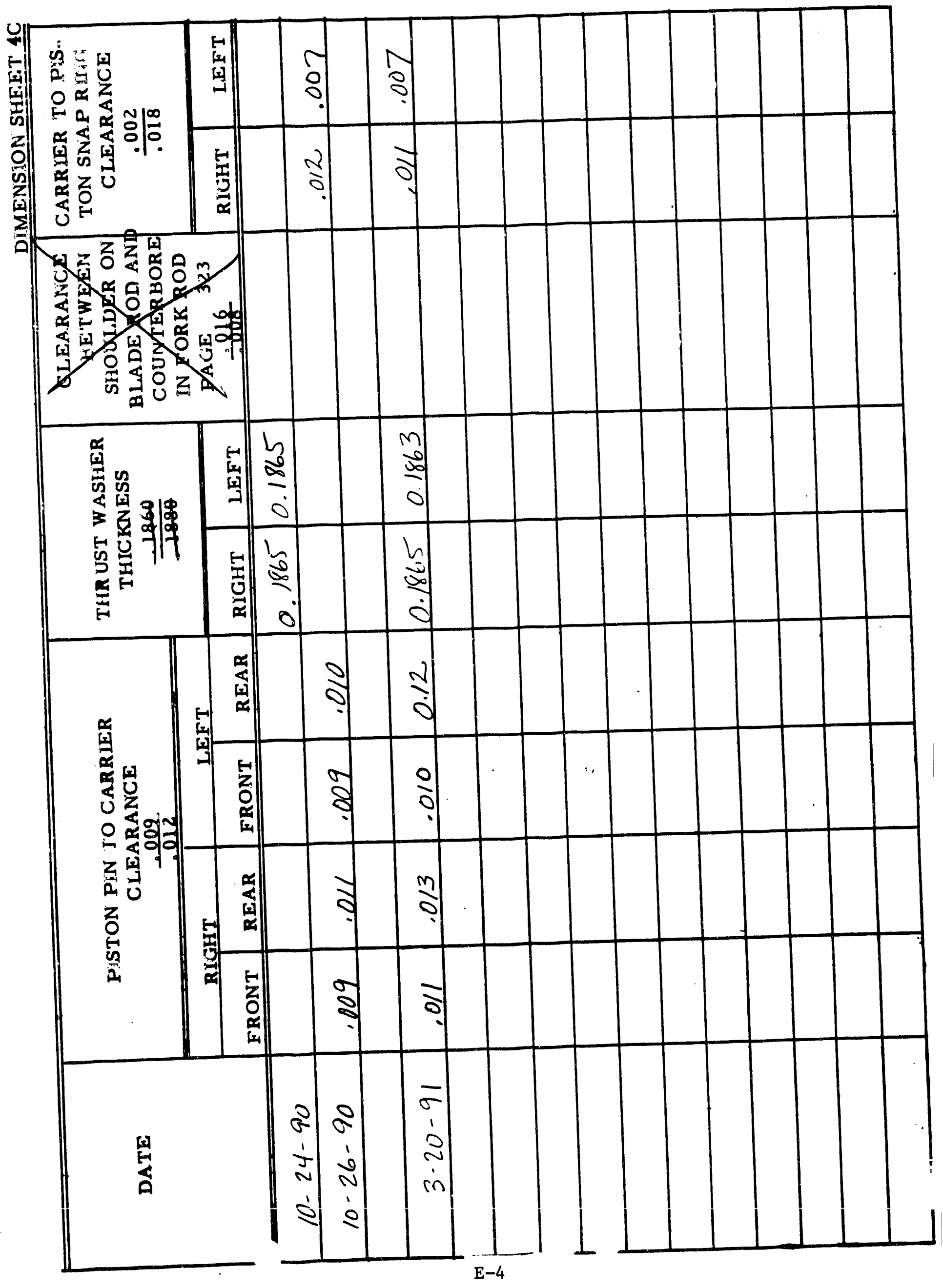




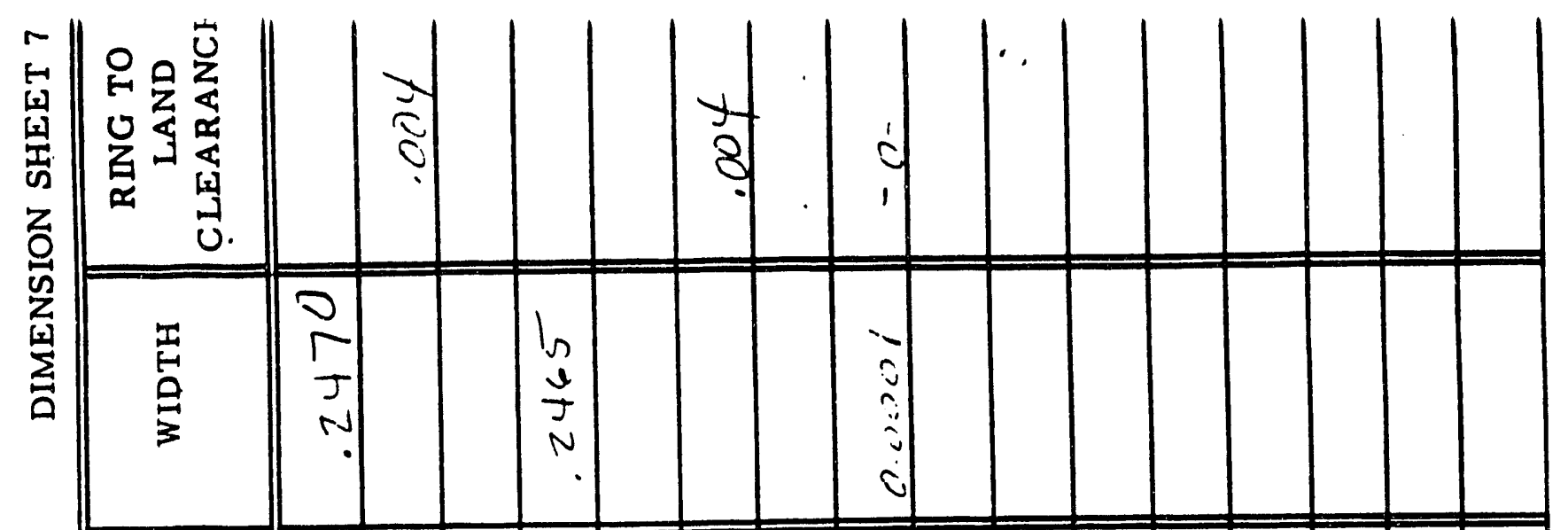

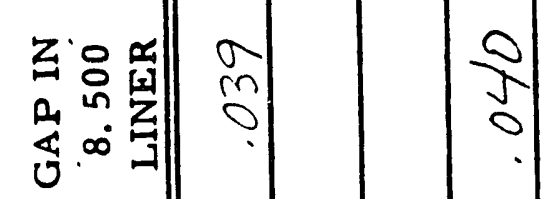

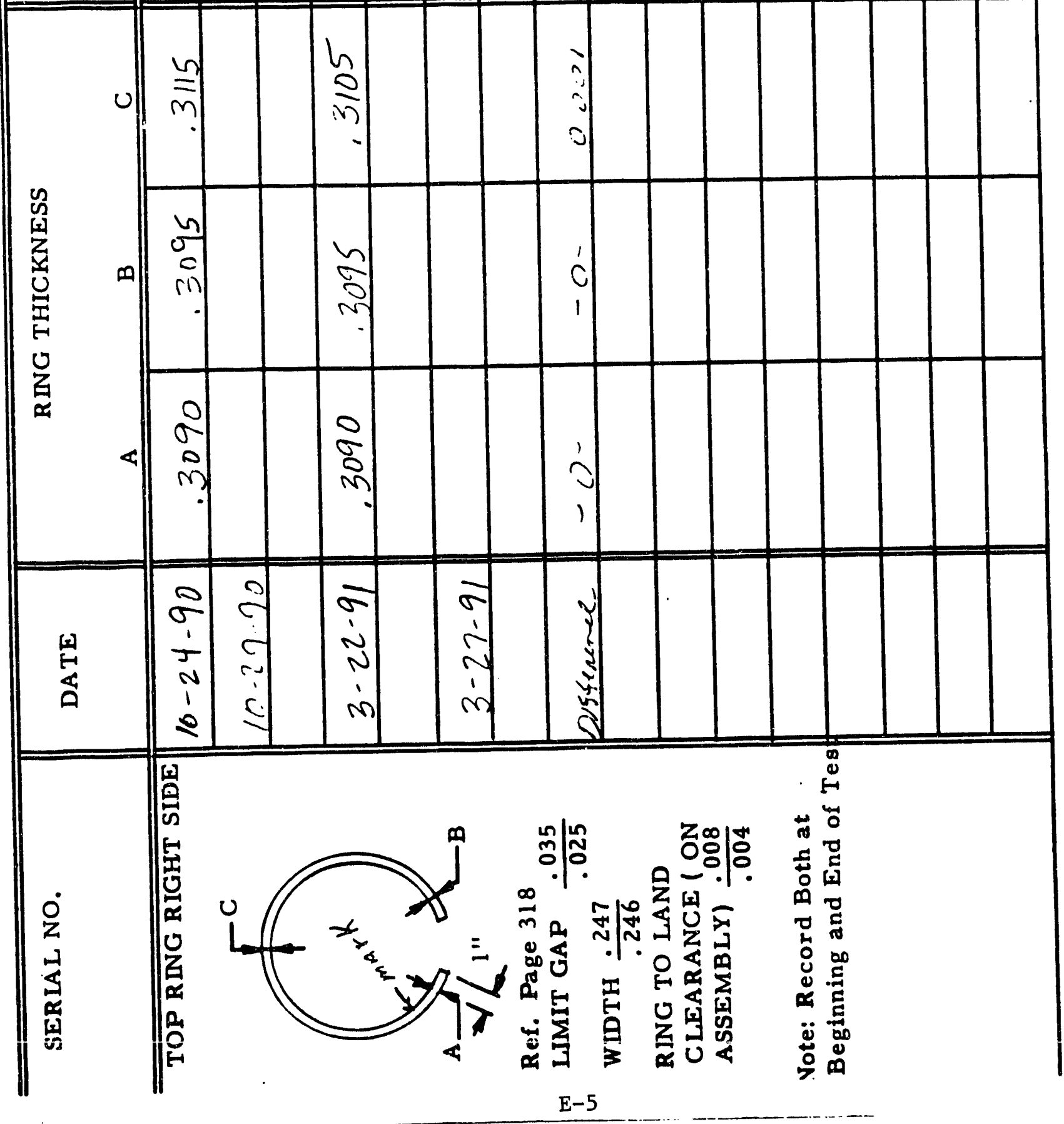



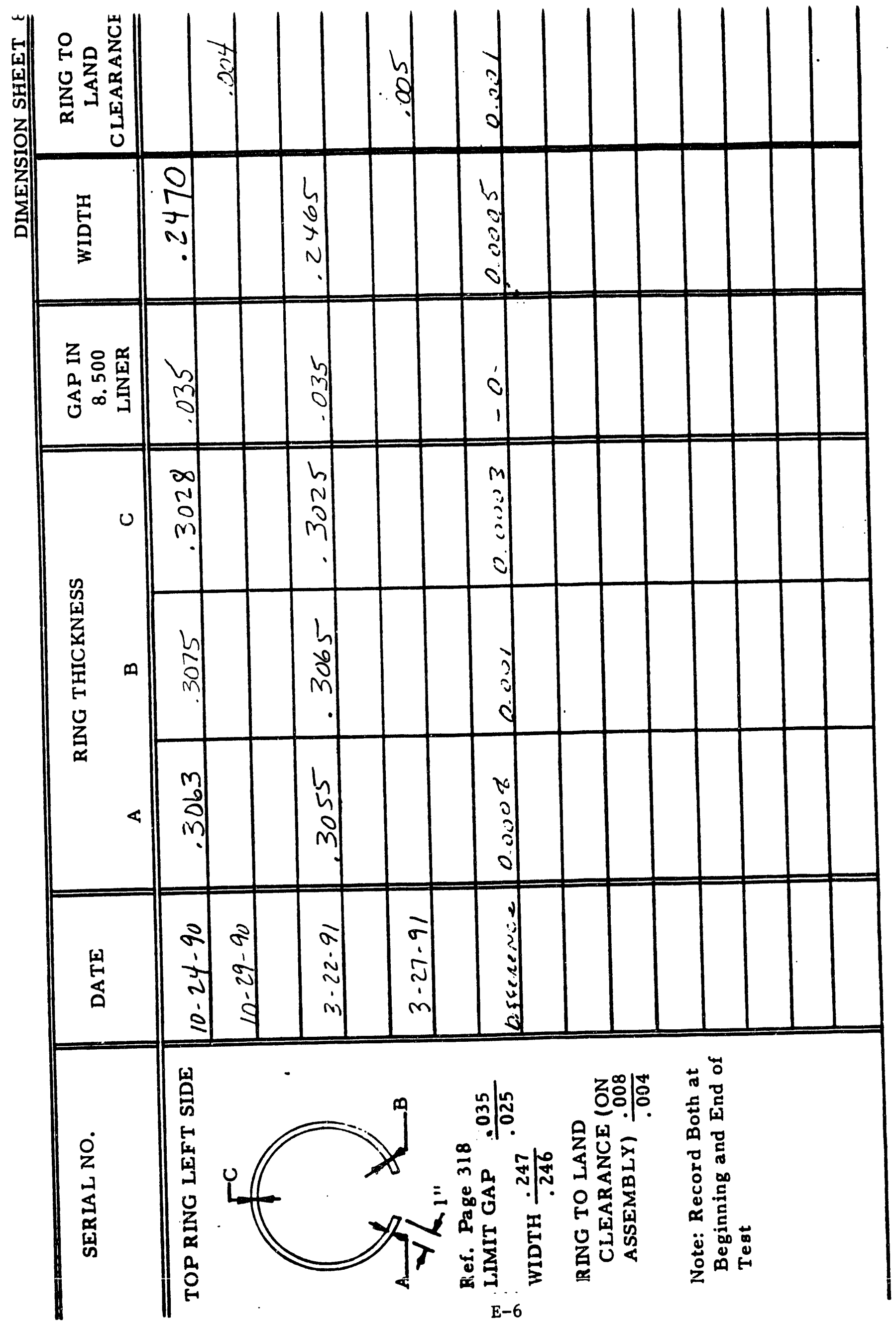
에

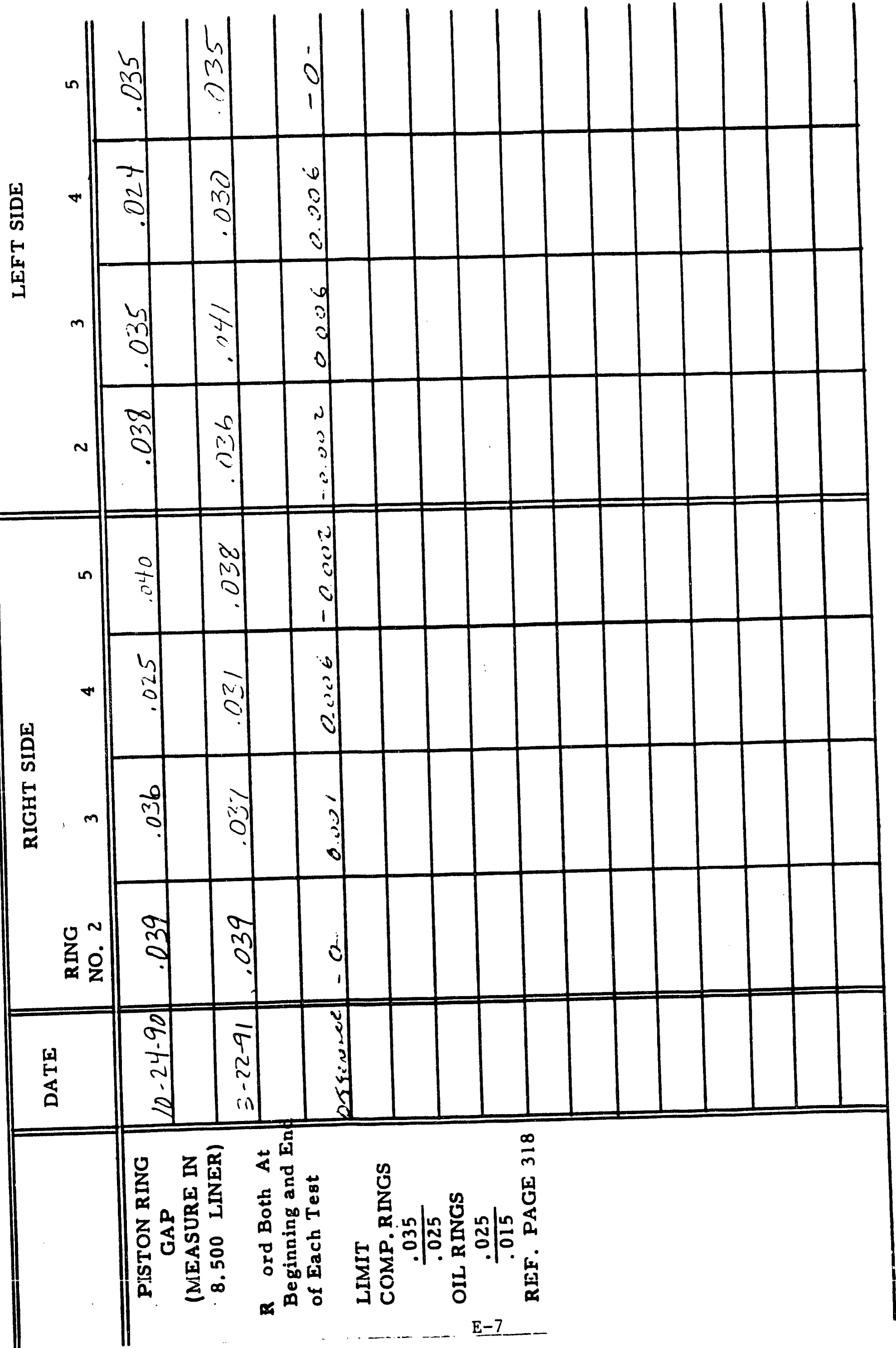




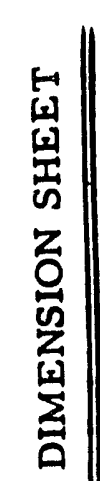
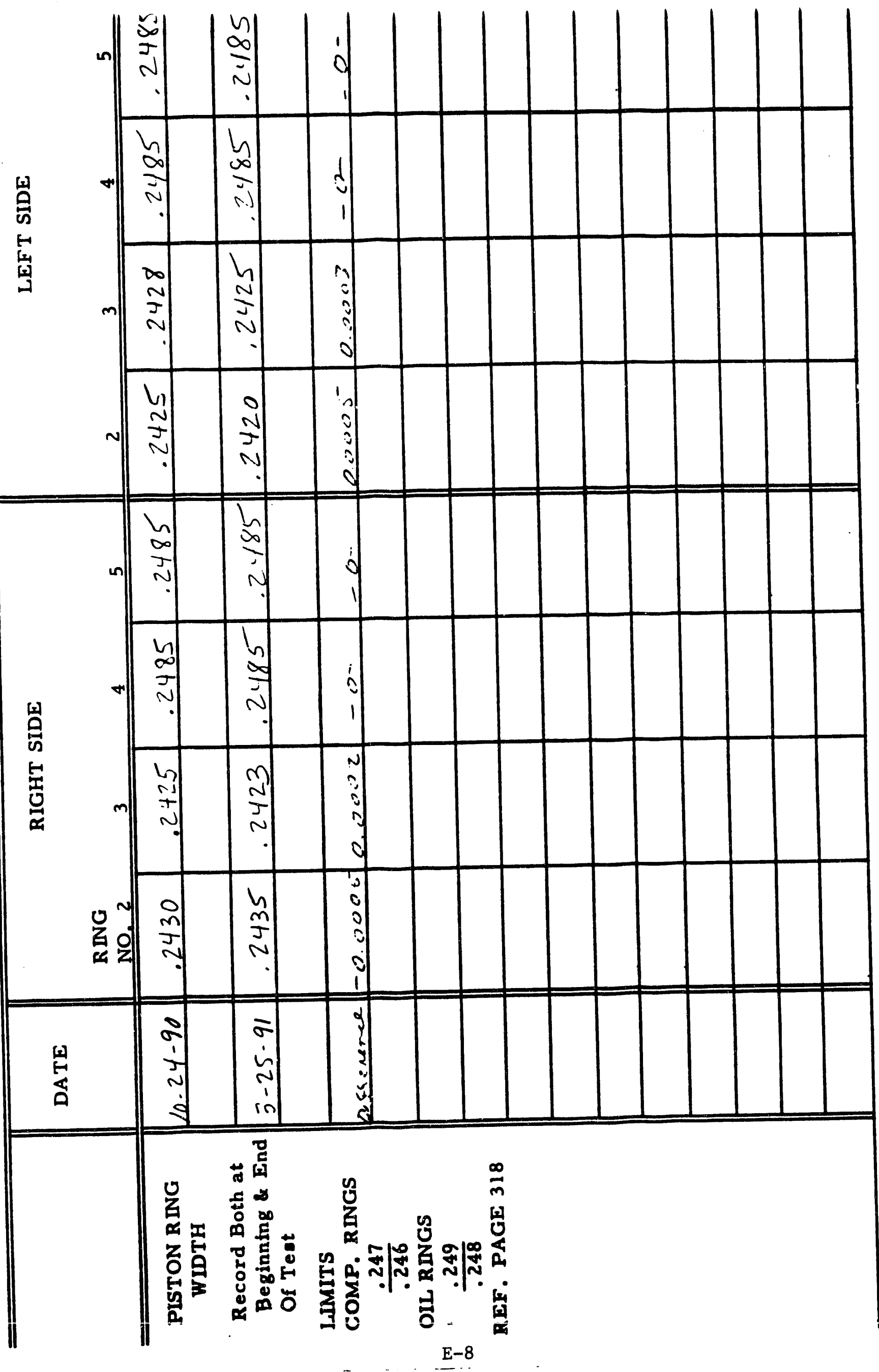

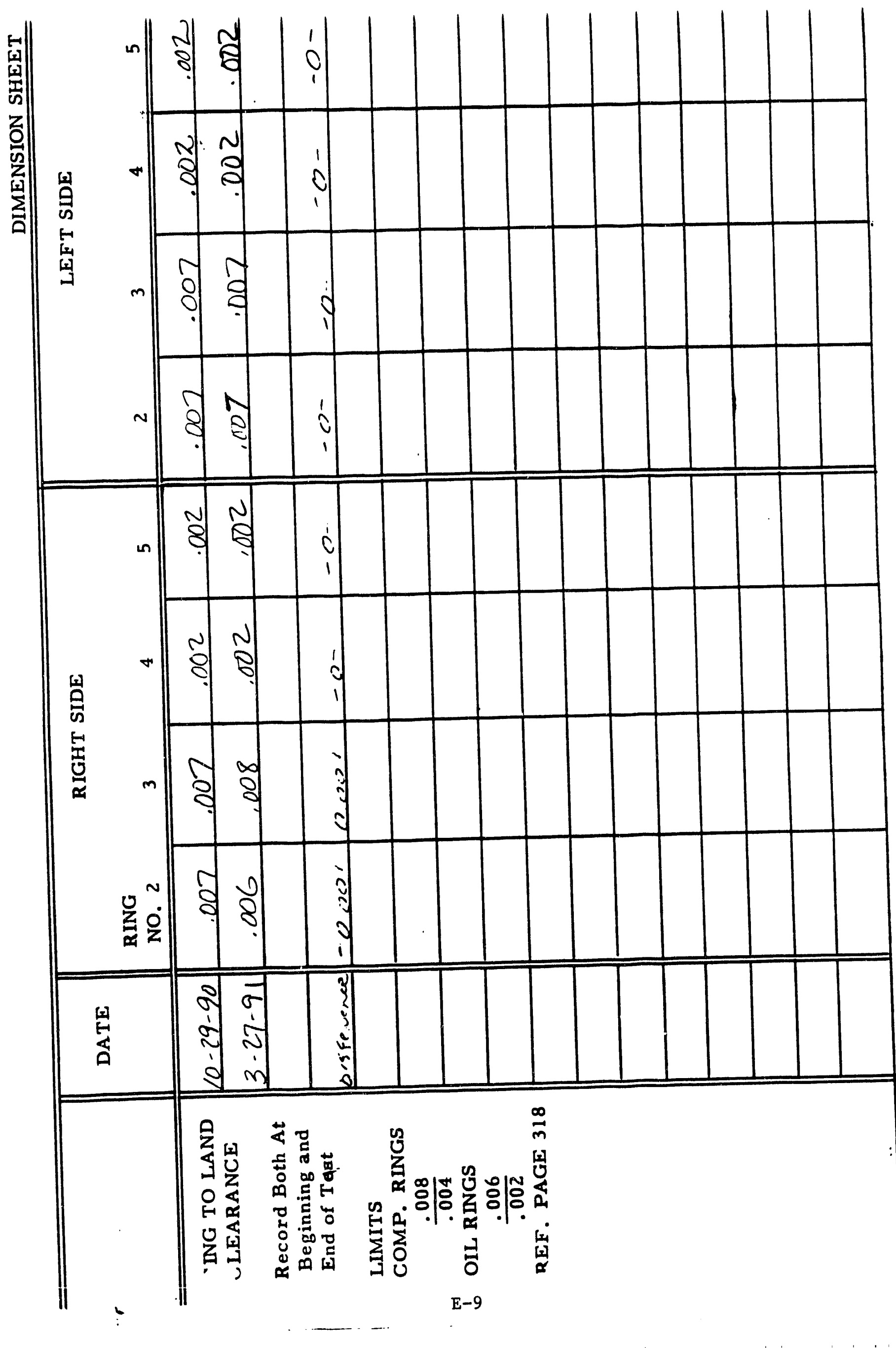


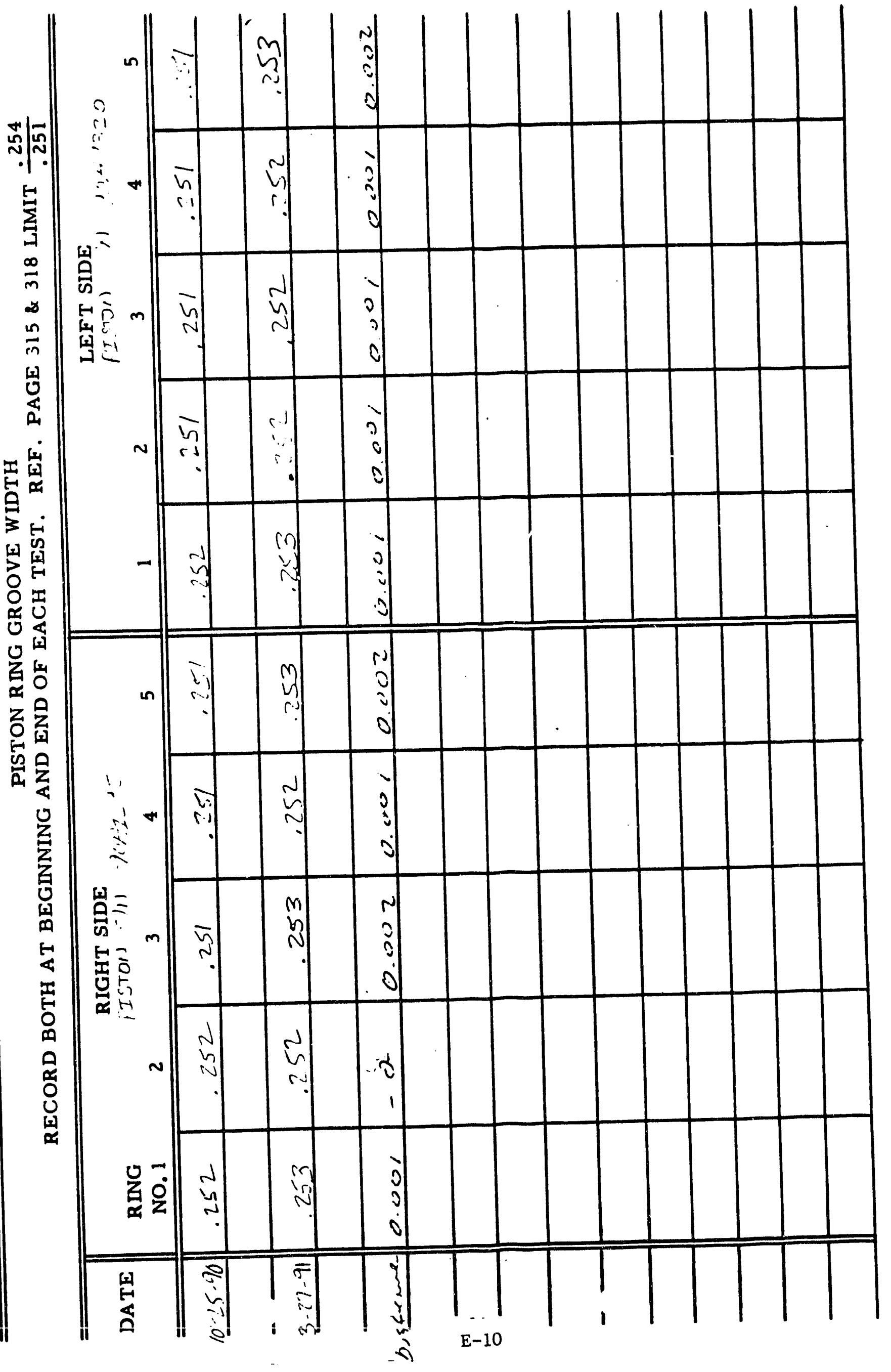




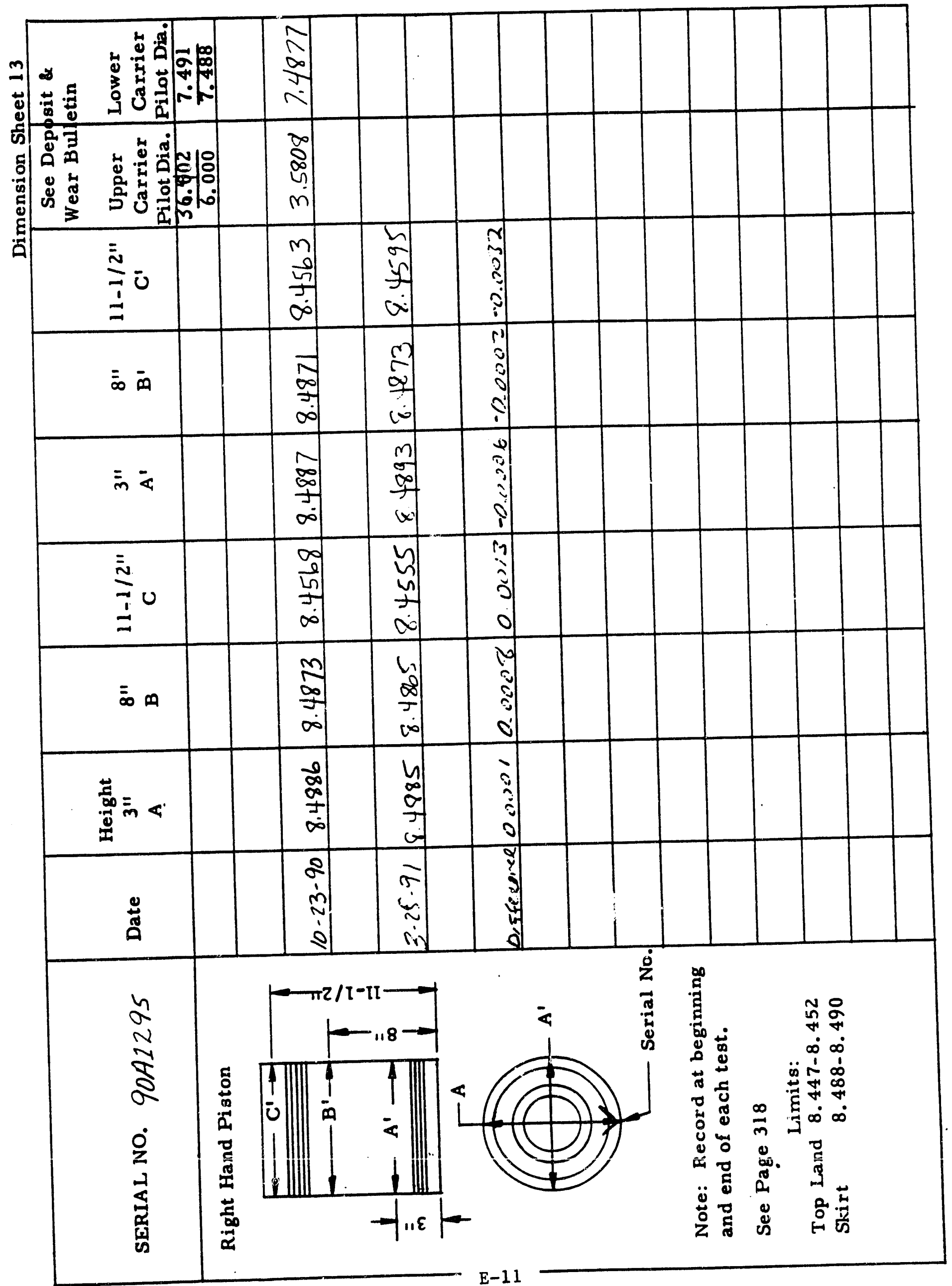




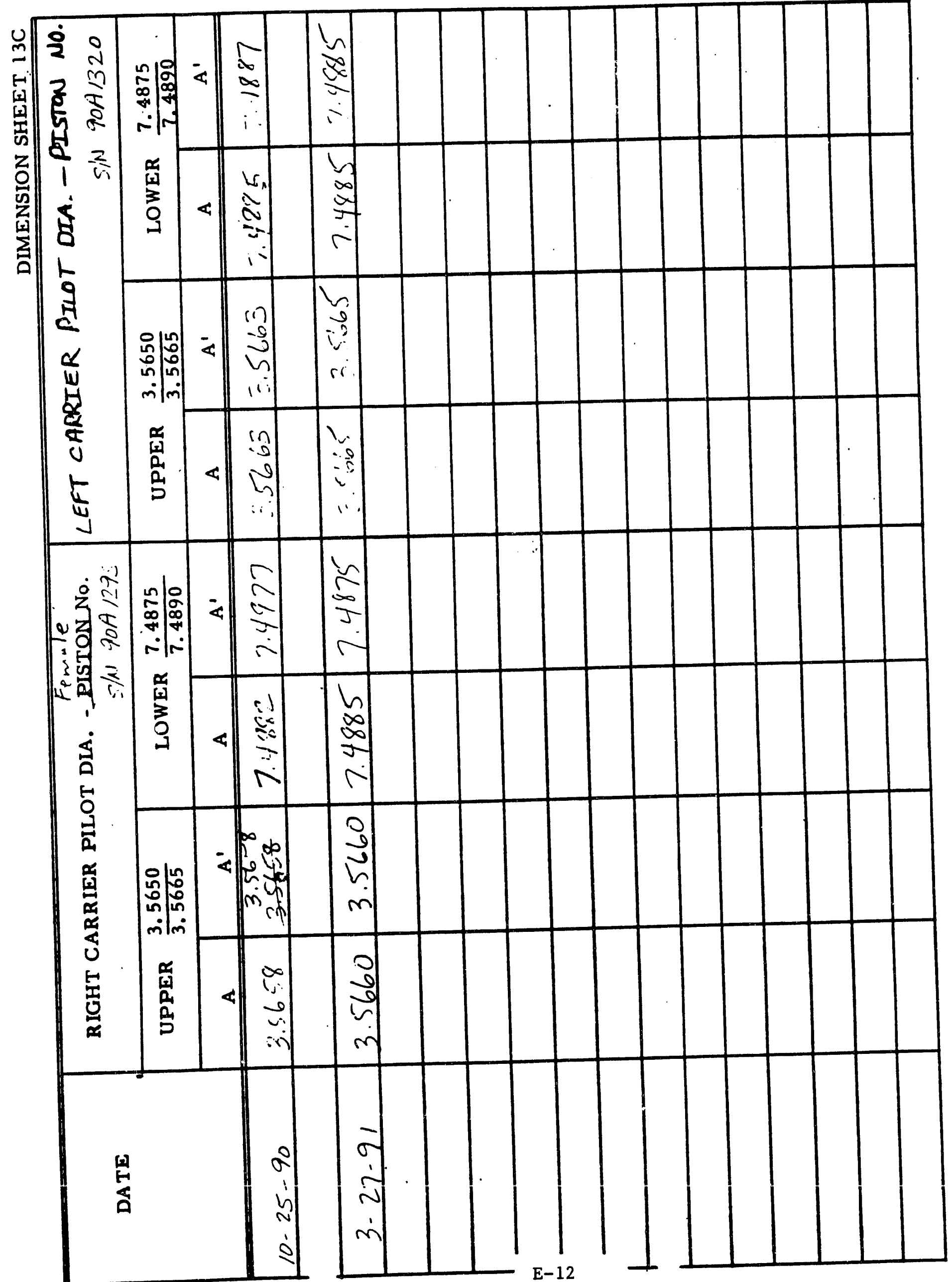




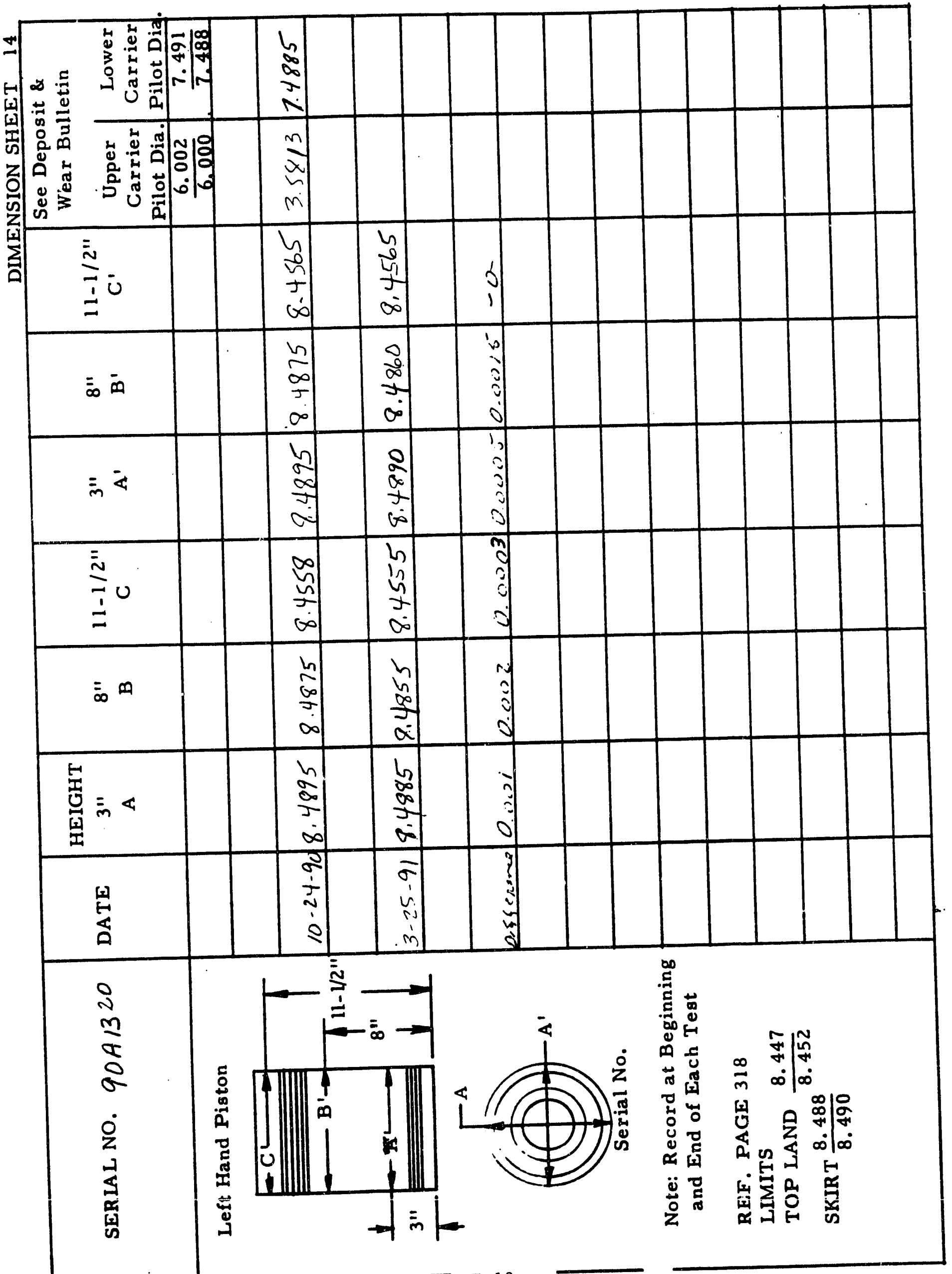



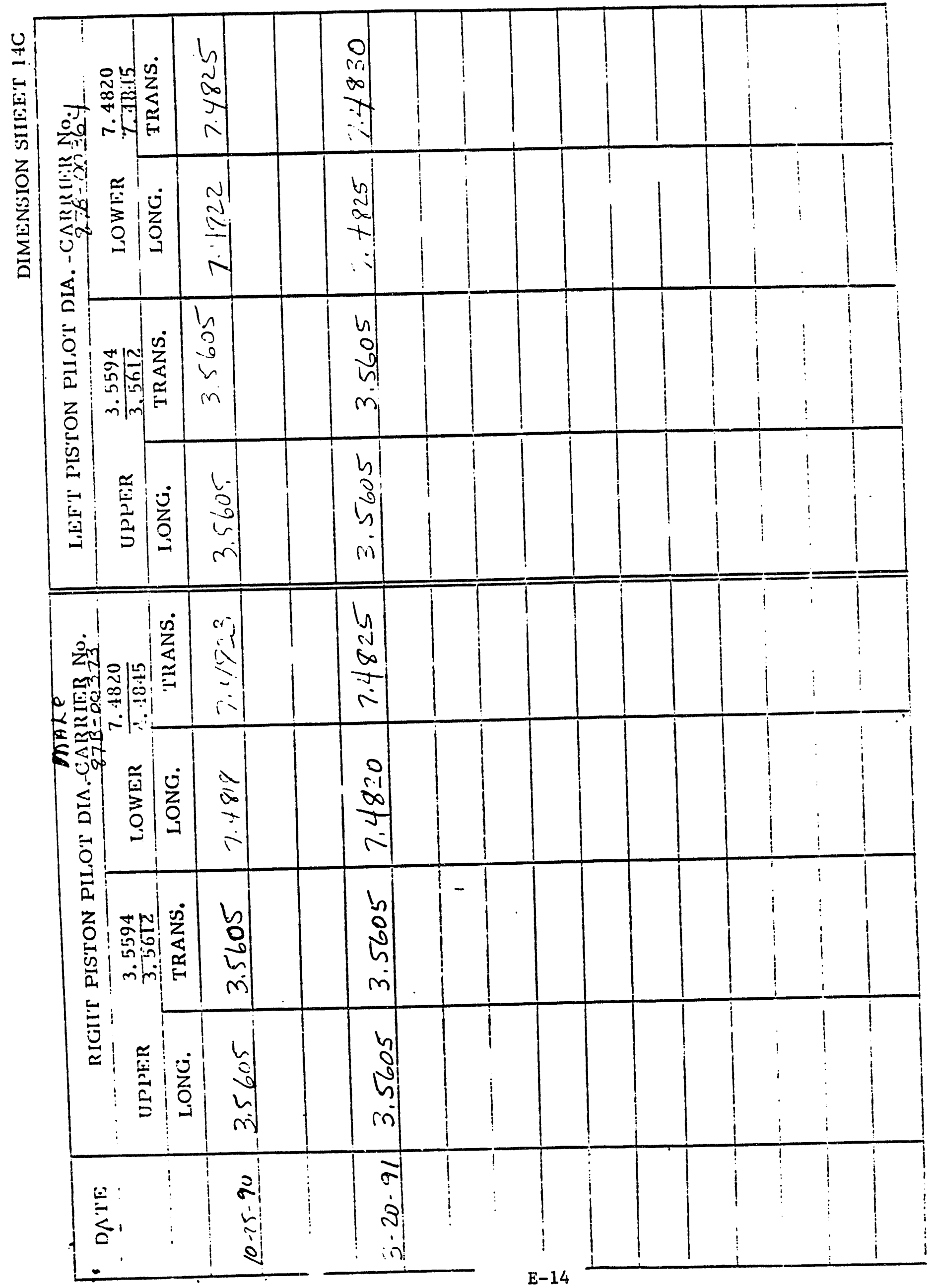


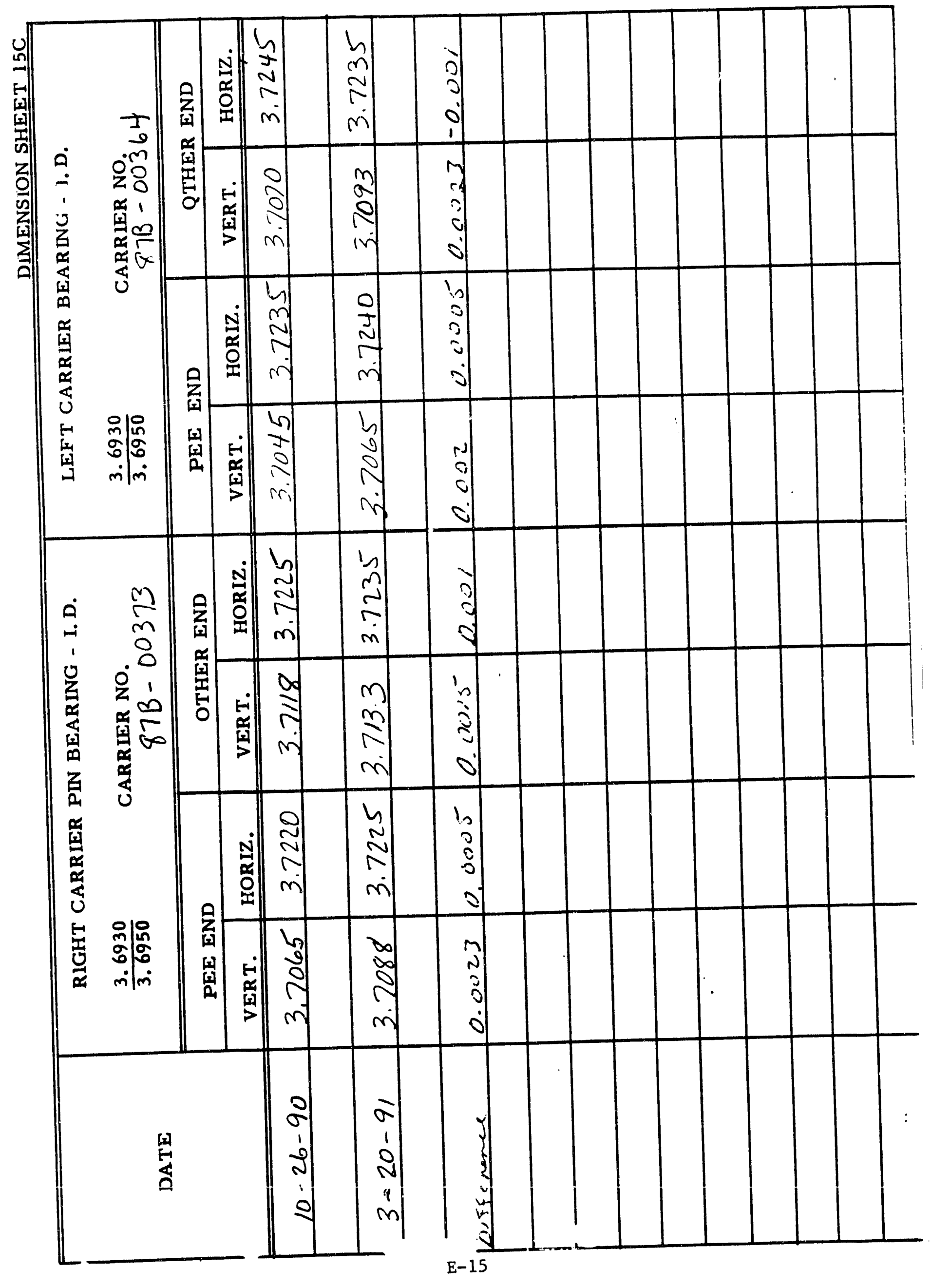




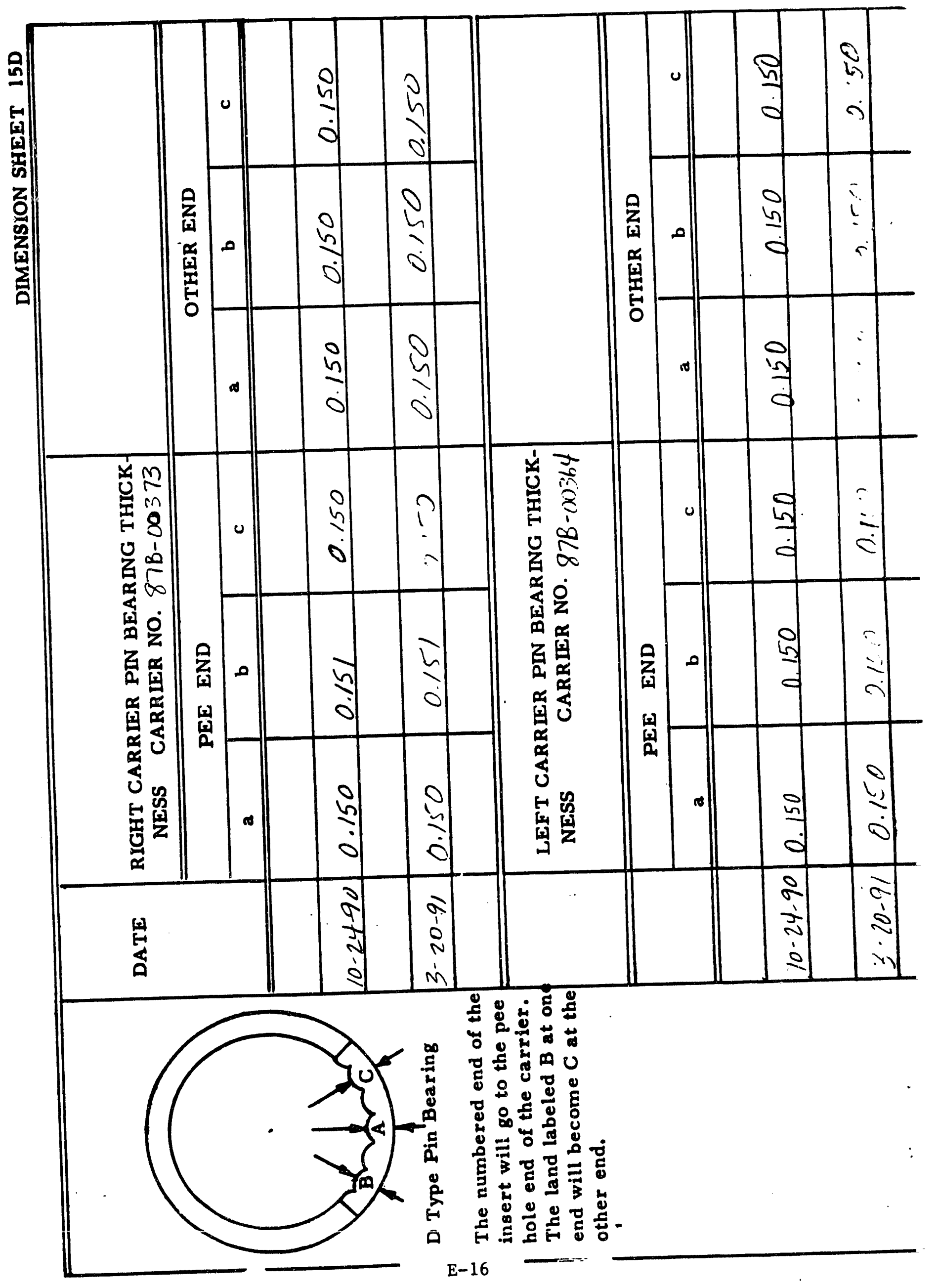



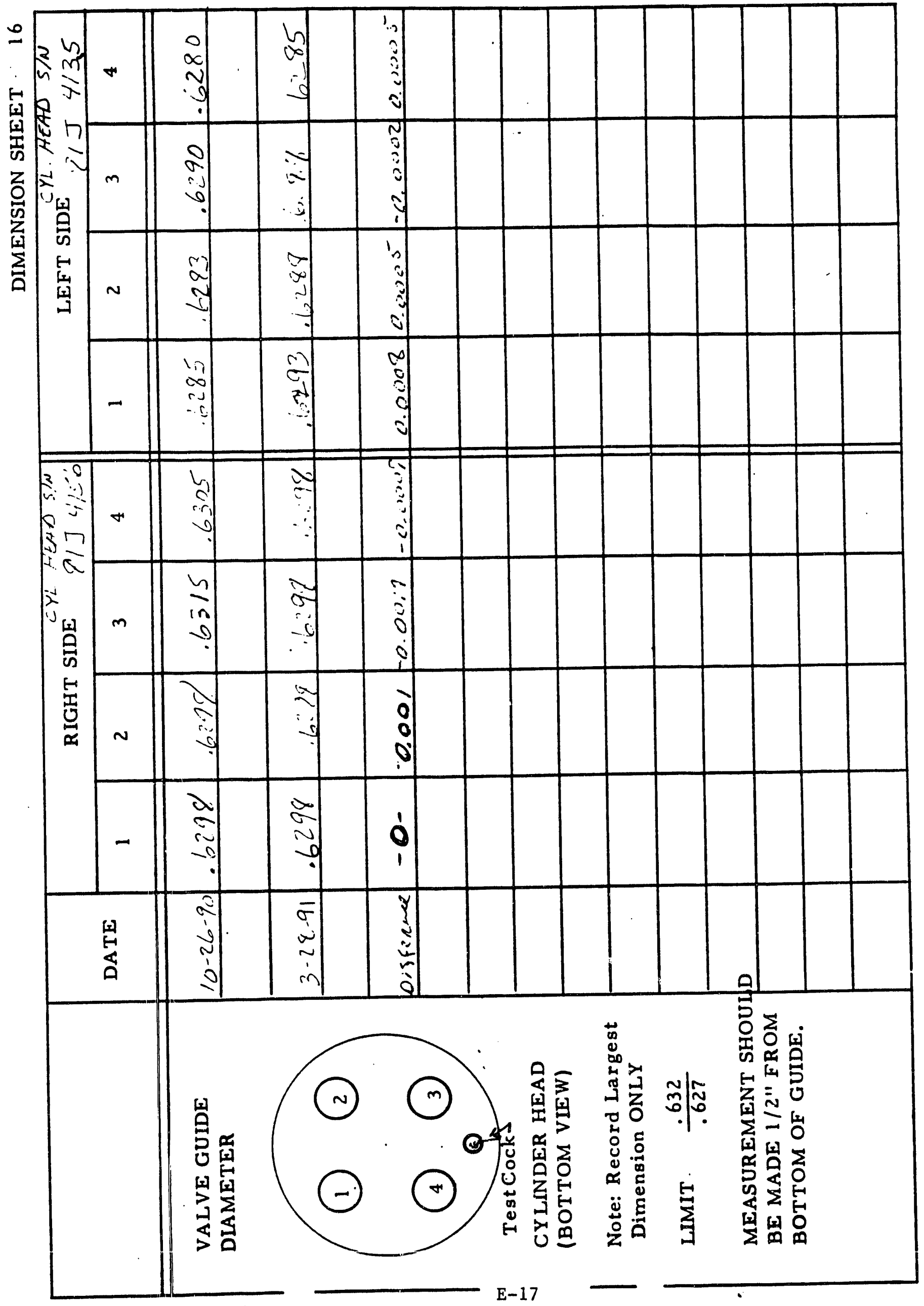


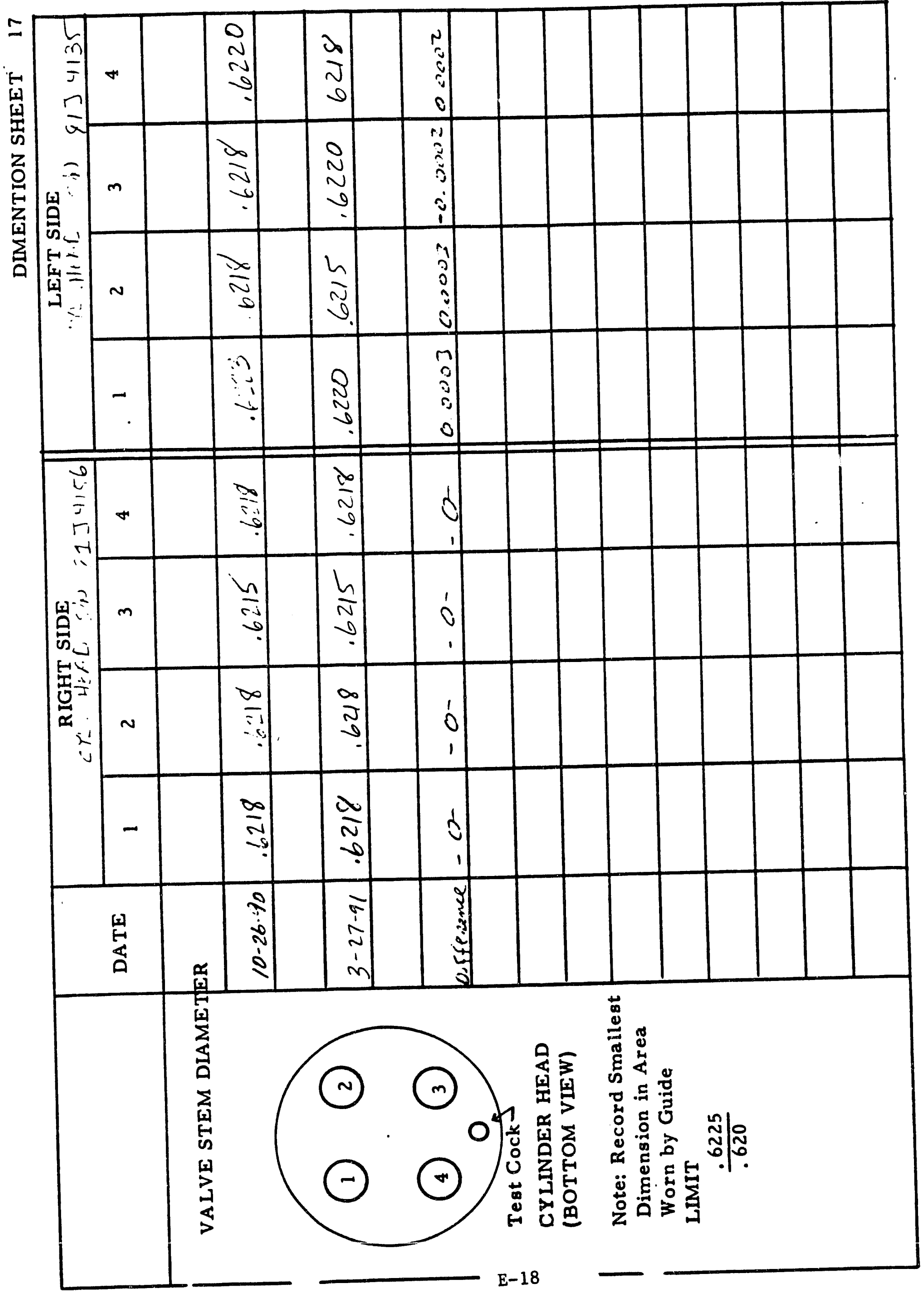



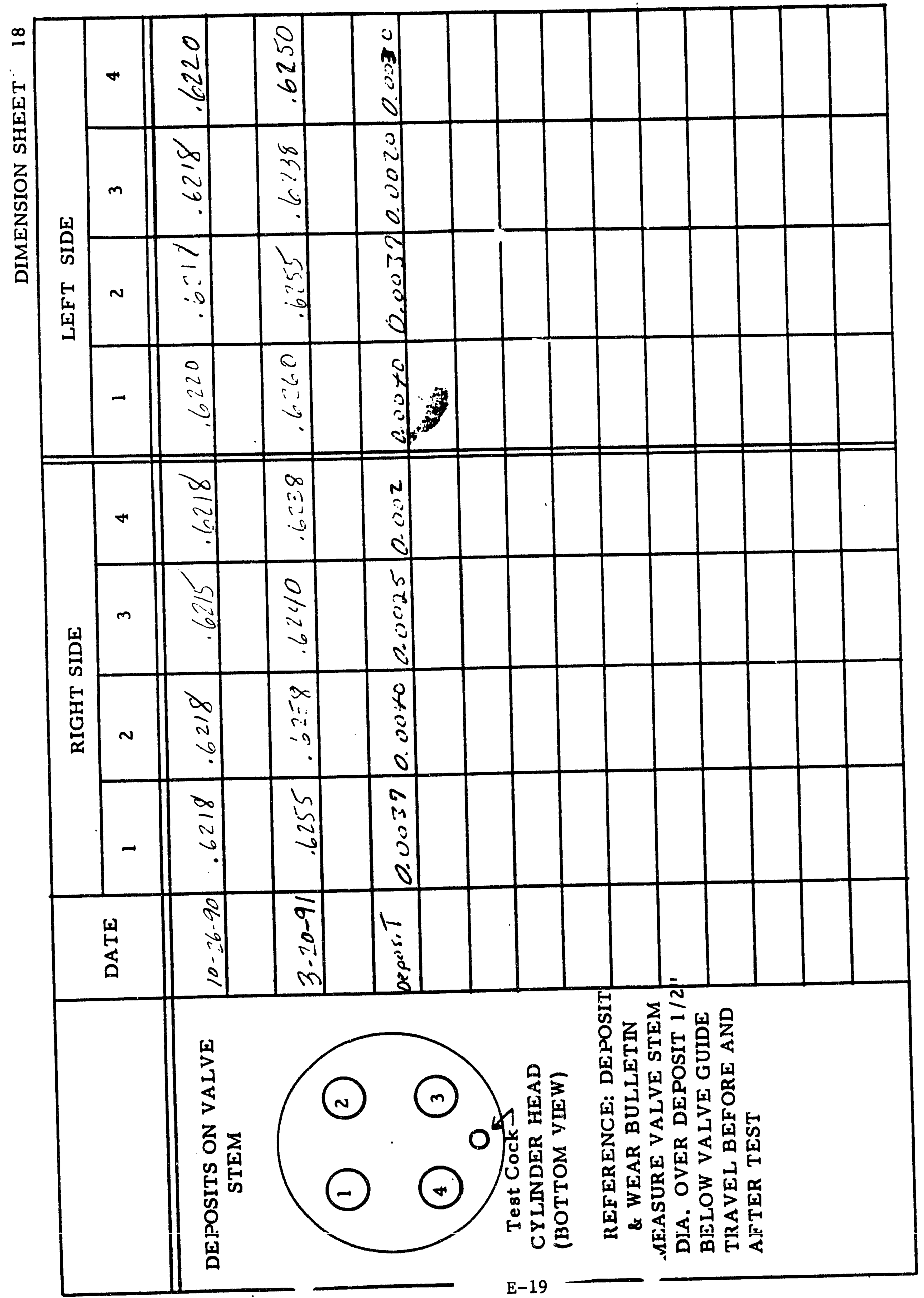

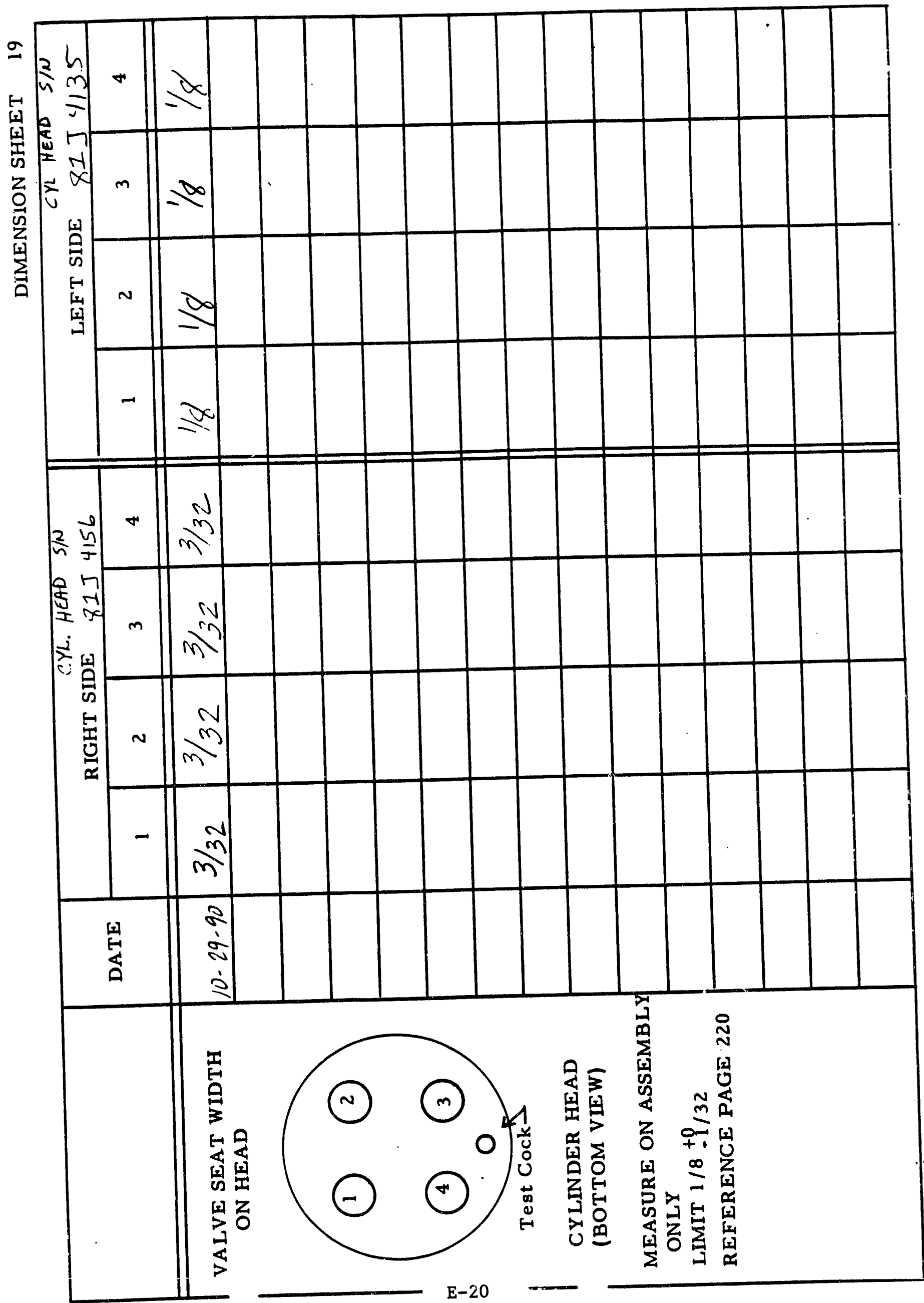


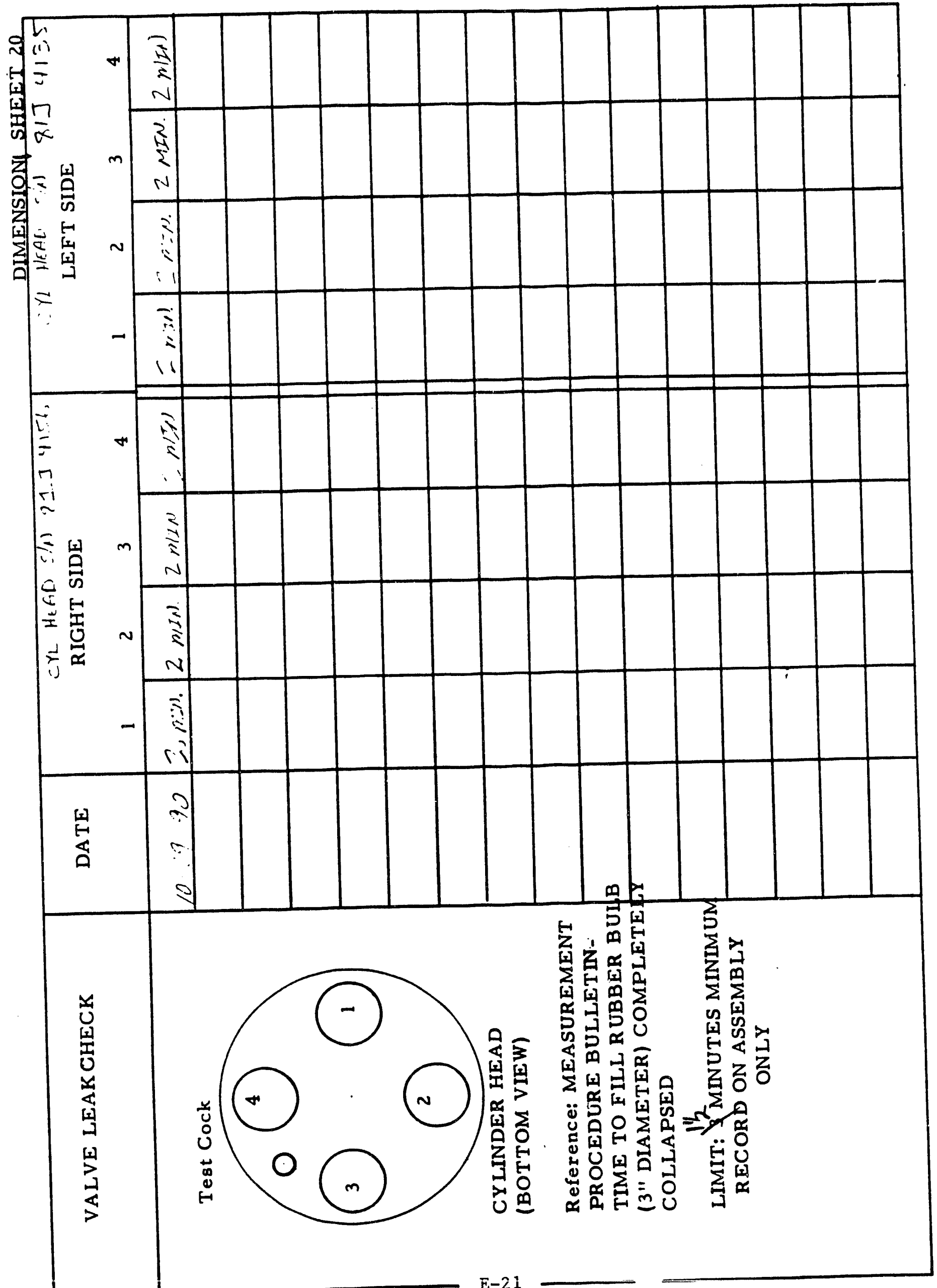




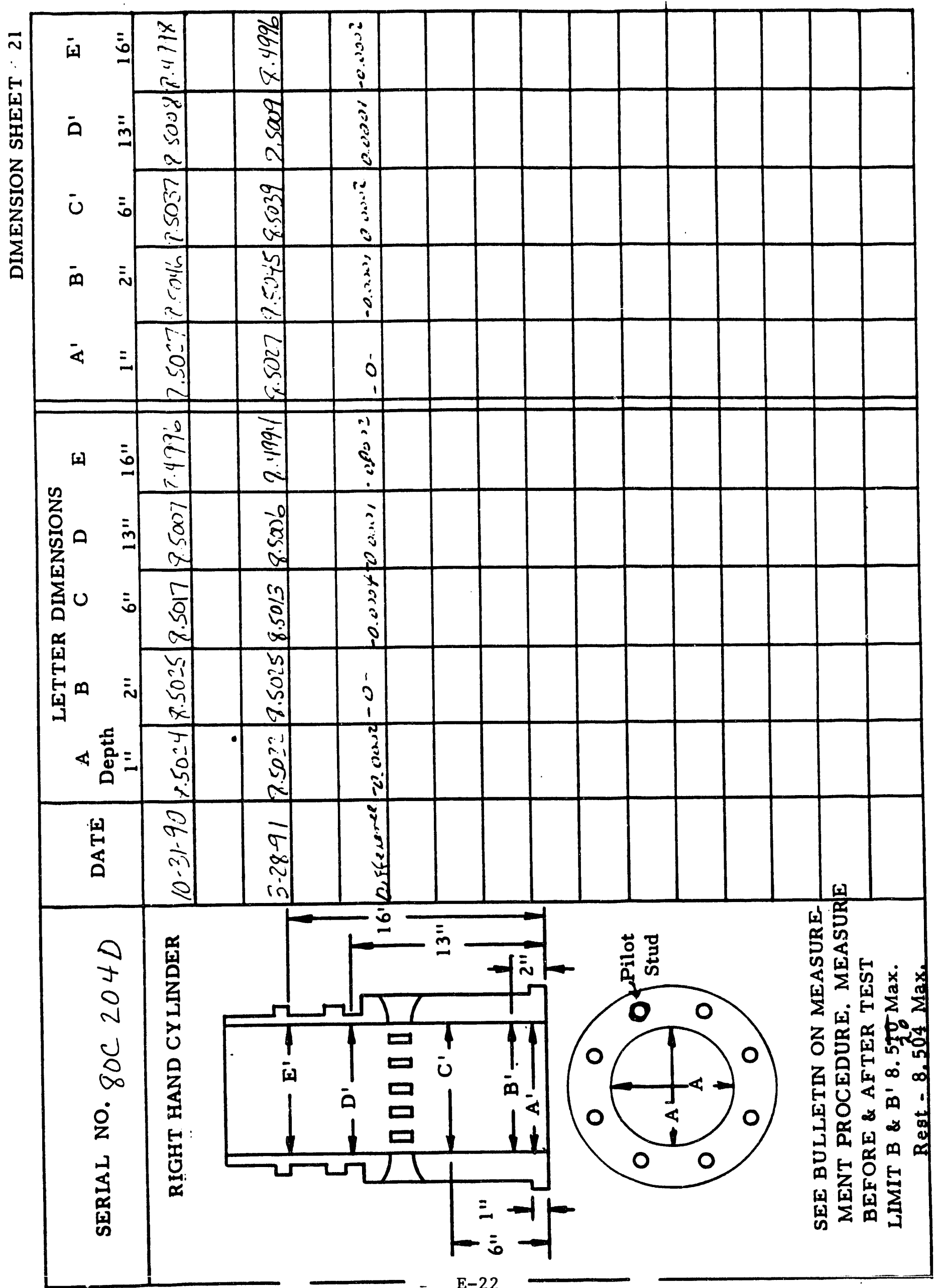




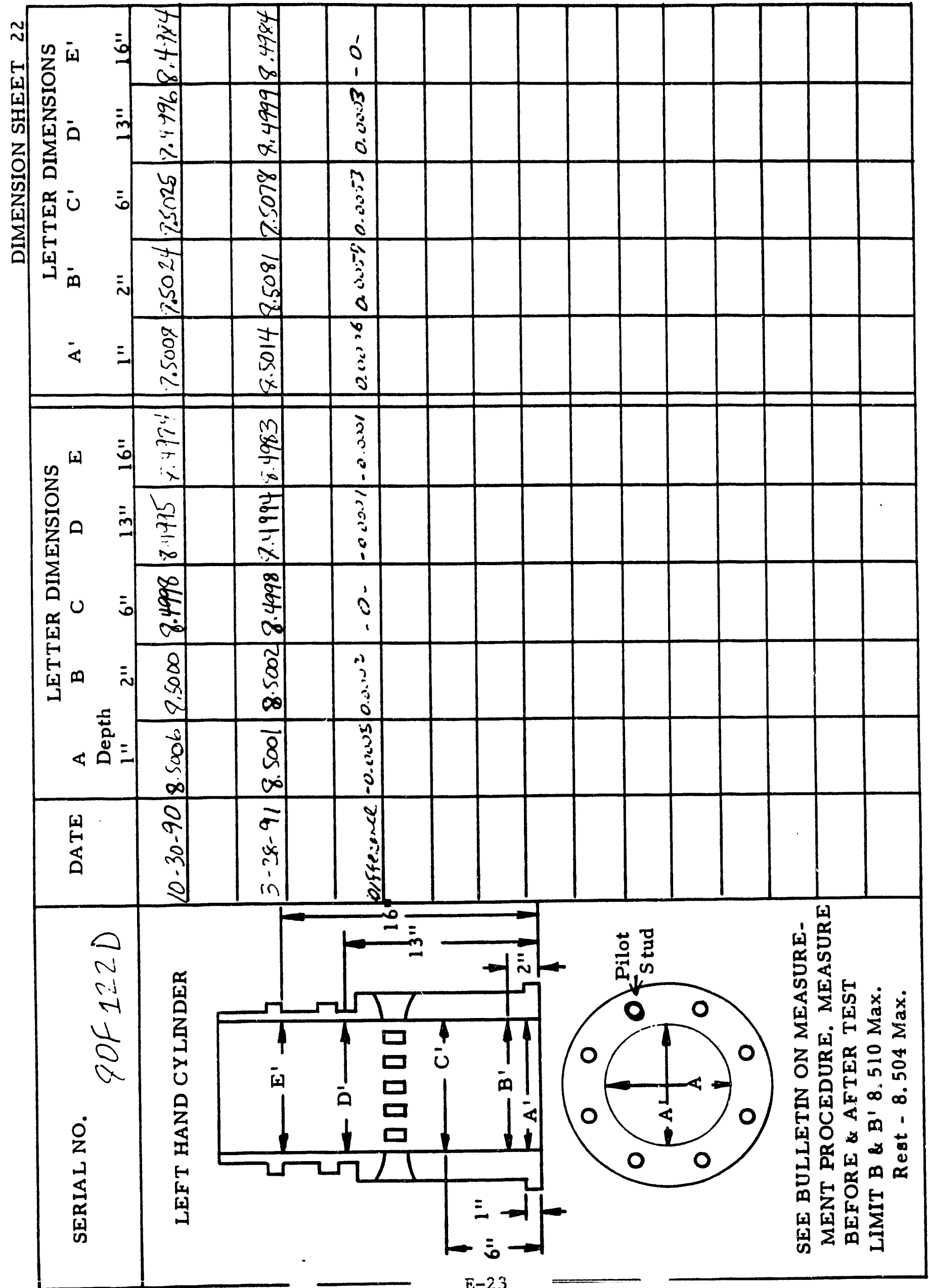




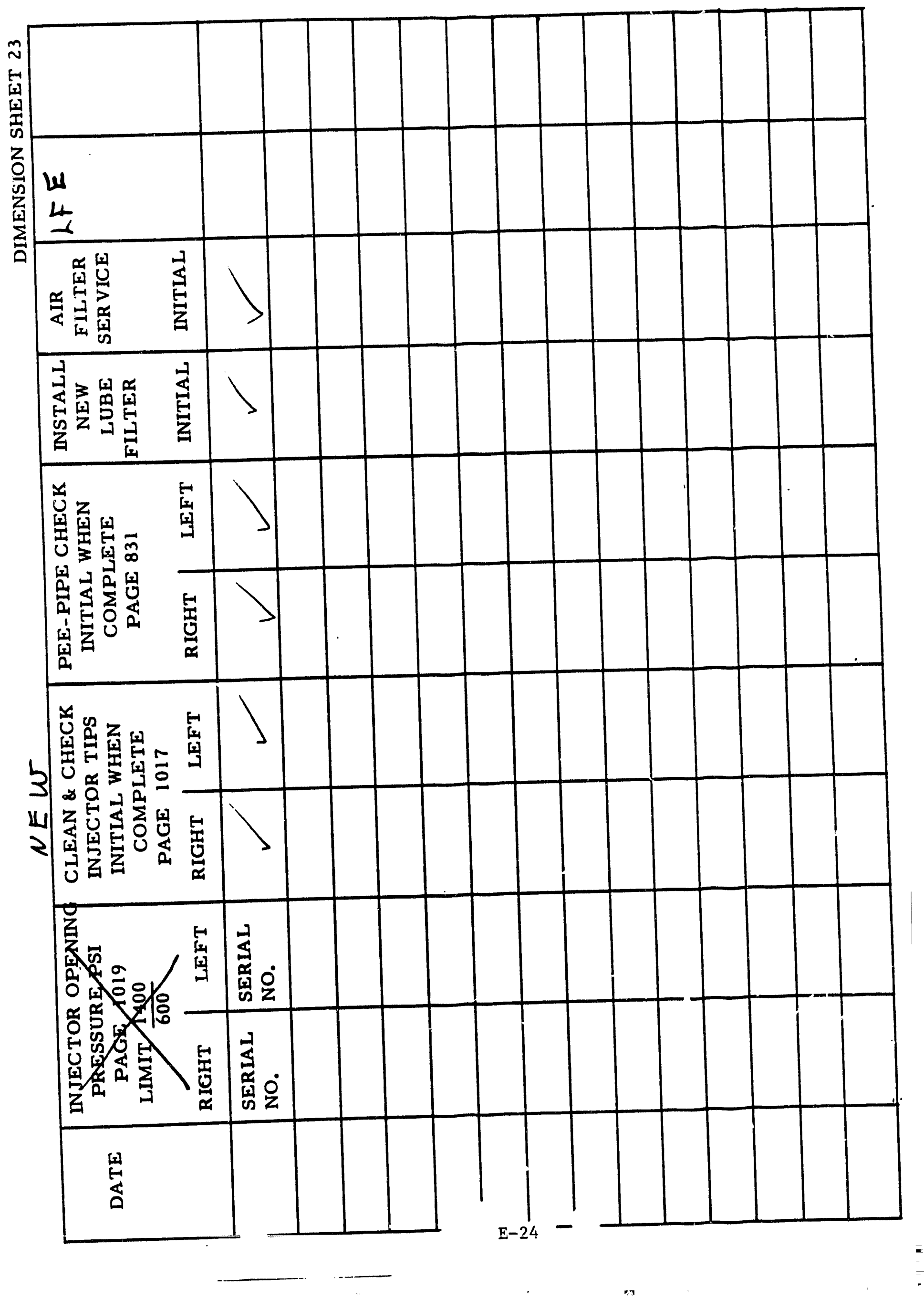




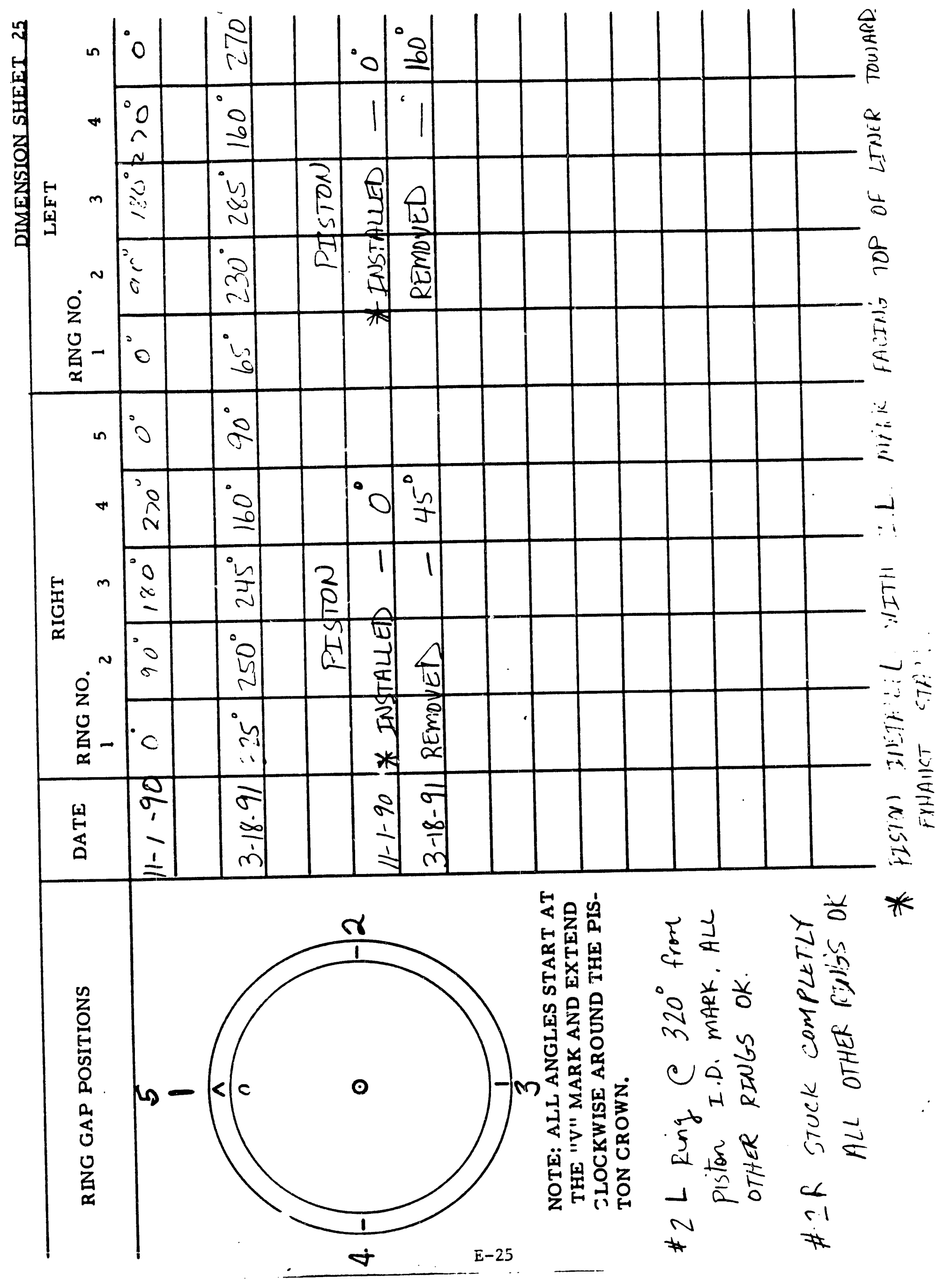


EMD TEST NO.

TEST PART WEIGHTS, GRAMS

Camshaft Bearings

\begin{tabular}{|c|l|l|l|l|l|}
\hline & Date & Right Front & Left Front & Right Rear & Left Rear \\
\hline 0 hours & & & & & \\
\hline 300 hours & & & & & \\
\hline
\end{tabular}

Pin Bearings

\begin{tabular}{|c|c|c|c|}
\hline & Date & Right & Left \\
\hline $0-$ hours & $11-14-90$ & 508.40 & 509.65 \\
\hline 300 hours & $3-19-91$ & 507.47 & 508.60 \\
\hline
\end{tabular}

Thrust Washers

\begin{tabular}{|r|c|c|c|}
\hline & Date & Right & Left \\
\hline 0 hours & $11-14-90$ & 215.62 & 715.33 \\
\hline 300 hours & $3-19.91$ & 214.12 & 712.82 \\
\hline
\end{tabular}

TEST PART DIMENSIONS, INCHES

Piston Pin Diameter (Parallel to Threaded Holes)

\begin{tabular}{|c|c|c|c|c|c|}
\hline \multicolumn{2}{|c|}{} & Date & Pee Hole End & Center & Opposite End \\
\hline \multirow{3}{*}{ Right } & 0 -hours & $11-14-90$ & 3.6845 & 3.6845 & $3.68+5$ \\
\hline \multirow{4}{*}{ Left } & 500 hours & $3-19-91$ & 3.6850 & 3.6849 & 3.6849 \\
\cline { 2 - 7 } & $0-$ hours & $11-14-90$ & 3.6440 & 3.6840 & 3.6839 \\
\cline { 2 - 7 } & 500 hours & $3-19-91$ & 3.6843 & 3.6943 & 3.6 .43 \\
\hline
\end{tabular}

Pin Bearing Clearance in Carrier

\begin{tabular}{|c|c|c|c|c|c|}
\hline \multicolumn{2}{|c|}{} & Date & Pee Hole End & Center & Opposite End \\
\hline \multirow{3}{*}{ Right } & 0 hours & $11-14-90$ & .010 & .013 & .016 \\
\cline { 2 - 7 } & 500 hours & $3-19-91$ & .009 & .013 & .016 \\
\hline \multirow{3}{*}{ Left } & 0 -hours & $11-14-90$ & .009 & .010 & .013 \\
\cline { 2 - 7 } & 000 hours & $3-19-91$ & .007 & .009 & .013 \\
\hline
\end{tabular}


APPENDIX F

F-1 

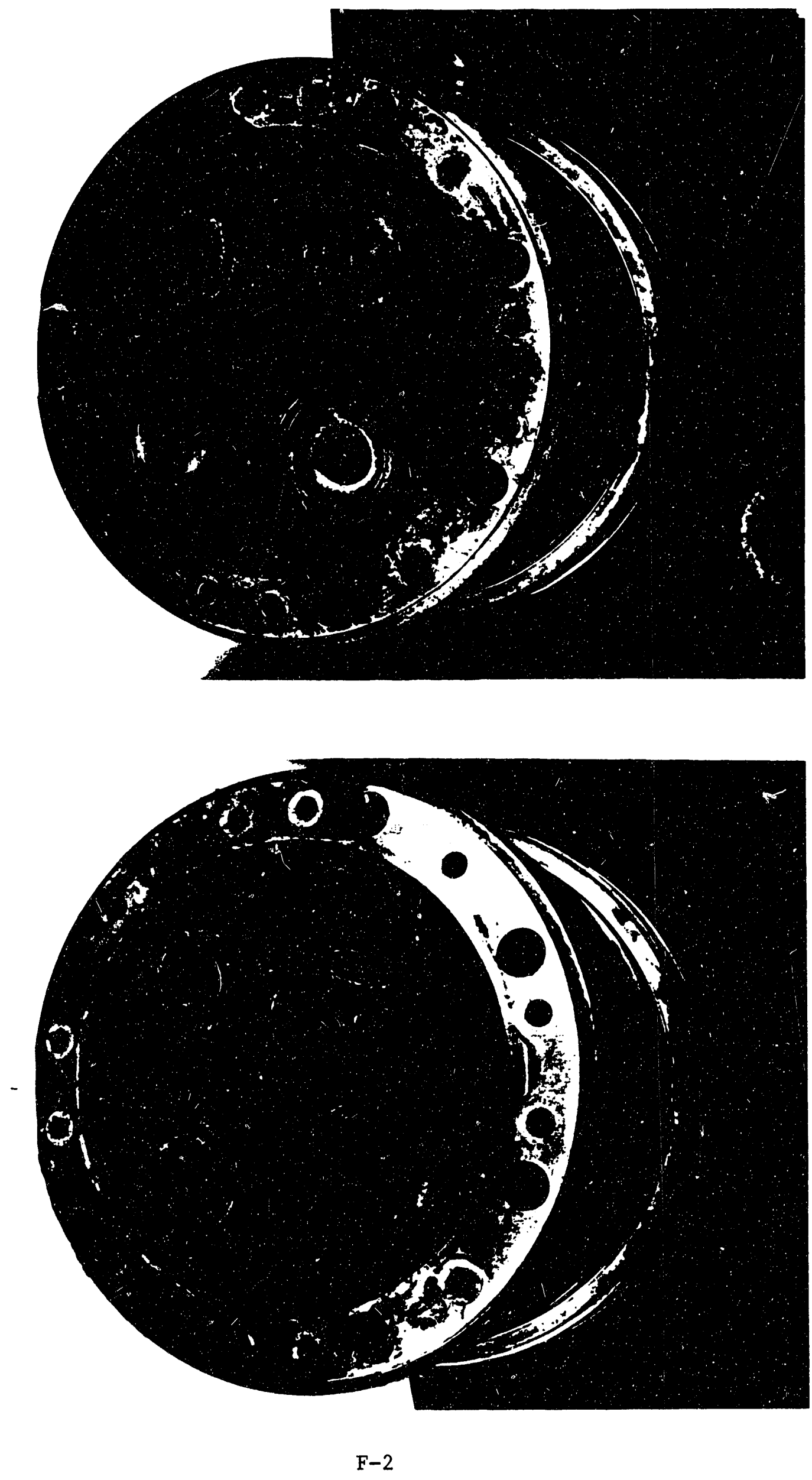

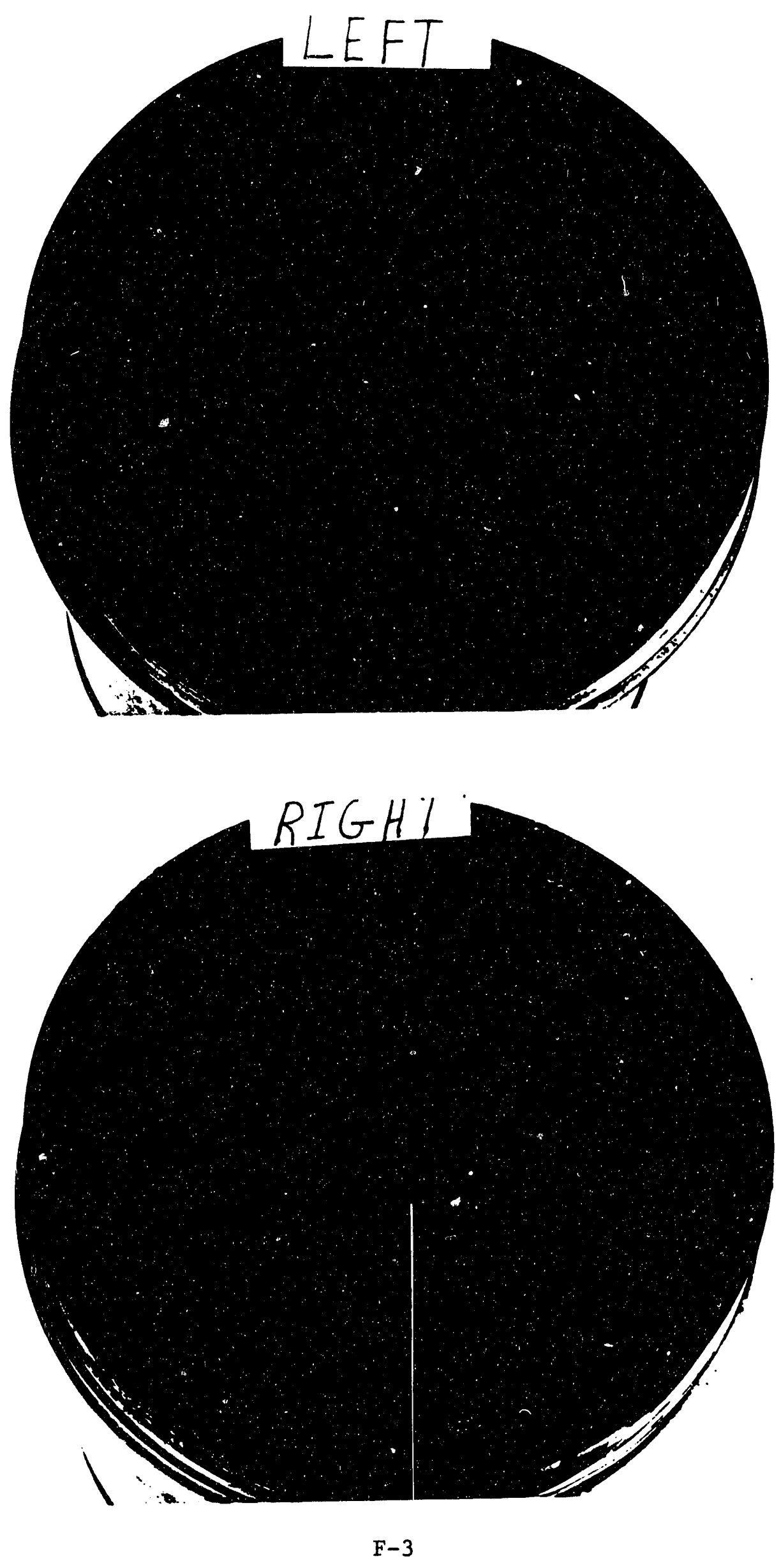

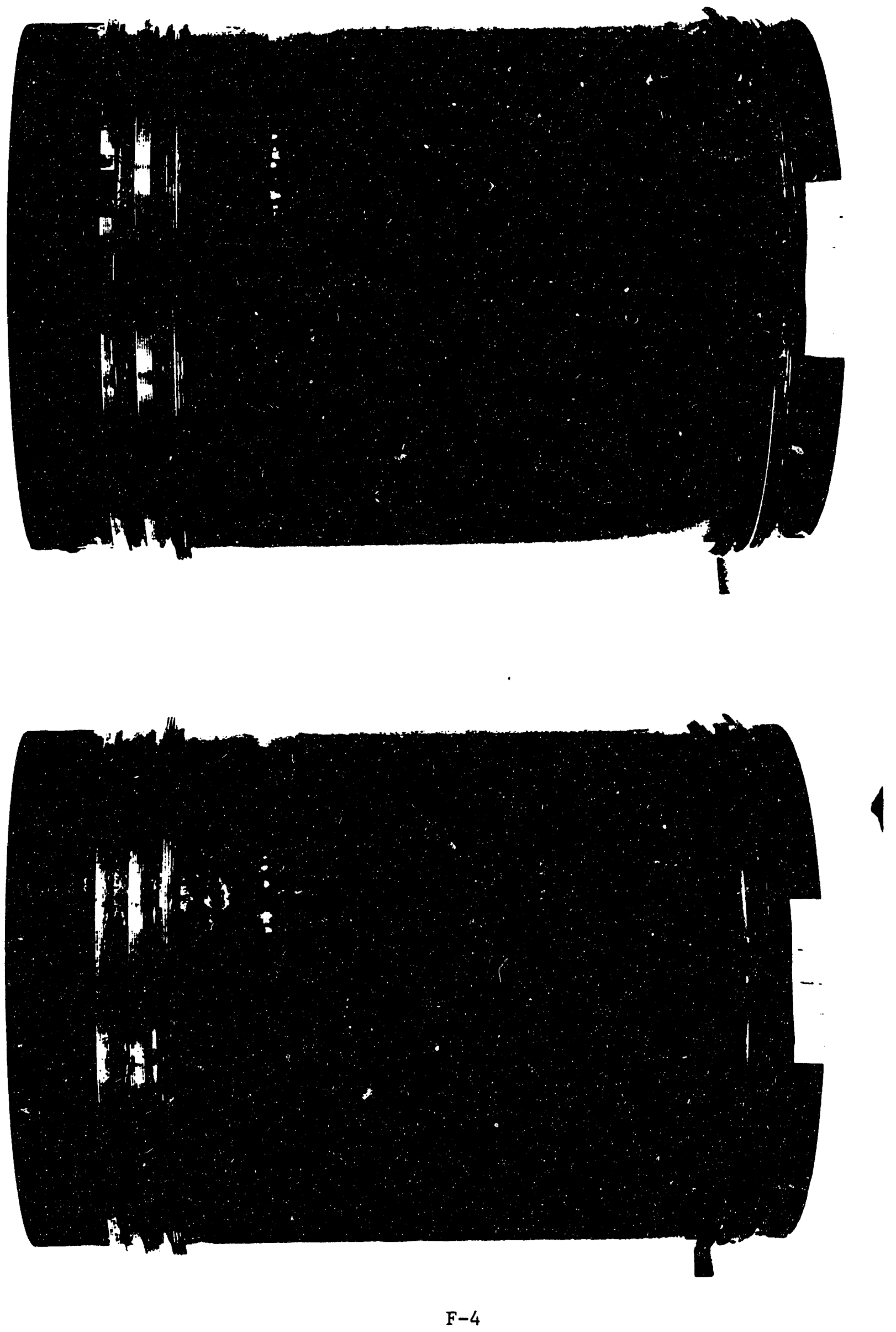


\section{LEFT}
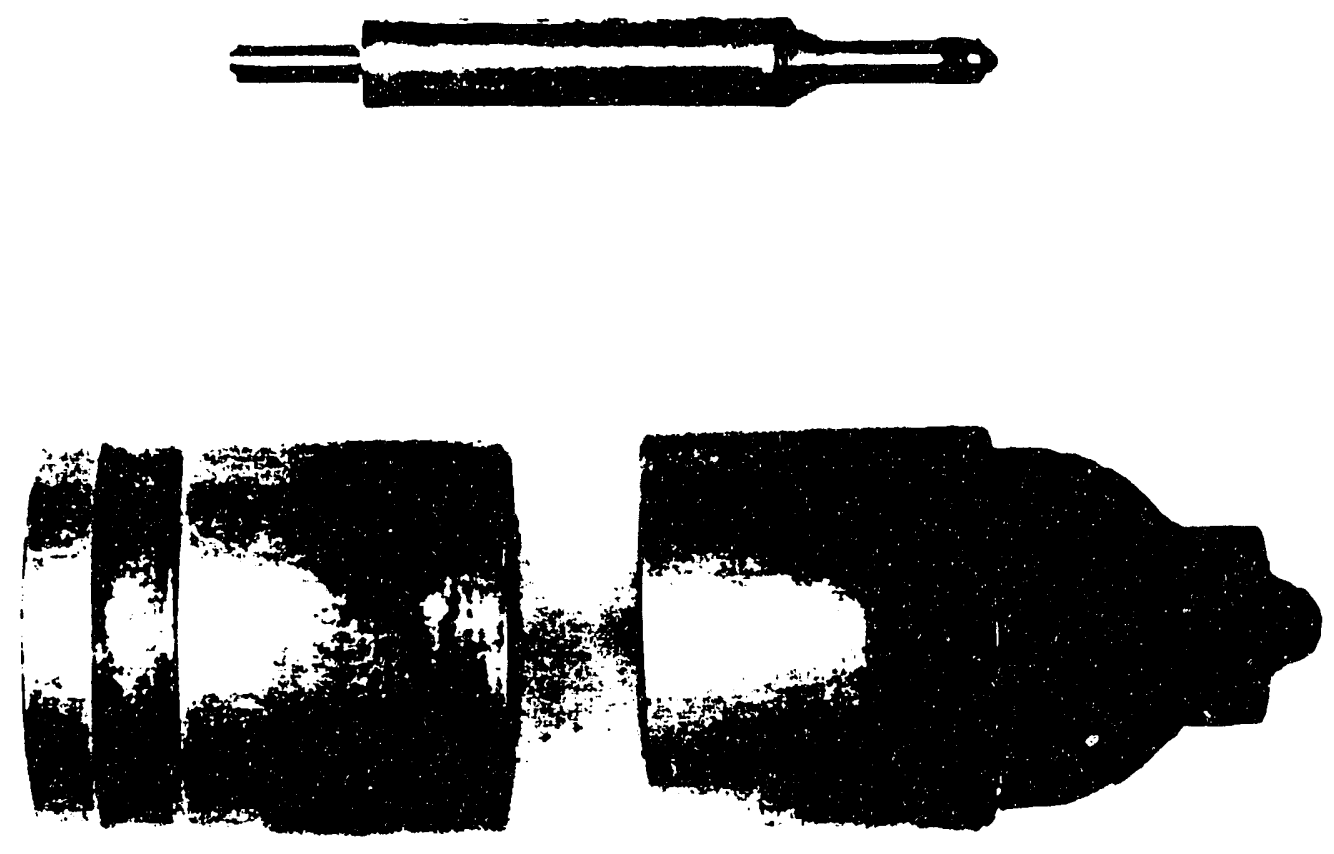

\section{RIGHT}
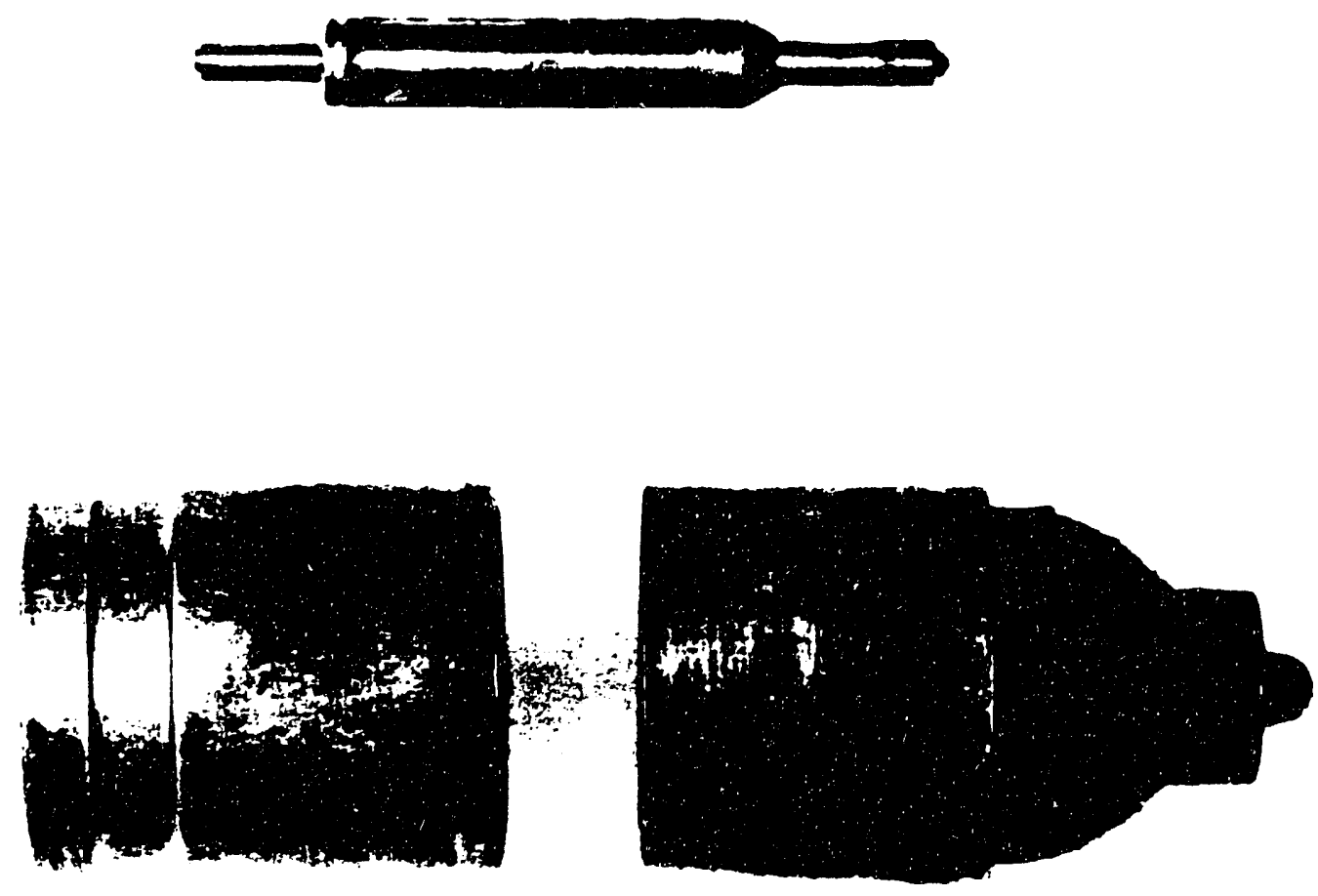

F-5 

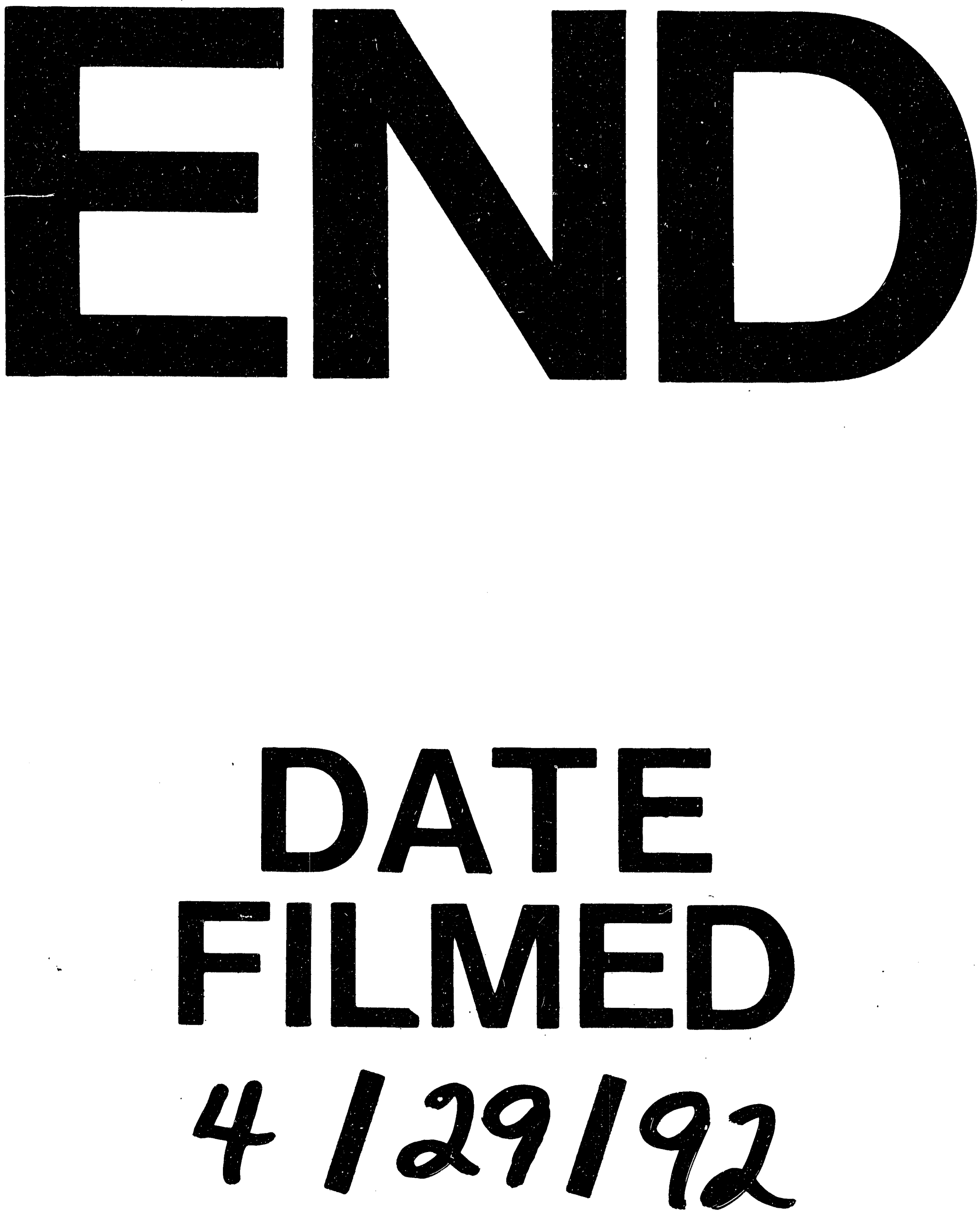
